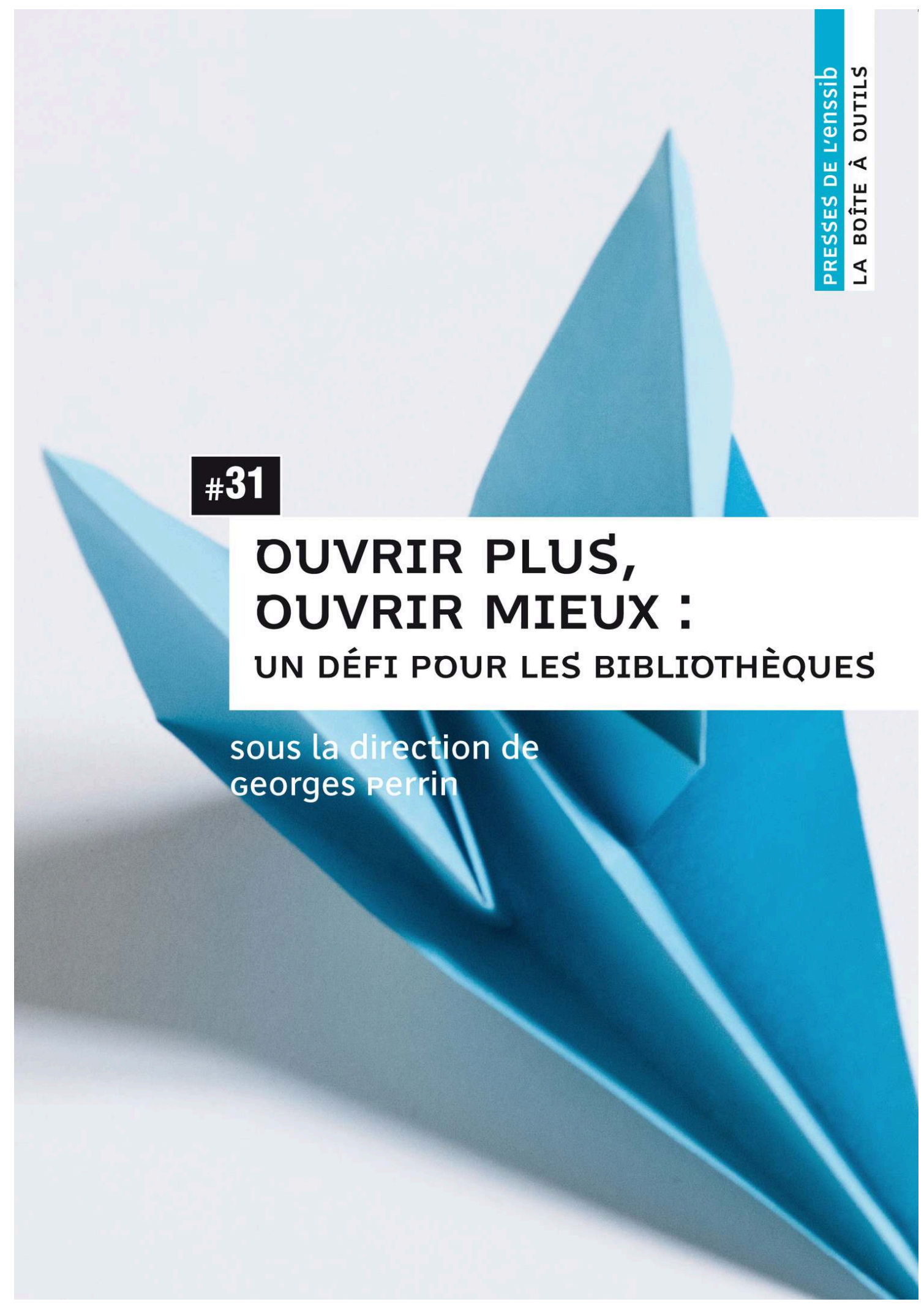




\section{Ouvrir plus, ouvrir mieux}

Un défi pour les bibliothèques

\section{Georges Perrin (dir.)}

DOI : 10.4000/books.pressesenssib. 3128

Éditeur: Presses de l'enssib

Lieu d'édition : Villeurbanne

Année d'édition : 2014

Date de mise en ligne : 10 décembre 2018

Collection : La Boîte à outils

ISBN électronique : 9782375460634

\section{Qboooks}

http://books.openedition.org

\section{Édition imprimée}

Date de publication : 1 janvier 2014

ISBN : 9791091281393

Nombre de pages : 176

\section{Référence électronique}

PERRIN, Georges (dir.). Ouvrir plus, ouvrir mieux : Un défi pour les bibliothèques. Nouvelle édition [en ligne]. Villeurbanne : Presses de l'enssib, 2014 (généré le 23 février 2021). Disponible sur Internet: <http://books.openedition.org/pressesenssib/3128>. ISBN : 9782375460634. DOI : https://doi.org/ 10.4000/books.pressesenssib.3128

(C) Presses de l'enssib, 2014

Conditions d'utilisation

http://www.openedition.org/6540 


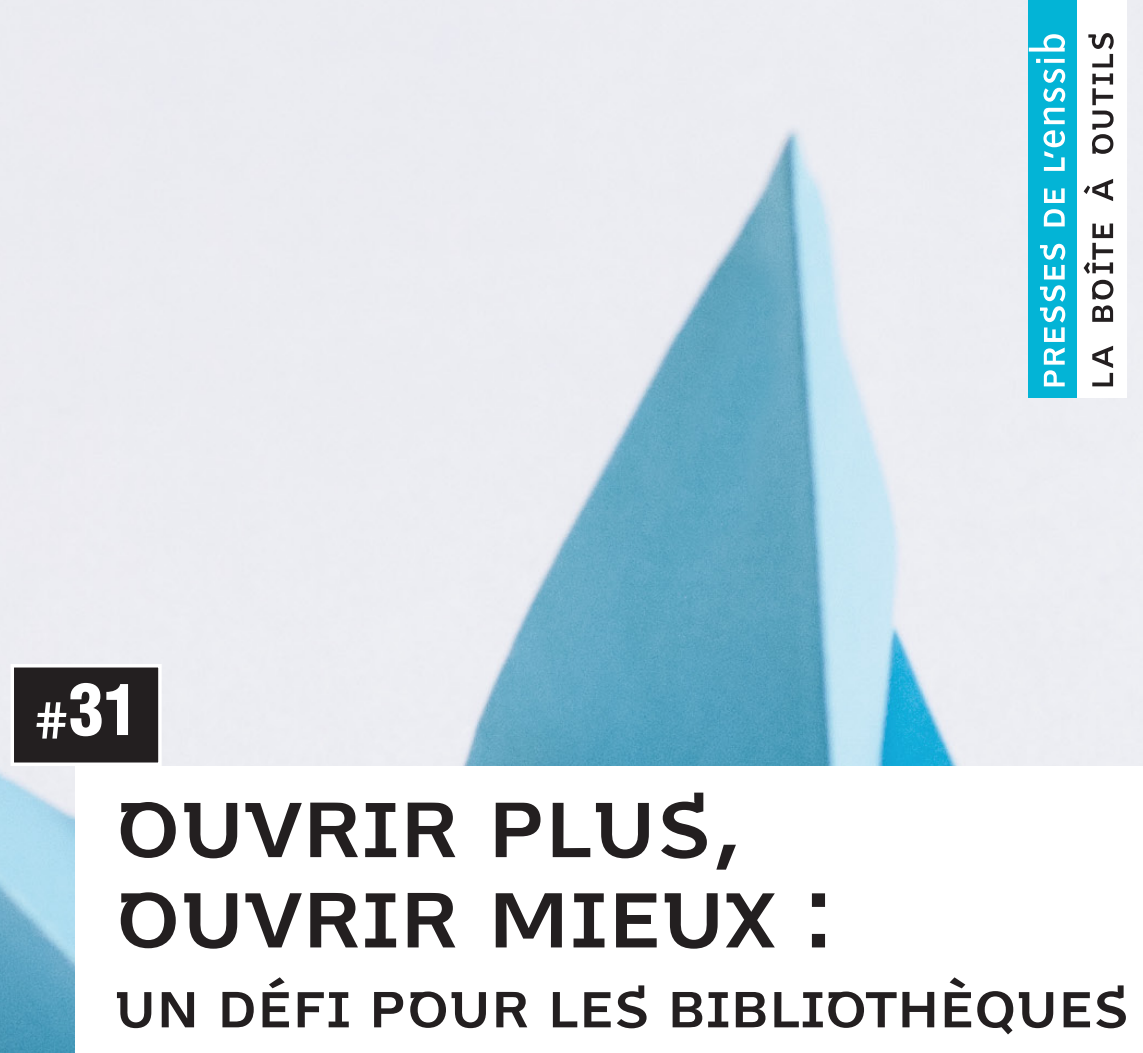

sous la direction de georges perrih 


\section{BAO\#31}

\section{OUVRIR PLUS, OUVRIR MIEUX: UN DÉFI POUR LES BIBLIOTHĖQUES}

Ouvrir, oui mais quand ? Matin, midi et soir ? Le dimanche ? Comment élargir les horaires d'ouverture, quels sont les obstacles à franchir ? Comment construire un tel projet, le porter et le mettre en œuvre ?

La France dispose aujourd'hui d'un des réseaux de bibliothèques les plus développés d'Europe. Toutefois, depuis quelques années, la stagnation du nombre de lecteurs inscrits oblige à une réflexion sur son évolution.

Parmi les causes de cette progressive désaffection figure la question des horaires d'ouverture qui, paradoxalement, n'a pas accompagné cette modernisation qui suppose un accueil élargi à tous les publics. La résolution de ce paradoxe appelle une réflexion sur le rôle social de la bibliothèque, aussi important que son rôle éducatif et culturel.

Prendre en compte les nouveaux modes de vie du public, resituer la vraie place de la bibliothèque dans la ville ou sur le campus, harmoniser l'utilisation de l'espace par les usagers avec leur disponibilité dans le temps, répondre concrètement aux nouvelles attentes des différents publics, renouveler les pratiques professionnelles en direction du public, tels sont les thèmes évoqués dans cet ouvrage.

Coordonné par Georges Perrin, inspecteur général honoraire des bibliothèques, ce volume réunit quinze professionnels spécialisés dans les domaines éducatif, social et culturel et témoigne d'autant d'expériences conduites avec succès pour une extension significative des horaires d'ouverture, et la très réelle amélioration de l'accueil qui s'en est suivi.

Mots clés : accueils, besoins des usagers, horaires d'ouverture, instituts français, pratiques professionnelles, représentations des bibliothèques, services aux usagers 
Ont contribué à cet ouvrage:

Chrystelle Amblard

Pierre-Yves Cachard

Jean Chaguiboff

Héloïse Courty

Pierre Franqueville

Hélène Grognet

Charlotte Henard

Jean-François Jacques
Monique Joly

Emmanuelle Paulet-Grandguillot

Georges Perrin

Claude Poissenot

Ophélie Ramonatxo

Isabelle Vazard

Isabelle Westeel
回常要品 Un bonus numérique Ouvrir différemment : un panorama, par Charlotte Henard, Georges Perrin et Isabelle Vazard, est à lire en ligne ou à télécharger sur le site de l'enssib : < http://www.enssib.fr/presses/ catalogue/ouvrir-plus-ouvrir-mieux >

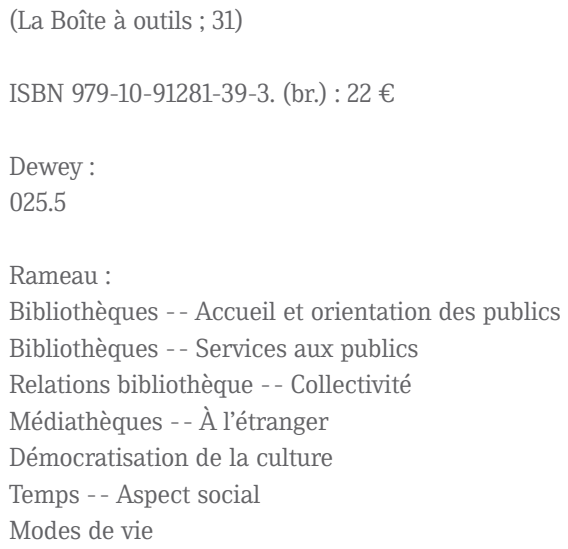

Notice rédigée par la bibliothèque de l'enssib. 


\section{MODE D'EMPLOI}

par Georges Perrin

\section{OUVRIR PLUS LES BIBLIOTHĖQUES : UNE PROBLÉMATIQUE COMMUNE, DES RÉPONSES DIVERSES ET ADAPTÉES}

Grâce aux efforts conjoints des décideurs au sein des collectivités territoriales et des universités d'une part, et des professionnels des bibliothèques d'autre part, les bibliothèques sont aujourd'hui en mesure de proposer à leur public dans toutes ses composantes une très grande variété de services sur place et à distance. Pour n'en citer que quelques-uns, rappelons qu'à la richesse et à la variété des collections sous toutes formes de supports, s'ajoutent désormais la qualité de l'accueil dans des locaux confortables, l'accès direct en ligne à toutes sortes d'informations, la proposition largement renouvelée d'actions culturelles diverses, la mise à disposition de locaux adaptés à la formation individuelle ou en groupe, l'accueil de services spécialisés dans la recherche d'emploi, etc.

Ce très important effort d'attractivité, rendu possible par l'accroissement considérable des surfaces et la modernisation des outils d'accès à l'information, a produit ses meilleurs effets sous la forme d'une diversification des publics, de leurs pratiques et de leur comportement. Il faut désormais ajouter au public des lecteurs dûment inscrits pour emprunter des documents, celui de ceux qu'on a appelés « les séjourneurs »*1, qui ne fréquentent les bibliothèques qu'en raison de la possibilité d'accès immédiat à la ressource documentaire et/ou culturelle, et du confort qu'elles offrent, source d'une convivialité sereine propice à l'appréhension de tous les domaines de l'actualité courante et à l'enrichissement culturel personnel. Toutefois, il manque à ce tableau presque idyllique un ultime élément qui touche à l'amélioration de la facilité d'accès à tous les avantages décrits plus haut : celui d'une amplitude des horaires d'ouverture* plus grande, plus complète et plus pertinente que celle d'aujourd'hui.

1. Les termes suivis d'un astérisque (à leur première occurrence) sont définis dans le glossaire en fin d'ouvrage. 


\section{GESTION DU TEMPS SOCIAL ET AMÉNAGEMENT DES TEMPS URBAINS}

Cette problématique a surgi dans le monde des bibliothèques depuis une dizaine d'années. Elle fait suite à l'évaluation de l'important effort qui a permis, au cours de ces trente dernières années, de couvrir l'ensemble du territoire d'un réseau de bibliothèques de qualité, et à l'inquiétude des professionnels constatant la baisse du nombre des usagers inscrits. Aussi, certaines réflexions de bibliothécaires et quelques études conduites par des sociologues ${ }^{2}$ ont permis de prendre conscience que sous l'effet de phénomènes nouveaux (entre autres, le développement du numérique et la modification progressive des modes et des rythmes de vie), le rapport des usagers à leur bibliothèque est en pleine mutation. Ces derniers sont désormais autant sinon plus sensibles à l'attractivité du lieu en tant que tel qu'à sa finalité première. Et, comme à l'égard d'autres services publics, ils sont désormais soucieux d'inscrire leur fréquentation de la bibliothèque dans un rapport qui leur est personnel entre le temps du travail, celui de la famille et celui des loisirs.

Il convient de noter que ce questionnement ne s'applique pas uniquement à la fréquentation des bibliothèques. Cette problématique rejoint en effet l'actualité des questions que pose aujourd'hui la pertinence des temps d'accès aux services en général (commerces, transports, loisirs, etc.). Comme la presse s'en est largement fait l'écho ces derniers mois ${ }^{3}$, les responsables des bibliothèques ne sont pas les seuls à se poser la question des ouvertures prolongées en soirée ou le dimanche. Tout ceci s'inscrit dans un panorama de questions de gestion du temps social auxquelles les responsables des collectivités locales de plusieurs pays d'Europe réfléchissent depuis une trentaine d'années.

Ce mouvement de réflexion, né dans quelques villes d'Italie dans les années 1980, a essaimé dans les pays voisins. En France, une structure

2. Voir entre autres : Bruno Maresca, Christophe Evans, Françoise Gaudet (collab.), Les bibliothèques municipales en France après le tournant d'internet : attractivité, fréquentation et devenir, Paris, Bibliothèque publique d'information, 2007 (coll. Études et recherche).

3. Pétition initiée en janvier 2014 par Bibliothèques sans frontières : "Ouvrons + les bibliothèques ! "; voir aussi : Véronique Heurtematte, " Ouvrir même le dimanche », Livres hebdo hors-série, août 2014 (Congrès IFLA). 
réunissant plusieurs villes a vu le jour en 2004, pour fédérer la réflexion sur l'aménagement des rythmes urbains et le phasage de l'ouverture des services publics avec la disponibilité de leurs usagers ${ }^{4}$. C'est dans un tel cadre, beaucoup plus large que celui de la bibliothèque, que se développe la réflexion des décideurs politiques sur les horaires d'accès aux services dont ils ont la charge.

De même, moins directement mais tout aussi sûrement, cette problématique en rejoint une autre, elle aussi, très actuelle. En effet, la nécessité d'un recours à un appoint complémentaire d'emplois à temps partiel pour des ouvertures plus longues s'articule complètement avec le souci de la puissance publique de favoriser l'insertion professionnelle des jeunes et notamment des étudiants.

D'autres questions d'ordre éducatif (réforme des rythmes scolaires) ou social (aide à la recherche d'emploi) qui font notre actualité, peuvent être recoupées par celle d'un élargissement du temps d'accès aux bibliothèques. Certaines municipalités l'ont d'ores et déjà compris. D'autres restent plus hésitantes. Dans d'autre cas, enfin, l'accord entre professionnels et décideurs sur ce sujet n’a pas encore trouvé de forme décisive.

\section{PRÉSENTATION DE L'OUVRAGE}

Comme nous le verrons plus loin, si la très grande majorité des professionnels a pris conscience de cette problématique, toutes les bibliothèques n'ont pas encore mis en œuvre des actions nécessaires à un élargissement significatif de leurs horaires d'ouverture. En effet, une telle mise en œuvre s'avère complexe : pour n'évoquer que deux des nombreux préalables à cet élargissement des plages d'ouverture, citons la réflexion sur le renouvellement de l'organisation du travail interne et la difficulté d'évaluer les moyens complémentaires (personnels et budget) qu'il convient de déployer. Mais beaucoup d'autres questions se posent auxquelles chaque

4. « Tempo territorial », dont les statuts ont été déposés en 2004, et qui fédèrent aujourd'hui 27 collectivités territoriales (conseils régionaux, conseils généraux, communautés urbaines, communautés d'agglomérations, villes) et 2 universités. Voir la contribution de Chrystelle Amblard : " Le temps de la ville : l'évolution des rythmes de vie interpelle l'offre de services publics », p. 62. 
bibliothèque doit répondre en fonction de sa spécificité et de son environnement local.

Aussi, dans ses différentes parties, cet ouvrage s'efforce de décrire, à partir d'exemples divers, les réflexions et les actions préparatoires à la mise en œuvre couronnée de succès de cet élargissement des ouvertures en soirée, le dimanche ou périodiquement, en raison de circonstances particulières.

La première partie de l'ouvrage permet de faire un point sur l'état d'avancement de ces mises en œuvre dans l'ensemble du paysage des bibliothèques françaises. Elle fait état de réflexions générales sur l'intérêt d'accueillir un public renouvelé grâce à des plages de temps améliorées, et ce, quelle que soit la dimension de la bibliothèque : premier service culturel d'une petite ville ou centre du réseau documentaire d'une grande université. L'ensemble de ces réflexions et de ces expériences montre bien que la bonification des services offerts par chaque bibliothèque est très corrélée à l'amplitude de son ouverture.

Dans la deuxième partie, sont exposées les conditions préalables à la réussite d'une extension des horaires d'ouverture. Avoir une connaissance la plus précise possible de son public et de ses attentes, prendre en compte le temps nécessaire aux étudiants pour utiliser au mieux et selon leur rythme propre, ce lieu de vie qu'est la bibliothèque universitaire $(\mathrm{BU})^{5}$, inscrire la durée d'ouverture de la bibliothèque dans la temporalité de la cité, programmer les espaces au moment de la conception de la future bibliothèque de telle sorte qu'ils puissent accueillir confortablement celles et ceux qui souhaitent rompre momentanément avec le rythme effréné de l'environnement urbain, créer le bon niveau de relations entre les décideurs politiques et les professionnels des bibliothèques pour se donner les meilleures chances de succès lors de la mise en œuvre d'une ouverture élargie de la bibliothèque, tels sont les différents aspects de cette problématique évoqués dans cette partie. Et cette présentation des actions mises en œuvre s'accompagne de l'examen de tous les aspects pratiques, toutes les conséquences les plus concrètes sur la vie des agents et toutes les

5. Pour les sigles et acronymes, se reporter à la liste en fin d'ouvrage. 
incidences financières pour la collectivité. Il s'agit, en effet, de réussir la phase préparatoire à cette opération.

L'ensemble de cette revue ne serait pas complet sans une troisième partie exposant des expériences réussies, qu'il s'agisse d'ouvertures le dimanche ou de très larges ouvertures en continu incluant des "nocturnes » consistantes. C'est en suivant les étapes de leur préparation, de leur mise en œuvre et des évaluations et bilans successifs qui ont permis de les améliorer au fil du temps, qu'on peut avoir des indications plus précises pour bâtir un projet d'élargissement des horaires d'ouverture. Dans la mesure où ils contreviennent à certaines idées reçues sur le sujet, où ils montrent tous les bénéfices qui découlent de ce type d'action, tant pour les usagers que pour les personnels, les témoignages exposés dans ce cadre sont de nature à rassurer les professionnels qui veulent affronter ce défi et à les conforter dans leur volonté de réussir l'amélioration de l'accueil de leurs publics. Dans cette partie est également exposé l'intérêt partagé entre les agents titulaires de la bibliothèque et les personnels d'appoint que constituent les étudiants, pour des ouvertures élargies.

Enfin, l'exposé illustratif de la variété des pratiques développées permettra d'esquisser des pistes susceptibles de conduire au plein succès des opérations d'extension des horaires d'ouverture, pour parfaire enfin l'image des bibliothèques et leur permettre de préserver pour longtemps leur rang d'établissements culturels les plus fréquentés de notre pays.

LIBRE ACCÈS $\rightarrow$ Ouvrir différemment : un panorama, par Charlotte Henard, Georges Perrin et Isabelle Vazard, est à lire en ligne ou à télécharger sur le site de l'enssib : < http://www.enssib.fr/presses/catalogue/ouvrir-plus-ouvrirmieux > 


\section{PARTIE I}

\section{L'OUVERTURE ÉLARGIE DES BIBLIOTHĖQUES : WORK IN PROGRESS}

1. CONDÉ-SUR-NOIREAU, UNE BIBLIOTHĖQUE TRÈS OUVERTE par Isabelle Vazard

$++++++++++++++++++++++++++++++++++++++++++++++++++++++++++++++++t$

2. LES ÉTUDIANTS EN BU : DE NOUVEAUX COMPORTEMENTS ET DE NOUVELLES ATTENTES QUI NOUS OBLIGENT par Pierre-Yves Cachard

+ +++++++++++++++++++++++++++++++++++++++++++++++++++++++++++++++

3. UNE BIBLIOTHĖQUE OUVERTE SUR DES INDIVIDUS EN CONSTRUCTION par Claude Poissenot

4. OUVRIR OPPORTUNÉMENT : LA MÉDIATHĖQUE DU KREMLIN-BICÊTRE par Héloïse Courty

5. DE LA BIBLIOTHĖQUe AU LEARNING CENTER (LILLE 3) : UN ACCUEIL RENOUVELÉ POUR TOUS LES PUBLICS

par Isabelle Westeel 


\section{1}

\section{CONDÉ-SUR-NOIREAU, UNE BIBLIOTHĖQUE TRĖS OUVERTE}

par

Isabelle Vazard

La médiathèque de Condé-sur-Noireau (Calvados, 5300 habitants) a ouvert en 1990 avec 36 heures d'ouverture hebdomadaire. J'ignorais à l'époque que cette large amplitude horaire, tout à fait atypique, allait déterminer ce qu'est devenu maintenant " l'Atelier » : un lieu bien identifié, au cœur de la ville, ancré dans la vie locale. L’Atelier est effectivement devenu le lieu de ressources incontournable de la ville incluant la médiathèque, l'espace musée, l'office de tourisme et le Point info 14 (service d'accompagnement social, relais de différents partenaires dont Pôle emploi, Caisse d'allocations familiales, Caisse primaire d'assurance maladie, etc.). Il accueille aujourd'hui près de 100000 visiteurs par an.

II a fallu penser le mode de fonctionnement approprié dès la création de la médiathèque. La question était la suivante : de façon concrète, opérationnelle et acceptable pour le personnel, comment offrir un service public culturel de qualité ? Le pragmatisme s'est révélé comme la seule option viable : il fallait faire table rase de nos pratiques anciennes, en inventer de nouvelles et si possible, de meilleures. La bonne nouvelle, ça n'a pas été si compliqué.

La volonté politique forte affirmée par les élus nous a facilité la tâche : il fallait faire avec ce que l'on avait et établir des priorités réalistes et bien définies. Pour nous, ce fut l'accueil avant et au-dessus de tout. Quand les priorités sont claires, le reste peut se négocier au fur et à mesure. Nous avons donc pris la décision assez radicale de ne pas disposer de services internes, nos banques de prêt et d'information faisant ainsi office de bureaux. La polyvalence s'est également imposée, nous n'étions pas assez nombreux (4 emplois temps plein) pour fonctionner autrement.

\section{OUVRIR, C'EST SE POSER LA QUESTION DU PUBLIC}

La question du choix des heures d'ouverture amène inéluctablement à s'interroger sur son environnement : comment vit la population qui nous 
entoure et à quel rythme ? Comment réellement faciliter l'accès à tous les publics ? Il nous était clair qu’à Condé-sur-Noireau, la population vit au rythme des commerces. La vie, hors du travail, se tient là et il faut donc s'inscrire dans cette démarche : aller à la médiathèque comme on fait ses courses. On comprend facilement que ces horaires doivent être lisibles et compréhensibles par tous si on veut effectivement attirer ce large public. Nous avons donc strictement calqué nos horaires sur ceux des commerces. L'ouverture du dimanche n'était donc pas primordiale. Cependant, en 2007, avec la création de l'espace musée au sein de la médiathèque, la question de l'ouverture dominicale s'est posée à nouveau.

Cette décision concernant la grande amplitude horaire d'ouverture était fortement liée à l'importance accordée au public et à notre préoccupation première de favoriser l'accès aux différents espaces et aux ressources mises à disposition. Il s'agissait également de créer un lieu de vie, favorisant les occasions de rencontres et d'échanges. On le sait, les usagers familiarisés avec les lieux culturels tels que les bibliothèques ne s'embarrassent pas de la question des lieux, des espaces, voire même de l'accueil et des horaires d'ouverture. Ils sont coutumiers de leurs modes de fonctionnement et ils ont intégré dans leur emploi du temps les heures d'ouverture, connaissent les rayonnages qui leur sont chers ainsi que les modalités de prêt.

Mais, l'espace d'une seconde, déplaçons notre point de vue de professionnel et considérons objectivement l'ensemble des contraintes liées à la fréquentation d'une bibliothèque par un non-initié : ai-je vraiment le droit d'entrer dans ce lieu ? Dois-je payer à l'entrée ? Vais-je comprendre son fonctionnement ? Quelqu'un va-t-il m'aider ? C'est au regard de ces questions qu'il faut comprendre la décision d'adosser la fréquentation de la bibliothèque à d'autres pratiques et particulièrement celles liées à la fréquentation des commerces.

La situation géographique, en centre-ville, combinée aux heures d'ouverture importantes a contribué au succès immédiat de la médiathèque. Le bâtiment est idéalement situé car intégré à un itinéraire emprunté communément par les habitants. La fréquentation de la médiathèque ne nécessite donc aucune démarche spécifique et préméditée. Nous avons accueilli, dès l'ouverture, un public nombreux de non habitués qui nous a, en quelque sorte, "éduqués » à d’autres pratiques professionnelles. 


\section{UN PERSONNEL AU SERVICE DU PUBLIC}

\section{PRIORITÉ AU FRONT OFFICE*}

C'est entendu, il n'y aurait pas de bureau. Il est vrai que cette décision a entraîné quelques aménagements en ce qui concerne l'organisation physique des postes de prêt et également au niveau du fonctionnement au quotidien, aux yeux et à l'écoute de tous. La priorité étant donnée au service au public, le reste des tâches s'opère en période creuse. Cette flexibilité dans l'organisation du travail est comprise et appréciée par le personnel. De plus, en accordant au public la possibilité de venir à la médiathèque au moment qui lui convient, la circulation du public est plus fluide. On évite les files d'attente aux différents postes et le personnel est plus disponible pour renseigner et accompagner les usagers de la médiathèque. Ce fonctionnement exclusif en front office, qui semble encore peu courant, représente pourtant une des solutions simples pour accroître les heures d'ouverture.

\section{Assumer et organiser}

Il est néanmoins indispensable que cette priorité accordée au public soit clairement établie et que les conséquences engendrées par ce fonctionnement soient pleinement assumées par la hiérarchie. On doit accepter que les rayonnages ne soient pas à tout moment scrupuleusement rangés, que l'équipement des documents ne soit pas immédiat. Il faut combiner des objectifs clairement déterminés à une organisation à la fois précise et flexible. Pour ce faire, chaque mardi, avant l'ouverture, nous disposons de 30 minutes pour planifier en équipe la semaine à venir. Il s'agit de planning, d'organisation et peu de contenus, seuls les projets à venir sont évoqués rapidement. C'est le seul moment de la semaine où l'équipe se réunit au complet, tous services confondus. Ce temps, extrêmement court, est crucial pour informer les uns et les autres des différentes activités proposées au sein de l'Atelier. Il permet aussi de rappeler à tous les priorités, les décisions concernant le fonctionnement : il est essentiel que lors de cette réunion, la même parole soit entendue par tous. La mise en place 
de nouveaux projets fait ensuite l'objet de micro-réunions, sur le temps d'ouverture, avec les deux ou trois personnes concernées.

\section{Polyvalence et compétences}

La démarche qualité*, initiée en 2005 par l'ensemble des services de la commune a structuré l'organisation interne de la médiathèque. Elle a permis de déterminer et de dégager deux groupes de compétences : les compétences de base et les compétences spécifiques. Les premières relèvent directement de la polyvalence du personnel et permettent à chacun d'être en mesure d'occuper l'ensemble des postes de prêt et d'accueil. Les compétences spécifiques sont plus complexes à définir car elles sont le fruit de différents facteurs : aptitudes personnelles, savoir-faire antérieurs, connaissances professionnelles, niveau d'études sans oublier la culture acquise dans le cadre de son parcours personnel. Même si ces compétences spécifiques ne sont pas forcément identifiées dès l'arrivée dans l'équipe, il est important de se donner le temps de les repérer. C'est souvent la valorisation de ces compétences personnelles qui est source d'épanouissement et qui donne un sens à l'implication au quotidien. Depuis 20 ans, le personnel s'est renouvelé : l'équipe a accueilli des compétences nouvelles, elle a rajeuni, l'équilibre homme/femme est davantage respecté. Ce sont des facteurs non négligeables quand il s'agit d'accroître les heures d'ouverture. En 2007 puis en 2011, l'intégration de nouveaux services agissant sur le champ culturel mais aussi social et touristique a posé une nouvelle fois la question de la polyvalence du personnel. En mutualisant ces compétences, l'objectif principal consistait à répondre dans un même lieu à des attentes culturelles, à des problématiques sociales mais également à amplifier la visibilité de chaque service en faisant se rencontrer ces différents publics. Nommée directrice de l'Atelier, mon rôle a davantage consisté à valoriser la complémentarité des compétences dans un même établissement qu'à imposer une polyvalence des personnels. En fonctionnant sur les mêmes créneaux horaires dans un même lieu, il m'est apparu essentiel de favoriser le partage de l'information, de faire en sorte que chaque service ait une connaissance précise du champ d'action des autres services et de faire travailler les équipes autour de projets communs. En mai dernier, les personnels de la médiathèque et du Point Info 14 ont 
animé conjointement des ateliers en direction du public scolaire dans le cadre de la "Semaine de l'Europe ». À l'occasion du 70e anniversaire du débarquement, en juin 2014, des visites de la ville et l'organisation du salon du livre ont été préparées par le personnel de la médiathèque, du musée et de l'office de tourisme.

\section{Décloisonner les espaces intérieurs}

Une des clés de la réussite de la médiathèque réside dans l'aménagement de l'espace : des plateaux décloisonnés, des perspectives dégagées permettant d'un seul coup d'œil d'envisager l'ensemble. L'Atelier se veut avant tout un lieu collectivement partagé, les rayonnages contribuant seuls à créer des espaces plus intimes.

Il nous a paru essentiel de penser l'espace intérieur en prenant en compte le regard du non habitué et, tout particulièrement, dès l'entrée. On sait que ce premier regard est essentiel. On sait aussi que les habitués des bibliothèques savent qu'ils vont y trouver au minimum des livres. Alors pourquoi installer des rayonnages dès l'entrée ? J'imagine même qu'à Condé-sur-Noireau, certains usagers ont oublié qu'ils se trouvaient dans une bibliothèque : en effet, on découvre d'abord le kiosque, la cafétéria, l'espace multimédia ainsi que l'espace permettant l'accès à l'espace musée et cela sans aucun rayonnage en vue.

L'aménagement intérieur est en quelque sorte l'illustration de notre fonctionnement. Le décloisonnement des espaces permet au personnel d'être réactif, d'agir avec une grande souplesse et favorise l'accompagnement du public. Les connections Internet se situent à proximité du poste de prêt audiovisuel. On encourage par ce fait la demande d'aide, qui est, par expérience, assez fréquente autour des postes informatiques. Cette configuration des lieux, très " ouverte ", favorise la visibilité du personnel et de surcroît sa mobilité. On est à la fois en mesure de conseiller un lecteur tout en ayant un regard sur son poste de prêt. Ce mode de fonctionnement peut paraître exigeant pour le personnel, car pour le coup, il se trouve très sollicité. Il est surtout très gratifiant. Chacun récolte personnellement le fruit de son implication. C'est un véritable moteur qui ne doit pas être sous-estimé. 


\section{L'ATELIER, UN PROCESSUS}

Dans les faits, l'Atelier est devenu un établissement regroupant une médiathèque, un musée, un service social lié aux problématiques de l'emploi et un office de tourisme. Dans la réalité, c'est un lieu de vie, d'échanges qui se questionne et qui évolue constamment. Cette dynamique est avant tout le résultat d'une proximité étroite entretenue avec le public depuis plus de 20 ans combiné à un " état d'esprit » du personnel qui y travaille : chacun dans son domaine a conscience de participer à un processus de valorisation profitable à tous. L'Atelier rend des micro-services à toutes sortes de personnes, est reconnu en tant que tel et le personnel contribue

pleinement à ce " cercle vertueux ». La commune en a fait sa vitrine en y organisant de nombreuses manifestations, souvent hors du champ culturel. En 2007, le projet de création d'un musée, dans les cartons depuis deux mandats, voit le jour dès l'instant où la proposition de l'associer à la médiathèque est avancée.

\section{L'ESPACE MUSÉE CHARLES LÉANDRE, LA MUTUALISATION EN MARCHE}

L'idée d'adosser le futur musée à la médiathèque a émergé de façon très logique : il s'agissait de mettre en commun les moyens, notamment ceux liés au personnel et de travailler à la cohérence de l'offre culturelle à l'échelle de la ville. La dénomination " espace musée ", en tant qu'espace de la médiathèque, sa gratuité et l'adoption d'horaires communs ont posé de façon claire la relation étroite souhaitée entre les deux structures et affirmé la volonté de créer une dynamique entre les deux établissements. Deux agents, chargés de l'animation du musée ont été recrutés et ont intégré le schéma existant en matière de polyvalence. Le travail en commun des deux équipes s'est concrétisé rapidement autour du programme d'animations, les expositions temporaires sont présentées en partie au sein de la médiathèque pour renforcer cette synergie. La mise en commun profite aux deux espaces : les usagers de la médiathèque ont pris l'habitude de visiter l'espace musée lorsqu'ils viennent emprunter des documents et le 
public plus éloigné géographiquement, habitué des musées, découvre et apprécie la singularité de ce lieu aux propositions multiples.

\section{L'ATELIER, ESPACE DE COHÉSION SOCIALE}

La décision d'intégrer un service social, étroitement lié à l'emploi, dans un établissement culturel répondait à une question que tous les professionnels des bibliothèques se posent : comment faire venir à la médiathèque ces personnes qui, quoi que l'on propose, ne viennent pas spontanément? Les animations, les visites de scolaires, la diversification des supports ont un impact limité sur ce public. La difficulté réside essentiellement dans notre incapacité à établir un contact avec ce public. Le passage obligé par la médiathèque du public du Point info 14 nous a permis de créer les conditions favorables à l'appropriation des différentes propositions par ce nouveau public. Nous avons donc multiplié les passerelles entre les deux services : des ressources numériques en autoformation* ont été mises en place dans les deux structures. Deux fois par mois, dans l'auditorium de la médiathèque, un partenaire du Point Info 14 vient à la rencontre du public. Le forum de l'emploi, qui a connu cette année sa $8^{\mathrm{e}}$ édition, rassemble 800 visiteurs dans la médiathèque. C'est aussi l'occasion, pour la trentaine de partenaires, acteurs locaux de l'emploi et de l'insertion de se rencontrer. Le personnel des deux structures a dû s'apprivoiser, apprendre à travailler ensemble. Des ajustements ont été nécessaires mais on en mesure au quotidien les bienfaits. Des gens qui n'auraient jamais dû se croiser se rencontrent, des initiatives prennent forme grâce à ces rencontres. Pour preuve, la création d'un fonds documentaire autour de l'emploi et de la formation est actuellement en projet.

\section{UN OFFICE DE TOURISME, ET PUIS QUOI ENCORE ?}

Déjà fortement impliqué dans la promotion de l'établissement, l'office de tourisme a trouvé sa place tout naturellement à l'Atelier. La mise en commun des compétences s'est concentrée sur l'apport de propositions dans notre programmation culturelle, notamment autour de la mise en valeur du patrimoine et de la mémoire locale. La proximité des services facilite le partage de l'information. Le réseau de partenaires de l'un profite à 
l'autre, des liens se tissent et la promotion de l'Atelier s'en trouve amplifiée. Les publics se croisent : c'est utile pour un touriste en recherche d'informations de trouver dans le même lieu un office de tourisme, un musée à visiter et le wifi. L'usager de la médiathèque peut venir y chercher ses places de spectacle, le programme de cinéma et préparer ses prochaines vacances.

\section{Partenariats et convergence d'initiatives : l'aide aux devoirs}

Dans une petite ville de 5000 habitants, on repère facilement les bons relais, les énergies positives. On prend vite conscience que certains veulent donner et que d'autres sont en demande. Les faire se rencontrer n'est pas inscrit dans nos missions mais c'est à notre portée. Le dispositif " aide aux devoirs ", mis en place il y a 7 ans, est né de ce constat. Depuis, nous accueillons à la médiathèque chaque soir quinze enfants en difficulté scolaire, identifiés par les enseignants. Une vingtaine de bénévoles motivés, d'horizons divers, portent ce dispositif : on commence par goûter et après on travaille. Une charte est signée en début d'année qui engage l'enfant, les parents et le bénévole. Ces bénévoles constituent un relais considérable qui participe activement à la promotion de l'établissement. En tissant des liens, en faisant se rencontrer les acteurs institutionnels et associatifs, l'Atelier est maintenant devenu un lieu de convergence d'initiatives.

\section{La bibliothèque départementale de prêt (BDP), facilitateur de projets}

En reconnaissant notre capacité à valoriser des services innovants, la BDP du Calvados est devenue un allié essentiel de l'évolution de la structure. Ces dernières années, son soutien financier et son expertise nous ont permis de développer avec succès un secteur jeux vidéo ainsi que la « Boîte numérique », plate-forme de ressources en ligne en autoformation, soutien scolaire et cinéma. De plus, en proposant un catalogue de formations pertinent, elle accompagne de façon opérationnelle le questionnement sur l'évolution de notre métier. 


\section{L'avenir de l'Atelier : de nouveaux défis à relever}

Le fonctionnement de l'Atelier interroge nos missions. Nous faisons chaque jour le grand écart entre jeux vidéo et recherche généalogique, actualisation au Pôle emploi et catalogage, programmation d'expositions et autoformation en Français langue étrangère. C'est quelquefois déstabilisant mais c'est passionnant. Ce n'est pas un modèle, juste une possibilité. L'Atelier aujourd'hui, c'est $33 \%$ d'inscrits, 100000 visiteurs par an, et pas de fermeture annuelle : derrière ces chiffres, une réalité faite de remises en question, d'ajustements et surtout de «petites victoires ». Ce qui est sûr, c'est que de nouveaux défis nous attendent... alors, restons ouverts ! 


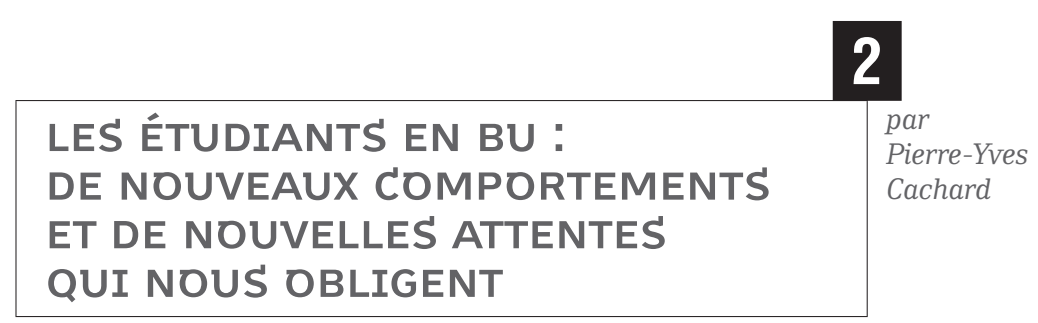

On s'intéresse encore trop peu aux effets des ruptures générationnelles sur la nature et la forme des services offerts au sein des bibliothèques. Cette question paraît pourtant d'autant plus légitime dans les bibliothèques de l'enseignement supérieur que le renouvellement de ce public s'opère systématiquement par classe d'âge.

On attribuera donc l'extension des horaires d'ouverture des services documentaires au sein de nos établissements à une politique d'incitation menée par le ministère de l'Enseignement supérieur et de la Recherche et à des initiatives courageuses (ou masochistes selon que l'on aime ou pas les négociations tendues) locales, portées par une direction ou une équipe, dans un contexte favorable ou impérieux : l'ouverture d'un nouvel équipement, la pression d'un lobby disciplinaire ou l'opportunité d'un projet d'établissement.

L'ouverture étendue s'inscrirait ainsi dans la droite ligne des politiques de modernisation, comme le libre accès par exemple. Il faut cependant noter que le libre accès précisément a été encouragé dans un contexte de massification des effectifs étudiants, et donc déjà à partir d'un constat d'évolution démographique.

D'innombrables publications sont consacrées à l'étude des difficultés d'insertion des nouvelles générations dans le monde du travail, en relation avec les changements de comportement observés et largement attribués aux pratiques massives des nouvelles technologies. La question que l'on devrait se poser est de savoir s'il est nécessaire d'ouvrir plus et mieux, sous la pression des politiques d'indicateurs nationales et locales ou en conséquence logique des changements observés tant dans la perception de la bibliothèque par ses lecteurs étudiants que dans les nouveaux usages apparus au sein de nos espaces? 
Cette question est importante, car les freins subis en interne et les réticences souvent exprimées lors des négociations entre les établissements mettant en place une démarche d'extension des horaires d'ouverture de la bibliothèque, et les professionnels chargés de l'appliquer, ont sans doute limité la portée des avancées réalisées, et en menacent même, dans une certaine mesure, la pérennité pour les solutions reposant sur des dispositifs temporaires ou offrant des conditions d'accueil dégradées par rapport aux périodes d'ouverture normale.

\section{DES POINTS DE VUE DIFFICILES À CONCILIER}

Pourquoi ce résultat ? Certes, parce que les moyens humains et financiers à disposition des établissements imposent des solutions économes et innovantes. Mais aussi parce que des deux côtés de la négociation, on n'examine pas le même objet. Pour les équipes de direction, il s'agit avant tout de répondre favorablement à une demande explicite du public, le plus souvent dans le cadre des enquêtes de satisfaction mises en œuvre ces dernières années, ou parfois dans un contexte plus houleux de revendications étudiantes. Pour la plupart des personnels des services concernés, il s'agit de fixer des limites et un cadre à la disponibilité professionnelle, évaluée au regard d'une vie sociale ou familiale extérieures au cadre professionnel. Aucun de ces deux points de vue n'est en réalité moins légitime que l'autre : ils ne parlent simplement pas du même endroit, et nécessitent le plus souvent d'opter pour une négociation de compromis et de mesures compensatoires. On a peu l'occasion d'observer sur ce sujet un consensus dans la méthode et la démarche entre les deux parties.

La vision des établissements se concentre sur les usages dans un contexte d'analyse des coûts complets, de dispositifs d'aide à la réussite des études universitaires, et avec une logique utilitariste d'optimisation des ressources documentaires et immobilières. Pour simplifier, plus d'heures d'ouverture entraîne dans le cadre d'une bibliothèque universitaire, à la fois plus de publics (parce que les rythmes présentiels sont plus soutenus dans certaines disciplines et composantes), et plus de temps de présence de l'étudiant. À l'inverse, des horaires inadaptés ou réduits mettent en cause le bien-fondé des investissements financiers réalisés pour ce service. Une 
lecture d'autant plus tentante qu'elle englobe aujourd'hui dans son champ de réflexion, la question délicate de la masse salariale et des effectifs professionnels. Les moyens affectés versus les usages constatés ou, dit autrement, le niveau d'efficience du service au sein de l'établissement et pour une communauté d'usagers définie.

Sans prétendre résoudre ici l'approche antagoniste, complexe et contrastée, il peut être utile de rappeler que l'enjeu des extensions d'horaires d'ouverture des bibliothèques concerne un métier dans son ensemble, et non une juxtaposition d'établissements. Cette attente des usagers pour une amplitude horaire étendue nous distingue, c'est une évidence, des autres services des établissements d'enseignement supérieur. Face à la généralisation progressive de ces démarches, qui concernent aujourd'hui tout autant les bibliothèques des collectivités territoriales que celles des universités, la lecture dramatique et conflictuelle de cette problématique semble avoir tendance à s'apaiser. La question est d'ailleurs désormais systématiquement prise en compte ou instruite dans la programmation architecturale et la préfiguration des nouveaux équipements.

Deux aspects manquent encore pour faciliter sinon l'adhésion, du moins une démarche de co-construction pendant la phase de négociation:

- la modulation des horaires de travail ou les mécanismes compensateurs (régime indemnitaire, récupération) devraient donner lieu à un accord de branche, au niveau de la filière. Il y a beaucoup à dire sur le manque de dispositions réglementaires aujourd'hui permettant une compensation attractive des contraintes d'organisation induites ${ }^{1}$. Cela favorise les solutions substitutives (monitorat étudiant, automatisation, fonctionnement dégradé, restrictions d'accès) qui ne constituent parfois que des réponses partielles aux attentes exprimées, et affaiblissent le potentiel d'accueil comme la légitimité des

1. Il faut rappeler qu'il est bien évidemment impossible d'opérer une extension d'horaires au sein d'un service d'accueil du public à dépense financière constante. 
compétences documentaires professionnelles. Là-dessus, du moins, on peut considérer qu'il y a un assez large consensus ;

- la prise en compte dans la réflexion des évolutions constatées dans les nouvelles classes d'âge étudiantes. C'est moins le «pour quoi » qui manque, parce qu'il est alimenté et documenté par un contexte accompagné par des discours et s'articule avec des problématiques fortes (réussite en licence, innovation pédagogique), que le "pour qui ». Il y a d'évidence une attente, et qui ne s'explique pas uniquement par une impérieuse nécessité documentaire ou la recherche désespérée d'une place de travail. C'est sur ce second point que l'on va s'attarder ici.

\section{YZ, DE LA CONNECTIVITÉ INDIVIDUALISTE AU NOMADISME COMMUNAUTAIRE}

Le concept de génération fait référence à des tranches d'âges pour lesquelles on considère que le fait de partager un même contexte (événements, environnement social, politique, culturel, technologique) induit des comportements et des attentes très similaires. La particularité des universités, c'est qu'elles concentrent en leur sein, et pendant plusieurs années une population d'usagers qui n'est quasiment constituée que d'individus d’une même génération.

Ce concept doit être apprécié à deux niveaux :

- les caractéristiques communes propres à une génération, qui peuvent faciliter la caractérisation du besoin et des attentes dans le cadre des projets de modernisation de nos services ;

- les clivages, "frictions », ou les crispations qui naissent de la cohabitation parfois difficile entre différentes générations partageant le même espace. Ceux qui définissent les services offerts en bibliothèque, appartiennent pour la plupart à des générations antérieures. Il n'est pas surprenant que l'on retrouve 
au final dans nos services le même type de problématiques relationnelles, ou les mêmes inadéquations des méthodes et des principes appliqués aux générations actuelles que ce qui a pu être observé en milieu professionnel.

La plupart des auteurs qui ont théorisé ces effets générationnels, s'accordent pour distinguer 4 générations. Les deux premières ne concernent plus les étudiants (" baby-boomers, baby bust* ou génération $\mathrm{X}^{*}$ »), mais elles constituent encore une part importante de nos effectifs professionnels. Les deux suivantes sont celles sur lesquelles nous devons porter notre attention :

- la génération Y* (1980-1994), les digital natives, qui forment actuellement encore le gros des effectifs étudiants dans les établissements, et ont pour la plupart tourné le dos à la lecture papier au profit de la lecture écran. Les bibliothèques se sont senties un temps menacées dans leurs missions historiques par cette révolution des pratiques de lecture, qui ont paru s'accompagner dans certaines disciplines et certaines fractions de la communauté universitaire (les chercheurs) d'un abandon de la bibliothèque, lieu physique. Il faudra attendre le grand virage numérique des années 2000 pour que les bibliothèques soient en capacité d'afficher une offre de service en adéquation, sur la partie documentaire, avec les aspirations de cette génération et adaptée à sa culture ;

- la génération Z* (1995-...), qui commence tout juste à intégrer l'enseignement supérieur. Elle est tout aussi digital native que la précédente, mais s'en distingue - et c'est important - par la nature des usages qu'elle fait des technologies. C'est une génération hyper connectée, parfois dénommée génération $C$, pour Communication, Connexion, Création. C'est une génération qui s'est emparée du Web social, et considère les réseaux comme un nouvel espace de sociabilité. Elle organise son 
quotidien dans un balancement permanent et complémentaire entre virtuel et réel.

\section{GÉNÉRATION Y : UNE APPROCHE RADICALEMENT DIFFÉRENTE DE LA CONNAISSANCE}

La génération $Y$ est souvent considérée comme particulièrement difficile à appréhender par la génération $X$ et les institutions. D'une part, parce qu'elle a tendance assez naturellement à mettre en cause les règles établies, et à adopter une certaine liberté de ton et d'action, et d'autre part, parce qu'elle se caractérise par un rapport différent à la culture et aux connaissances. Souvent déconsidérée voire dépréciée par les générations précédentes pour un manque apparent de culture et de curiosité, c'est une génération « du court terme et de l'immédiateté », qui considère que sa maîtrise des technologies lui permet à tout moment d'accéder rapidement et de mobiliser les connaissances nécessaires. La connaissance pour eux se mesure moins à la maîtrise des acquis qu'à celle des accès. Ces étudiants sont sensibles à la connectivité offerte, ils fonctionnent en tribus, mais restent individualistes, sont créatifs, adaptables et soucieux d'innover par rapport aux modèles qui leur sont imposés. Mais en bibliothèque, l'un des comportements qui les caractérise le mieux, c'est une grande capacité d'adaptation, un rejet de toute catégorisation et un fonctionnement multitâche qui a rapidement mis à mal les formations documentaires aux outils, et les stratégies de profilage des postes informatiques et les procédures complexes d'accès aux services. Une capacité multitâche qui interdit la hiérarchisation des activités : il n'y a plus de différence de nature entre Youtube, ScienceDirect, Google, Wikipedia et la plate-forme pédagogique. C'est ainsi qu'un des principaux cadres de référence de la profession est réduit à néant, ou produit un discours de filtrage des usages qui ne rencontre que peu de crédit du côté des étudiants concernés. Facteur aggravant, démontrant l'indéniable malignité de la génération $\mathrm{Y}$, on a pu constater une indifférence croissante aux agencements par supports, et aux topographies logiques et linéaires.

En milieu professionnel, l'approche managériale d'un individu de la génération Y évite prudemment la contrainte et privilégie le participatif. On ne devrait pas résister à un étudiant de la génération Y et lui fournir simplement et immédiatement ce qu'il demande, et sans même envisager de lui expliquer 
comment l'exploiter dans les règles de l'art. Avec un peu de chance, on sera étonné par ce qu'il parvient à produire avec. En bibliothèque, l'appréhension du phénomène a été sans doute lente, mais elle s'est traduite par une révolution dont nous ne mesurons pas encore aujourd'hui sans doute tous les effets : une modification de l'organisation globale des services, qui, au contact de ces usagers d'un " troisième type » est passée d'une conception orientée collections à une conception orientée publics. La satisfaction des étudiants de la génération Y imposait d'assouplir les codes, d'ouvrir le réseau, d'y transposer progressivement nos offres (services et collections) et de réduire les contraintes, parmi lesquelles les restrictions des horaires. Il n'est pas tout à fait certain que tout le monde ait compris au sein de nos services que ces bouleversements des habitudes des "baby-boomers » et des « baby bust » étaient moins dus à l'obstination opiniâtre des équipes de direction, qu'à la présence influente des étudiants de la génération Y.

\section{GÉNÉRATION Z : DE L'HYPER CONNEXION AU WEB SOCIAL}

La génération $\mathrm{Z}$ fait encore assez peu l'objet d'études en milieu professionnel car elle y est pour l'instant très peu représentée. C'est donc le système éducatif qui fournit aujourd'hui l'essentiel des portraits robots dont nous disposons. C'est une génération hyperconnectée, qui a grandi avec les réseaux sociaux inventés par la génération Y. L'organisation hiérarchique et verticale n'a plus de sens pour elle. La plupart des activités lui semblent naturellement transférables en ligne, et par ailleurs, elle ne conçoit pas sa connectivité sans technologies nomades. Les frontières entre l'espace domestique et l'espace public, entre la vie professionnelle et personnelle sont définitivement abolies. L'articulation entre les espaces physiques et virtuels est totale : les réseaux sociaux nourrissent et régissent son réseau de relations dans le quotidien, et elle en reproduit les codes ou les attentes dans la réalité. Dans ce contexte, la bibliothèque joue un rôle important d'espace de sociabilisation et d'échanges, plus que de documentation sans doute. Cette génération en attend confort, accès au réseau, sécurité et stabilité. Les notions d'heures de travail n'ont plus véritablement d'importance et échappent au principe de continuité. L'espace physique lui-même se nomadise, car le travail peut se faire n'importe où, mais l'étudiant y recherchera un environnement rassurant. 
Le travail collaboratif lui est plus naturel que le travail individuel en autonomie. Des collaborations qu'il ira chercher indifféremment au sein du Web social ou à la bibliothèque.

On pourra objecter que cette vision est réductrice et caricaturale, et qu'elle évacue tous les autres facteurs de différenciation, notamment sociaux, les nuances disciplinaires, culturelles, qui introduisent dans notre quotidien en bibliothèque de subtils dégradés réconfortants dans ces portraits générationnels dressés à gros traits. Mais il est impossible au quotidien pour nos établissements de prendre en compte toute l'étendue de la diversité des besoins qui peuvent s'exprimer au sein d'une classe d'âge. Pour faire évoluer nos services, nous nous appuyons le plus souvent de manière plus ou moins consciente sur les tendances lourdes observées dans nos espaces de lecture.

\section{UNE BIBLIOTHĖQUE YZ : VERS UN AUTRE ESPACE-TEMPS}

Qu'on les appelle Learning Centre* ou pas, les bibliothèques du futur sont bien sûr dessinées pour ces deux générations d'étudiants, et sans doute particulièrement la nouvelle génération émergente : elles intègrent toutes les facilités nécessaires aux usages numériques, y compris pour permettre le rechargement des équipements nomades. Elles mettent à disposition toute une gamme de mobiliers et de services de confort qui favoriseront un continuum entre le domicile et l'université. Elles encouragent les rencontres et les approches collaboratives, que ce soit en essayant de rapprocher documentation et pédagogie universitaire ou par la mise à disposition d'espaces adaptés à différents usages, et le développement de guichets d'assistance plus dynamiques et performants, offerts sur une multiplicité de canaux. Elles doivent enfin être conçues comme des espaces polyvalents qui se prolongent par l'ensemble des services numériques offerts et se caractérisent toujours par une amplitude horaire optimale.

"Cette richesse de l'offre s'exerce avec d'autant plus de succès auprès des étudiants qu'une grande amplitude horaire est la règle, y compris un service de nuit. $»^{2}$

2. Suzanne Jouguelet, Les Learning centres : un modèle international de bibliothèque intégrée à l'enseignement et à la recherche, Rapport n²009-022 de l'Inspection générale des bibliothèques, 
Si les bibliothèques se posent la question de l'adéquation de leurs horaires d'ouverture, cela est dû, bien sûr, d'abord aux effets des comparaisons internationales et aux volontés exprimées par les tutelles locales ou nationales. Mais ce sont également et surtout les nouvelles caractéristiques de nos étudiants qui imposent une disponibilité plus grande des espaces physiques pour répondre à de nouvelles formes de temporalités dans le travail étudiant, à de nouveaux besoins d'équipements liés à la connectivité permanente et à une conception de société moins hiérarchisée et plus collective. Ces politiques volontaristes ne peuvent donc faire l'économie d'une réflexion globale sur leurs services : ouvrir plus, mais comment, avec quels services et pour quels usagers ? C'est au final autant une question de contenus que d'amplitude. Dans ce contexte, les freins internes pour les projets d'extension d'horaires pourraient aussi être en partie analysés comme des difficultés d'appréhension intergénérationnelles, et les caractéristiques des étudiants actuels et futurs doivent être au centre des présentations du projet aux équipes, de façon à essayer (ce n'est pas simple) de partager au moins l'objectif avant d'en négocier les modalités. 


\section{3 \\ UNE BIBLIOTHĖQUE OUVERTE SUR DES INDIVIDUS EN CONSTRUCTION \\ par \\ Claude \\ Poissenot}

\section{JANVIER/FÉVRIER 2014 : LES BIBLIOTHĖQUES ONT FAIT L'ACTUALITÉ !}

La pétition lancée par Bibliothèques Sans Frontière a reçu un large accueil dans la population et les médias. Pour une fois les bibliothèques sont sorties des articles de la presse quotidienne régionale pour recevoir un écho dans les médias nationaux. Les professionnels qui regrettent régulièrement la faible visibilité de leur travail et de leurs établissements ont été entendus. Mais il convient de bien écouter ce que dit cette pétition. Cet événement s'est construit sur la thématique de l'ouverture et particulièrement sur l'extension des horaires d'ouverture et non sur la revendication pour davantage de documents. Le constat, fait depuis bien longtemps (y compris par les professionnels), de l'insuffisance des horaires d'ouverture se retrouve mis sur le devant de la scène par une mobilisation des citoyens fréquentant ou non des bibliothèques. C'est cette implication, cet enrôlement des usagers (réels ou potentiels) qui a valu à cette caractéristique française de devenir une lacune voire une anomalie. La situation d'équilibre entre une partie des élus (qui cherchent à limiter les dépenses de personnel) et une partie des professionnels (qui ne sont pas enthousiastes à l'ouverture le dimanche ou le soir) a été rompue et publiquement dénoncée. Un nouvel équilibre doit voir le jour dans lequel l'alliance d'une partie des professionnels (ceux qui s'accordent sur la définition de la bibliothèque par les publics) et de la plupart des publics déboucherait sur un élargissement des horaires.

Mais sur quoi repose cette aspiration conjointe de bibliothécaires et d'usagers à une plus grande ouverture des bibliothèques ? Au-delà des horaires, en quoi le thème de l'ouverture peut-il se décliner dans la bibliothèque ? 


\section{QUAND L'OUVERTURE DEVIENT LA NORME}

L'ouverture est à la mode et devient un élément de la modernité. On en perçoit le signe dans l'usage que nous faisons de sa traduction en anglais sous l'adjectif open. Le terme est repris, y compris à des fins commerciales, et signale une forme de disponibilité caractéristique de l'individu contemporain. Dans son usage au sein du monde informatique, il inclut aussi l'idée d'un partage au-delà des frontières qu'elles soient juridiques (data) ou techniques (source, office). Pourquoi donc faut-il être ouvert, open ?

Parce que nous sommes adaptables, la manière normative par laquelle nous sommes engagés à nous définir nous apparaît comme une évidence, une deuxième nature. Il nous apparaît spontané de faire preuve d'un esprit d'ouverture. Cela passe par des sanctions négatives (les enseignants peuvent disqualifier notre manque de curiosité et les parents plaisanter sur notre caractère timoré ou replié sur nous-même) mais très largement cette norme se transmet parce qu'elle donne lieu à des sanctions positives. Il est valorisé et valorisant de faire montre de largesse d'esprit, de curiosité et de souplesse. Le passager du TGV qui constate la présence d'une personne à la place qui lui est affectée peut apprécier les regards approbateurs des autres s'il fait part de sa disponibilité pour aller s'installer à une autre place. Mais d'où vient donc cette norme à laquelle nous sommes soumis en tant qu'individu et qui s'impose aux institutions par un effet de contamination? L'injonction à l'ouverture est le produit du processus d'individualisation dans lequel nos sociétés occidentales sont engagées ${ }^{1}$. Nous sommes invités à nous définir non à partir d'appartenances extérieures et imposées mais à partir de ce qui forme notre singularité : notre histoire et notre sensibilité. Nous nous revendiquons ainsi comme des personnes par-delà les statuts que nous occupons. Notre existence ne se réduit ainsi pas à une existence prédéterminée, à un destin tout tracé qui correspondrait à une succession d'étapes prédéfinies. Au contraire, nous pensons notre vie comme un ensemble de potentialités parmi lesquelles nos choix nous

1. Christian Le Bart, L'individualisation, Paris, Presses de Sciences Po, 2009 (coll. Références. Sociétés en mouvement). 
conduisent à n'en vivre qu'un nombre restreint ${ }^{2}$. Bien sûr, l'univers des possibles n'est pas également distribué. Les inégalités de ressources économiques, sociales ou culturelles réduisent ou augmentent le spectre des expériences potentielles. Le fait de disposer de revenus élevés élargit les opportunités de voyages, de rencontres et, donc, d'une expérience variée de l'existence. De même, la familiarité avec des formes diverses d'expression artistique et la capacité à s'y repérer donnent l'habitude d'une palette étendue de modes de vie. Mais si le fait de disposer de ressources facilite l'actualisation de la norme d'ouverture, celle-ci s'impose à tous et chacun peut trouver (y compris dans le cadre d'une vie contrainte par la faiblesse du niveau de vie) des marges infimes qui lui fournissent le sentiment, partiellement justifié, de maîtriser une part de son existence. Nous souhaitons acquérir le sentiment d'être l'auteur de notre propre vie. Cela peut passer par des pratiques de consommation mais aussi par des manières de se raconter, par des choix éthiques ${ }^{3}$ ou encore par des sensations ${ }^{4}$.

\section{LES BIBLIOTHĖQUES ET L'OUVERTURE}

L'injonction à l'ouverture passe des membres de notre société aux institutions qui la composent. Dans les discours, notre société se veut « ouverte » c'est-à-dire être un cadre à l'intérieur duquel les individus trouvent des occasions d'exprimer leur singularité par-delà les frontières nationales, statutaires, sociales, etc. Et c'est clairement dans cette filiation que notre société valorise les notions de "souplesse » ou de " mobilité ». Dès lors, les institutions ne sont plus seulement dans la fonction d'institutionnaliser mais doivent aussi "s'adapter », suivre des évolutions qui les entourent.

2. Dans son roman Lisières (Flammarion, 2012), Olivier Adam met en scène un ami du narrateur qui, comme lui, est issu d'une famille de milieu populaire et qui, suite à des études supérieures interrompues faute d'investissement personnel, fait l'expérience durable de la précarité. Le destin peu favorable de ce personnage est interprété par lui pas tant en terme de structure sociale inégalitaire que de « chance » que ses parents lui ont fourni et qu'il n'a pas su saisir.

3. Dans le roman d'Edouard Louis, En finir avec Eddy Bellegueule, (Seuil, 2014), le narrateur explique comment son père s'impose avec force le refus de frapper ses enfants, ayant lui-même été victime de cette violence pourtant assez banale dans le monde ouvrier dans lequel se déroule ce récit.

4. Voir les pages que Jean-Claude Kaufmann consacre à la dimension sensible de la construction de l'identité dans L'invention de soi : une théorie de l'identité, Paris, Armand Colin, 2004 (coll. Individu et société). 
Et c'est ainsi par exemple que le mariage peut prendre la forme du PACS ou s'ouvrir aux personnes de même sexe. Le glissement de la notion d'« administration publique » à celle de " service public » consacre l'idée d'une adaptation à l'évolution de la définition des citoyens. Ceux-ci ne sont plus seulement des " administrés » passifs, ils ont droit à un service qui tient compte de leur singularité et de leurs aspirations. Le site gouvernemental Vie-publique.fr définit le régime juridique des services publics ${ }^{5}$ à travers le principe de " mutabilité (adaptabilité) » en plus de ceux de « continuité » et d'« égalité ». L'enfermement des administrations dans leurs routines anciennes ne serait plus à l'ordre du jour et, au contraire, heurterait ce principe fortement affirmé aujourd'hui.

Comme toutes les administrations devenues services publics, les bibliothèques sont concernées par cette mutation. Mais en quoi contribuentelles ou peuvent-elles contribuer à l'ouverture des individus?

\section{DES INDIVIDUS ENTRE OUVERTURE ET FERMETURE}

Avant de montrer par quelles dimensions de leur offre, les bibliothèques peuvent contribuer à l'ouverture des individus, il faut présenter cette idée en l'inscrivant dans une réflexion plus large sur le travail identitaire. Puisque les individus ne sont pas définis (entièrement) de l'extérieur, ils doivent se construire dans un processus qui alterne entre ouverture et fermeture.

Les moments d'ouverture sont plus rares mais plus intenses. Ils correspondent à une phase au cours de laquelle les individus développent des modalités de définition qu'ils n'avaient pas explorées ou pas actualisées depuis longtemps. Ces moments peuvent être le produit d'une sensation fugitive $^{6}$ et agréable (découvrir le plaisir du repassage...). Ils peuvent aussi être le résultat d'une réflexion plus lente et raisonnée, une délibération conduisant à une reformulation de la vision de soi (je renonce à ma formation de personnel de restauration). Ces phases d'ouverture se traduisent par des remaniements dans les relations de sociabilité comme dans les pratiques culturelles ou les visions du monde. Un « nouveau moi »

5. Voir le site : < http://www.vie-publique.fr/th/glossaire/service-public.html >.

6. Voir aussi Jean-Claude Kaufmann, Quand je est un autre : pourquoi et comment ça change en nous, Paris, Armand Colin, 2008 (coll. Individu et société). 
voit le jour et prend la place d'un « ancien moi » dont les traces vont peu à peu disparaître.

En effet, il faut ensuite que la nouvelle version du « moi » se stabilise. On entre alors dans une phase de fermeture dans laquelle se construit une nouvelle " normalité ", un ensemble d'évidences qui vont refermer le couvercle de la boîte à questions. De nouveaux lieux, de nouveaux visages vont devenir familiers et nous conforter dans notre identité. Des sensations vont s'inscrire dans des habitudes, s'affiner et devenir une source prévisible de plaisirs. Des gestes seront intériorisés dans des routines rassurantes et confirmatoires. Un récit de soi se stabilise avec des moments perçus comme clés et cette « histoire que chacun se raconte » est partagée par ceux qui nous entourent pour conduire à l'évidence de notre identité. La bibliothèque occupe ou peut occuper une place dans ce double mouvement d'ouverture et fermeture, constitutif du travail identitaire. Bien sûr, la phase d'ouverture apparaît le plus visiblement mais elle ne doit pas masquer le temps de fermeture qui lui est indissociable : pas d'identité sans stabilisation du moi. Il faut donc prêter intérêt à ces deux dimensions.

\section{L'OUVERTURE À QUELS HORAIRES : LE MATIN, LE SOIR, LE DIMANCHE, LE LUNDI, LES VACANCES?}

La question des horaires d'ouverture des bibliothèques a bien sûr à voir avec le travail identitaire dans la mesure où c'est aux moments où les usagers sont disponibles qu'ils peuvent s'y rendre dans cet objectif. Quelles que soient les pratiques qu'ils feront de la bibliothèque, il faut d'abord que les horaires d'ouverture coïncident avec leur rythme de vie.

Le cadre professionnel impose largement ses contraintes et, depuis longtemps par exemple, on sait que la fermeture très fréquente des bibliothèques le lundi forme un frein important à la fréquentation des commerçants qui ne peuvent s'y rendre le samedi quand ils travaillent. De même, les ouvriers qui travaillent l'après-midi ou le soir ne peuvent être que pénalisés si les bibliothèques sont fermées le matin comme c'est largement le cas dans le réseau de la Ville de Paris par exemple. Mais les contraintes ne se limitent pas au monde professionnel. L'ouverture des bibliothèques pendant les vacances est souvent couronnée de succès parce 
qu'elle permet la venue d'élèves qui ne peuvent faire leurs devoirs à leur domicile ou venir les semaines d'école.

Le rythme de vie est aussi défini par les activités familiales et amicales. C'est là notamment que se trouve particulièrement la justification de l'ouverture du dimanche. Alors qu'il est difficile d'harmoniser les temps de tous les membres de la famille, le dimanche apparaît comme un moment particulièrement propice pour rassembler tous les membres de la famille. Il serait heureux que la bibliothèque puisse être le cadre de cette appropriation familiale. C'est en devenant le lieu d'une activité commune que la bibliothèque peut devenir familière à tous et particulièrement aux enfants. Rappelons que la pérennité de la fréquentation juvénile est nettement améliorée quand les parents sont aussi fréquentants*. Outre la question du service rendu, se trouve donc un enjeu stratégique pour la bibliothèque et sa capacité à maintenir son attractivité auprès des nouvelles générations. Mais le dimanche peut être aussi le moment d'une appropriation personnelle ou amicale par opposition à une sociabilité familiale. Dans une enquête réalisée en 2006 sur les publics de la médiathèque de Vandoeuvre-lès-Nancy, on proposait une question sur les créneaux d'ouverture souhaités. Si le dimanche arrivait légèrement devant l'ouverture en matinée ou le lundi (respectivement $33 \%$, $31 \%$ et $25 \%$ ), c'était notablement le cas chez les usagers (plutôt jeunes) qui étaient venus avec au moins un(e) ami(e) (48\% contre $30 \%$ pour ceux qui étaient venus seuls). L'enquête révèle en outre que cet usage amical et dominical serait particulièrement tourné vers le travail sur place avec ses propres documents (34 \%) ou avec les documents de la médiathèque (20\%) mais aussi orienté vers l'objectif de passer du temps (20\%). Par son ouverture le dimanche, la médiathèque serait ainsi le support d'une appropriation collective en différenciation par rapport à un usage familial.

\section{L'OUVERTURE : QUELS ESPACES ?}

Ouvrir davantage certes, mais pour quel(s) espaces ? En quoi le lieu bibliothèque participe-t-il à l'ouverture des individus ? Plus particulièrement, en quoi cet espace public peut-il contribuer à la construction de l'identité personnelle? 
La bibliothèque peut être un espace propice dans la phase d'ouverture du travail identitaire. Les individus peuvent trouver un cadre à une nouvelle définition d'eux-mêmes. Par exemple, pour certains jeunes de milieu populaire, la bibliothèque remplit cette fonction d'incarner un lieu conforme à la manière dont ils souhaitent se définir, à savoir par leur implication dans leurs études. Outre le cadre de travail adapté à cette activité, la bibliothèque offre aussi un symbole de la valeur accordée au monde du livre, de la lecture et, en cela, à l'univers scolaire. Une partie des usagers des bibliothèques est donc dans une logique que nous avons qualifiée "d'invention de soi " ${ }^{7}$ qui se manifeste par une appropriation de l'espace comme cadre d'une identité personnelle autre de celle qui leur est reconnue par ailleurs. Cette manière de se définir ne sera pas nécessairement confirmée par d'autres interactions même si elle peut être le prélude à une nouvelle identité validée par d'autres. L'espace de la bibliothèque forme donc le cadre à cette invention de soi. On se souvient par exemple ${ }^{8}$ de cet Africain qui avait investi la Bibliothèque publique d'information (Bpi) et qui rédigeait sur place des " ouvrages » et s'était constitué une vie sociale dans ce lieu qui était devenu une sorte d'espace personnel.

Mais la bibliothèque offre aussi un espace propice à la fermeture de soi. Les identités ont besoin d'être confirmées pour être stabilisées. Certains usagers trouvent à la bibliothèque le lieu pour recevoir une confirmation y compris silencieuse. L'enquête sur les pauvres à la $\mathrm{Bpi}^{9}$ a permis de relever que, pour une partie d'entre eux, le séjour à la bibliothèque est un moyen pour maintenir une version de soi positive. Le regard des autres n'est pas stigmatisant, ils ne sont pas " pauvres », ils sont " usagers » comme les autres. Ces " moins » pauvres ont un regard sévère sur ceux qui ont basculé du côté d'une pauvreté visible. Cette logique de " confirmation de soi » sous-tend une partie de la fréquentation y compris durable et répétée d'une partie des usagers.

7. Claude Poissenot, Sophie Ranjard, Usages des bibliothèques, Villeurbanne, Presses de l'enssib, 2005.

8. Jean-Michel Cretin (réal.), Les habitués, Paris, Productions 108 - Bibliothèque publique d’information/Centre national d'art contemporain Georges Pompidou - Centre audiovisuel de Paris, 1998.

9. Serge Paugam, Camila Giorgetti, Des pauvres à la bibliothèque : enquête au Centre Pompidou, Paris, Presses universitaires de France, 2013 (coll. Le lien social). 
La manière même dont la bibliothèque met en forme son espace peut se révéler un support précieux pour cette confirmation de soi. Le recours des bibliothèques (notamment anglo-saxonnes) à des espaces dédiés à des " communautés » nous paraît particulièrement exemplaire. Les adolescents en quête d'affirmation de leur personnalité adolescente se retrouveront volontiers dans une "teen zone » ou les personnes homosexuelles en formation trouveront un soutien dans le rayon " gays et lesbiennes ». La bibliothèque ne les enferme pas dans ces espaces et donc dans leur communauté mais leur propose un support utile à la construction de leur identité.

\section{L'OUVERTURE AUX PUBLICS}

En tant qu'espace public, la bibliothèque accueille la population dans sa diversité. En cela, elle offre à ses visiteurs de retrouver ceux qui leur ressemblent ou, au contraire, ceux qui leur sont différents, qu'ils soient conformes à ce que l'on voudrait être, ou inversement, qu'ils soient le portrait de ceux par rapport auxquels ils se construisent. À tous, elle permet de confronter des existences et des modes de vie, des goûts culturels et des manières de se tenir.

Certains visiteurs vont apprécier de retrouver leurs homologues ou " leur » bibliothécaire favori. Ils s'inscriront dans une logique de confirmation de soi. À l'opposé, d'autres apprécieront d'être surpris par des rencontres improbables, des événements imprévus (on pense avec émotion à l'initiative du bibliothécaire musicale de la médiathèque de Limoges qui avait célébré la disparition de Michael Jackson par une chorégraphie impromptue peu avant la fermeture de l'établissement à la surprise et la satisfaction de nombreux visiteurs $)^{10}$. Ils puiseront dans ces occasions des sources d'ouverture.

Bien sûr, cette fonction d'ouverture par les publics dépend largement de la structure de la fréquentation des bibliothèques. La surreprésentation trop marquée d'une population par rapport aux autres favorisera plutôt la fermeture. Il en va ainsi d'une fréquentation trop massive des jeunes, des 
personnes âgées, des enseignants, etc. Au contraire, les établissements parvenant à rassembler une diversité de profils de visiteurs, remplissent une fonction d'ouverture sur autrui. De surcroît, ils parviennent à " faire société ", c'est-à-dire, à mettre ensemble des citoyens libres (de fréquenter cet espace public) et différents.

\section{UNE CULTURE OUVERTE}

L'ouverture sur la diversité des publics ne peut intervenir sans une ouverture sur la diversité de leurs références et plus largement de leurs manières de vivre. Dans ce cas, l'ouverture concerne à la fois la diversité des formes d'expression mais aussi leur niveau de " qualité ». Les bibliothèques françaises ont inscrit leurs pas dans le sillon de l'action des équipements culturels. Elles ont eu (et ont encore parfois) à cœur de sélectionner les documents (mais aussi les animations) en fonction d'une vision encyclopédique de la connaissance mais aussi à partir d'une hiérarchisation des œuvres. Certaines relèvent davantage d'une vision dans laquelle les critères de qualité (eux-mêmes sujets à changement) sont présents. Elles sont le fruit d'une création personnelle, d'un " auteur », renvoient à l'histoire de l'art, à une recherche formelle alors que d'autres sont présentées comme une production quasi industrielle, anonymes et dédiées au seul délassement de lecteurs éloignés d'une recherche de « la » littérature ou de « la » connaissance.

Cette conception de la bibliothèque a constitué une formidable ouverture pour les publics qui ne partageaient pas ces références et pouvaient ainsi accéder à des documents qui quittaient le monopole des catégories dominantes pour concerner des catégories populaires. Mais si le projet de cette démocratisation de la culture a été généreux, il est désormais possible d'en faire la critique. Il a aussi réellement constitué une fermeture sur cette culture légitime. Les catégories de population à distance de ces références et de cette vision de la culture ont largement délaissé les bibliothèques. N'étant pas à leur image et ne souhaitant pas renoncer à leur univers culturel (fût-il populaire), elles ont renoncé à s'approprier les bibliothèques. Les chiffres de fréquentation dressent ce constat avec persistance et les ouvriers, les agriculteurs sont largement sous-représentés. La propension à venir en bibliothèque est directement corrélée 
au niveau d'études, ce qui apparaît comme le signe majeur des limites de l'ouverture de cette conception de la bibliothèque qui s'adresse à ceux qui la partagent ou à ceux qui acceptent de s'y soumettre.

Une bibliothèque réellement ouverte suppose donc une rénovation de la conception de la culture privilégiant la diversité sur la hiérarchisation. La population est largement prête à cette mutation et la plupart des bibliothécaires aussi, ce qui ouvre la voie à cette évolution.

\section{DU SERVICE PUBLIC AU SERVICE AUX PUBLICS : L'USAGER CONCRET*}

Si les citoyens réclament une plus grande ouverture des bibliothèques, c'est que l'ouverture est une valeur cardinale de notre société. Chacun revendique le droit d'être lui-même, celui d'être l'auteur de sa propre vie. L'ouverture n'est plus tant le fait d'être à l'écoute du monde qu'il s'agirait de connaître que d'être à l'écoute de soi-même. Face à ce changement, la bibliothèque ne peut plus se définir centralement à travers la mise à disposition de documents visant à " ouvrir » les usagers. Les citoyens entendent reprendre la main sur leur propre destin et les documents (moins indispensables à l'heure de l'information numérisée) ne constituent un support que pour autant qu'ils le décident. Dès lors, les bibliothèques passent du service public au service aux publics ${ }^{11}$. Ceux-ci demandent à ce qu'elles soient ouvertes quand ils sont disponibles. Ils aspirent à trouver des documents mais aussi plus largement un cadre qui vont leur permettre de construire leur identité personnelle en "inventant » une version d'eux-mêmes ou en " confirmant » une version déjà existante. C'est en s'inscrivant dans cette aspiration de la population que les bibliothèques sont à même de reconstruire un rapport d'adhésion avec elle. Cette reformulation sera à même d'assurer aux bibliothèques une pérennité à l'heure où la dématérialisation et la restriction des finances publiques (y compris locales) menacent leur existence.

11. Claude Poissenot, "L'irruption de l'usager concret ", BIBLIOthèque(s) - Revue de l'Association des bibliothécaires de France, 2010, $\mathrm{n}^{\circ}$ 53/54, pp. 24-27. 


\section{LES MOTS DE L'OUVERTURE : NUAGE DE MOTS} À PARTIR DES COMMENTAIRES DE LA PÉTITION

\section{FIGURE}

« OUVRONS PLUS LES BIBLIOTHĖQUES »

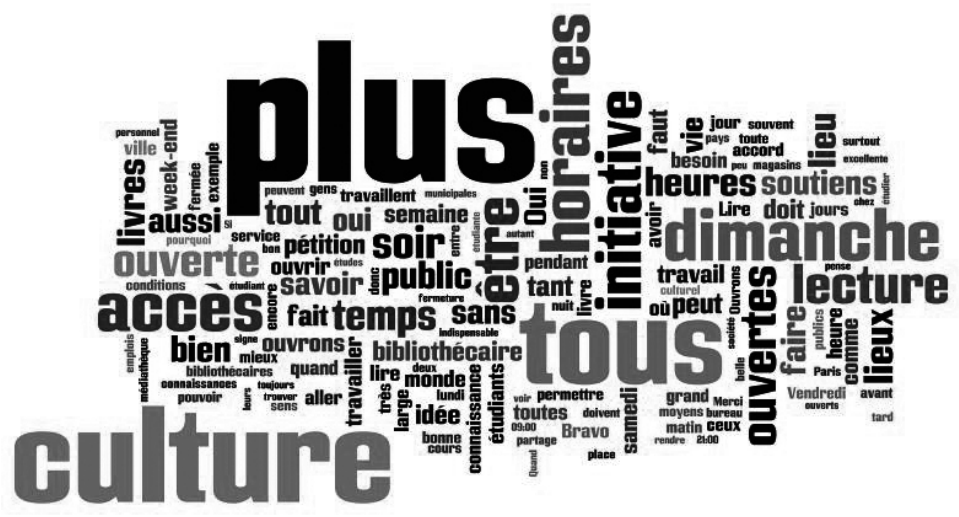

Corpus constitué entre le 10 janvier et le 8 février 2014. Environ 575 messages courts ont été recueillis sur le site Ouvrons $+{ }^{12} \ldots$ Ils apparaissaient peu de temps sur la page d'accueil du site et ont donc été copiés-collés. Certains messages trop longs n'apparaissaient pas en entier. Nous avons pris en compte uniquement les messages qui ne se résumaient pas à un "bravo » ou « merci ». 
OUVRIR OPPORTUNÉMENT :

LA MÉDIATHĖQUE DU

KREMLIN-BICÊTRE

\section{4}

\section{UNE RELATION AU PUBLIC REDÉFINIE}

" Ouvrir plus » sans augmentation proportionnelle de personnel, comme c'est bien souvent le cas, implique de dégager du temps aux professionnels pour qu'ils puissent accueillir le public. En d'autres termes, il s'agit de remettre en cause les activités gourmandes en personnel qui n'ont pas d'impact sur les services pour le public. Au Kremlin-Bicêtre, pour ce faire, nous avons d'abord mis en application les enseignements de nos pairs dans ce domaine : l'architecte a été incitée à construire de grands plateaux décloisonnés allégeant les impondérables de surveillance, le traitement du document a été rationalisé (récupération de notices, notamment) et l'accueil des groupes et les actions spécifiques sont autant que possible programmées pendant les heures d'ouverture au public, afin de garantir l'efficacité des plages de travail interne de préparation. Puis nous essayons d'innover ${ }^{1}$, non pas tant pour libérer davantage de temps d'ouverture, encore que..., mais surtout pour prendre le temps d'investir qualitativement le service au contact du public.

Dès lors que le public est autonome pour emprunter et rendre les documents, dès lors que le bibliothécaire est davantage au contact de l'usager, une question se pose : que faire, avec et pour lui ?

Cette question rejoint plus largement celle posée nationalement par la volonté de modernisation du service public. Outre la mise en place d'une culture de l'évaluation orientée sur des objectifs discutables à bien des égards, la part heureuse de l'initiative consiste à simplifier l'accès

1. Pour réduire le temps consacré à la constitution des collections où la réelle plus-value du médiathécaire se porte sur l'élaboration d'une politique documentaire et non pas sur un choix titre à titre, la médiathèque a développé des partenariats avec des libraires et dévédécaires afin de bénéficier au maximum de leur expertise. De ce fait, ils nous proposent une partie des titres à partir des grandes orientations de nos collections. 
des citoyens à leur administration, à assurer une réactivité qui fait trop souvent défaut et à donner plus de transparence aux procédures, soit à redonner du sens au service public pour qu'il apparaisse véritablement comme le "service du public ».

Concrètement, ce souci s'est traduit au Kremlin-Bicêtre par l'engagement dans une démarche de certification ISO 9001 pour l'amélioration de l'accueil du public. Le député-maire, Jean-Luc Laurent, l'exprime en ces termes : " Ma vision pour la ville, dit-il, est de faire de la mairie une " maison commune » où la demande est comprise, bien traitée et considérée dans sa globalité $»^{2}$. La qualité d'accueil s'apparente alors à un combat républicain, le même qui a motivé la création de la médiathèque ${ }^{3}$ : « Dans un climat de crise économique associé aux projets de réformes des collectivités territoriales et à leur financement, nous devons être encore davantage à l'écoute des habitants en recherchant sans cesse les pistes d'amélioration de nos services publics. »

Dans ce contexte, le désir de libérer du temps pour accueillir le public est directement associé aux principes énoncés par le maire : créer de la richesse sociale en simplifiant l'utilisation de l'équipement, en étant réactif aux besoins de la population et en reconnaissant les droits de chacun à bénéficier des services de la médiathèque.

À l'aune de ces discours, les professionnels du Kremlin-Bicêtre ont reconsidéré le fonctionnement de l'équipement. Il est alors apparu que l'organisation de la médiathèque ne permettait pas, bien souvent, d'actualiser correctement tous ces principes.

Au cours de réflexions menées de manière collective, les dysfonctionnements ont été recensés. Ils portaient à la fois sur la disponibilité des documents, sur le comportement du public, sur les compétences des bibliothécaires. Ce travail a abouti, pour une part, à la formulation d'« actions correctives » mises en place progressivement et durablement grâce à un

2. Le Manuel qualité de la ville du Kremlin-Bicêtre décrit en ces termes la politique qualité (voir p. 7) : «Simplifier les démarches administratives pour les usagers ; maintenir et développer une réactivité de nos services accueil ; créer et entretenir des valeurs communes et des modèles de comportement fondés sur l'équité. "

3. La médiathèque L'Écho a été construite en face d'un centre d'affaires prolongé d'un centre commercial afin d'équilibrer l'offre de services à la population par un établissement public, ouvert à tous et gratuit. < http://lecho.kremlinbicetre.fr/exploitation/ > . 
tableau de bord faisant état des nouveaux problèmes. Il a été assorti de l'élaboration d' " un socle de compétences communes » permettant à tous de dispenser une qualité homogène (constante) de services à l'ensemble du public.

\section{AU-DELÀ DE LA TECHNIQUE : ACCOMPAGNER L'USAGER}

Très prosaïquement, le socle de compétences communes vise à assurer la maîtrise de tous les outils constituant l'offre de base de la médiathèque : chaque membre de l'équipe saura se servir du photocopieur, du logiciel d'utilisation des postes multimédias, des imprimantes de tickets, des services du portail de la médiathèque. Dans un autre registre, chaque membre de l'équipe saura s'adapter aux besoins des différents lecteurs. L'ambition consiste alors à développer des repères communs, issus de l'expérience de chacun, pour comprendre les besoins des différents publics et y répondre avec pertinence : se baisser lorsqu'on parle à un enfant ; se déplacer pour demander le silence ; aider l'assistante maternelle à canaliser l'énergie des enfants dont elle a la garde ; fixer des limites pour être à même de conclure avec un lecteur trop accaparant afin de se rendre disponible pour les autres ; pouvoir faire découvrir des ressources électroniques plus variées que « bip bip » aux minots hypnotisés par les écrans. En bref, mesurer quand on fait porter au public les conséquences d'un insuffisant souci de lui...

Les philosophes du care expliquent que les métiers de l'accueil, de l'accompagnement ou de la médiation, exercés notamment par le bibliothécaire aujourd'hui, consistent à se mettre au service de la vulnérabilité du public. Vulnérable parce que beaucoup d'usagers ont besoin du concours d'un tiers pour faire aboutir leur demande (explicite ou implicite). Et il y a deux façons de répondre à cette vulnérabilité.

En faisant en sorte que la médiathèque ne mette pas l'usager en situation de vulnérabilité d'abord, c'est-à-dire en limitant les difficultés pour le public (horaires d'ouverture impossibles à mémoriser, classement abscons des ouvrages, etc.). C'est en identifiant les dysfonctionnements qu'un engagement dans une démarche qualité du type ISO 9001 pourra donner des fruits. Puis, dans la relation directe à l'usager, en étant attentif à sa vulnérabilité - celle qui n'est pas induite par le fonctionnement de l'établissement mais qui lui est propre -, en la prenant en compte. Vouloir développer 
des compétences partagées prend alors tout son sens (trouver le ton juste pour transmettre les règles, aider un lecteur à formuler clairement sa demande, proposer l'aménagement d'un service qui prendrait en compte une vulnérabilité non considérée...).

\section{LISIBILITÉ DES HORAIRES ET OUVERTURES EXCEPTIONNELLES : DES OPPORTUNITÉS À SAISIR}

L'élargissement des horaires d'ouverture conduit le public à fréquenter plus spontanément la médiathèque - et l'ouverture le dimanche est un pas décisif en ce sens. Néanmoins, sans être ouverte le dimanche, la médiathèque est fréquentée par un public nombreux et assidu.

Au Kremlin-Bicêtre, il a été privilégié la lisibilité des horaires d'ouverture :

- ouverture à midi en semaine (mardi, jeudi et vendredi) et à $10 \mathrm{~h}$ le mercredi et le samedi ;

- fermeture à $19 \mathrm{~h}$ du mardi au samedi.

Midi pour être accessible aux nombreux actifs présents à proximité de la médiathèque, et $19 \mathrm{~h}$ pour permettre aux Kremlinois ne travaillant pas sur le territoire de faire un saut en sortant du métro, ou du lycée - plutôt que l’impact d'une nocturne en semaine (à nos yeux moins pertinent dans l'environnement géographique et social), ou une ouverture le dimanche qui aurait supposé des moyens financiers trop incertains au moment de la création. En 18 mois d'existence, la médiathèque est devenue un lieu incontournable de l'offre culturelle du territoire. Cette adhésion du public a conduit l'établissement à adapter et élargir ponctuellement ses horaires d'ouverture pour des événements locaux ou nationaux particulièrement en résonance avec ses missions.

Le premier de ces événements a lieu fin janvier lors du festival des cultures russes contemporaines, "RussenKo ». À cette occasion, la médiathèque se transforme pour accueillir jusqu'à 2000 visiteurs qui viennent participer aux rencontres, expositions et spectacles des journées littéraires. Elle ouvre alors le samedi jusque tard dans la soirée et le dimanche toute la journée. 
Un peu plus tard dans l'année, la médiathèque L'Écho renouvelle l'expérience en augmentant de $20 \mathrm{~h}$ son amplitude horaire la semaine précédant les épreuves du baccalauréat afin de proposer aux lycéens un cadre propice à l'étude. Les espaces publics sont alors réaménagés pour offrir 160 places de travail. Ce dispositif en faveur de la réussite scolaire est au départ une initiative de la bibliothèque de la ville de Fresnes; fort de son intérêt pour le public et du succès qu'il a d'emblée rencontré, de nombreuses médiathèques du Val-de-Marne ${ }^{4}$ l'ont mis en place depuis. Aujourd'hui, cette opération est suivie par la presse ${ }^{5}$ et s'enrichit chaque année : programmation de séances de relaxation, de cours particuliers dispensés par des bénévoles, ou encore de moments conviviaux...

Avec ces exemples, l'élargissement des horaires d'ouverture permet la création de nouveaux services.

En étant davantage mobilisés du fait de cette amplitude horaire nouvelle, et davantage disponibles grâce à l'automatisation, les membres de l'équipe ont été amenés à repenser leurs tâches en faveur d'une relation plus directe avec le public. Juste retour de cette évolution, l'augmentation significative de la fréquentation de la médiathèque a donné une envergure tout autre à cet équipement culturel qui peut désormais s'autoriser des écarts pour ouvrir en grand ses portes à l'occasion d'événements ou de manifestations sortant de l'ordinaire.

Ainsi, on voit comment ce changement a impliqué une réorganisation du travail et une attitude nouvelle des professionnels en faveur de l'amélioration de la qualité d'accueil. Sans conteste, ces expériences dessinent un cercle vertueux où « ouvrir plus » conduit à « ouvrir mieux ».

4. L'association des bibliothèques du Val-de-Marne réunit les bilans des établissements organisant cette opération où l'on peut se rendre compte de l'impact très impressionnant de ce service exceptionnel sur le public ciblé : < http://www.valmedia94.fr/boite-a-idee/dispositifs-revisionsen-2014-dans-nos-mediatheques >.

5. Articles dans Le Parisien et reportages de BFM TV par exemple. 


\section{5}

\section{DE LA BIBLIOTHĖQUE AU LEARNING CENTER (LILLE 3) : UN ACCUEIL RENOUVELÉ POUR TOUS LES PUBLICS}

Le service commun de la documentation (SCD) de Lille 3 dessert une université arts, langues, lettres, sciences humaines et sociales de 18000 étudiants et 800 enseignants, implantée à Villeneuve-d'Ascq. Il comprend la bibliothèque universitaire $(B U)$ et vingt-deux bibliothèques d'UFR ou de laboratoires de recherche. Un SIGB et un catalogue communs ainsi qu'un service de catalogage centralisé structurent ce réseau qui emploie une centaine d'agents et conserve 1,2 million de documents.

La BU, construite en 1974, est un bâtiment de $17000 \mathrm{~m}^{2}$, dont $12000 \mathrm{~m}^{2}$ de services publics et internes et $5000 \mathrm{~m}^{2}$ de magasin de conservation, 800000 documents soit 23 kilomètres linéaires de collections, dont 200000 documents en libre accès, et 1200 places assises.

L'université Lille 3 et la Région Nord-Pas de Calais portent un ambitieux projet de Learning center (LC) inscrit dans le cadre d'un projet régional de maillage du territoire d'un réseau de quatre LC thématiques ${ }^{1}$ (" les faits religieux " à l'abbaye de Vaucelles près de Cambrai, "l'innovation » à Lille 1, "l'archéologie, l'égyptologie et les SHS » à Lille 3, enfin « la ville durable » à Dunkerque) ayant vocation à accueillir tous les publics, prenant en compte les nouveaux modes d'enseignement, la culture numérique et la formation tout au long de la vie. Le projet de Lille 3 bénéficie d'un financement Plan Campus et Contrat de plan État-Région (CPER).

Ce projet transversal et structurant, inscrit dans le projet d'établissement de l'université et concernant l'ensemble du campus, comprend une programmation de valorisation de la recherche débutée en janvier $2011^{2}$, une bibliothèque universitaire repensée et mise au service d'une pédagogie renouvelée. Dans ce cadre, le SCD a mené des études destinées à mieux

1. < https://www.nordpasdecalais.fr/jcms/c_5311/learning-centers >.

2. < http://learningcenters.nordpasdecalais.fr/archeologie-egyptologie/ >. 
connaître le fonctionnement du bâtiment actuel afin de nourrir la programmation. Les principaux indicateurs relevés, quantitatifs et qualitatifs, concernent les publics et les espaces y compris les services associés.

Le concept de Learning centre implique un ancrage physique fort dans un lieu stratégique en termes de visibilité, d'emplacement, de fréquentation et d'amplitude d'ouverture.

\section{MIEUX CONNAÎTRE LES PUBLICS ET LES USAGES}

\section{LE NOMBRE D'ENTRÉES}

Les statistiques de fréquentation des bibliothèques universitaires (enquête RAP/ministère de l'Enseignement supérieur et de la Recherche) comptabilisent le nombre d'entrées dans le bâtiment. À la BU de Lille 3, on dénombre environ 500000 entrées par an, 451853 entrées en 2013, soit 2061 entrées par jour en moyenne. Cet indicateur ne reflète que partiellement l'activité de la bibliothèque. Les études nécessaires à la programmation ont poussé à vouloir en savoir plus sur l'occupation des espaces. Faute d'un logiciel capable de rendre compte du nombre d'usagers présents dans le bâtiment, un système de relevé manuel (avec compteur mécanique) à heures ouvrées, confié aux agents en service public dans les différents espaces, a été mis en place entre septembre 2013 et juin 2014. Les indicateurs recueillis font état des tendances suivantes : on compte 600 usagers présents aux heures de pointe. Les périodes les plus chargées de la journée sont la fin de matinée et le milieu d'après-midi. 


\section{GRAPHIQUE PRÉSENCE DES USAGERS À LA BU SELON LES HEURES}

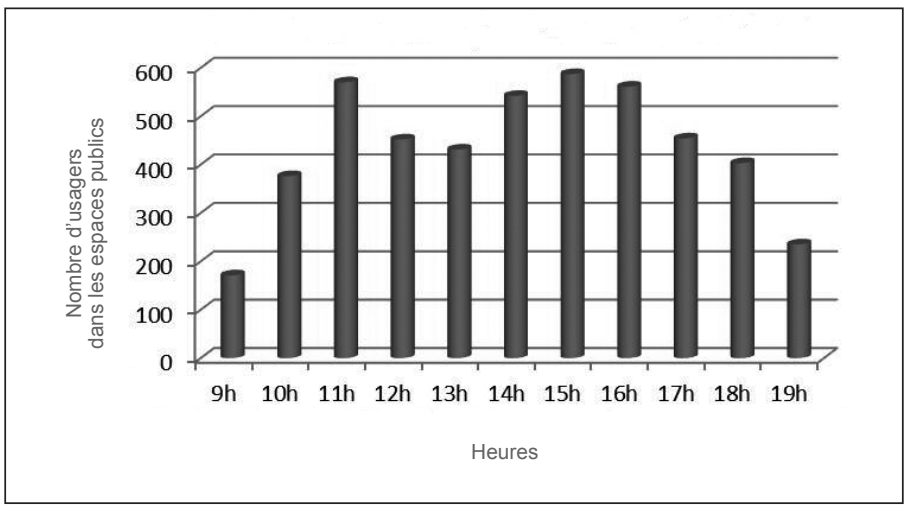

Sur la période de septembre à fin-mai, les pics de fréquentation s'enregistrent la première quinzaine de décembre, la semaine de réouverture en janvier et à la mi-mai. Ces périodes correspondent aux périodes de révisions et des évaluations des $1^{\text {er }}$ et $2^{\mathrm{e}}$ semestres.

Ce comptage a permis d'évaluer le nombre de places de travail souhaitées dans le futur bâtiment, en tenant compte de ces chiffres et de l'effet d'appel d'un nouvel équipement.

\section{GRAPHIQUE OCCUPATION DES ESPACES DE TRAVAIL DE LA BU}

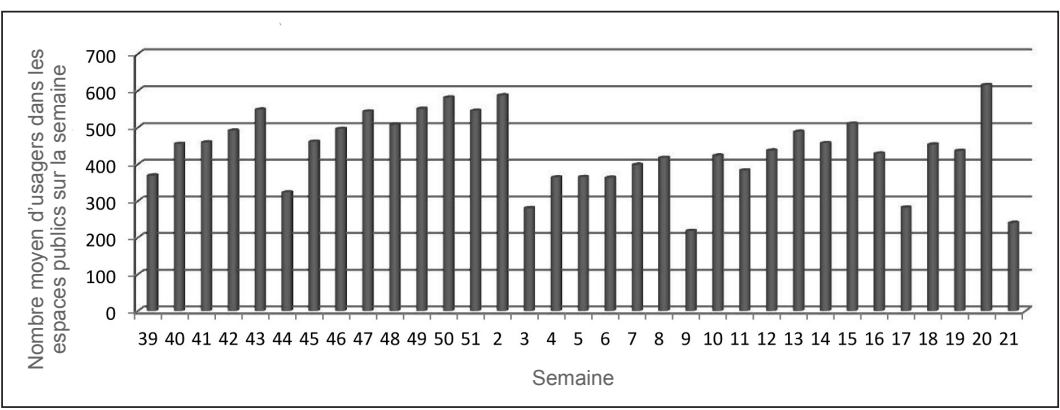




\section{Les lecteurs actifs}

Le recours à la documentation par les étudiants se mesure aussi par la notion de lecteurs actifs ${ }^{3}$ : lecteurs inscrits à la bibliothèque ayant effectué au moins un emprunt. En 2013, 1 étudiant de licence sur 3, 2 étudiants de master sur 3, 1 étudiant de doctorat sur 2 sont lecteurs actifs de la BU. L'effort doit notamment porter sur les étudiants de licence et plus particulièrement la $1^{\text {re }}$ année. Un des objectifs du LC est d'aider encore à la réussite étudiante. En 2012, une étude menée par le SICD et l'Observatoire de la vie étudiante (OVE) de l'université de Toulouse démontre une corrélation entre l'utilisation des bibliothèques, mesurée par l'emprunt de livres, et la réussite des étudiants au cours des trois années du cycle licence ${ }^{4}$.

\section{QUE FONT LES USAGERS À LA BU ?}

Début 2012, une enquête ${ }^{5}$ qualitative est menée, consistant à observer les usagers de la BU tant dans leurs postures que dans leurs activités et à relier, dans la mesure du possible, cette observation avec les services offerts. Cette enquête a permis de relever deux tendances majeures sur les usages du public et de confirmer le travail à mener pour faire de la BU un véritable outil de la réussite étudiante.

\section{un lieu de sociabilité}

Selon Juliette Lefevre : " La bibliothèque est d'abord un lieu de sociabilité où s'expriment et se retrouvent les différents groupes sociaux et communautés étudiantes. Ce sont les besoins d'échanges entre pairs qui influencent la façon de se placer dans les locaux. Dès lors qu'il y a identification et reconnaissance mutuelle, on cherche à se placer selon le critère d'appartenance pour produire des travaux collectifs ou bien pour s'entraider dans des recherches demandées par le corps enseignant. »

3. Depuis 2013, les étudiants de Lille 3 sont automatiquement inscrits à la bibliothèque par le biais de la carte multiservices.

4. < http://bibliotheques.univ-toulouse.fr/fichiers/etude-emprunt-en-bu-et-reussite-en-licence20121221.pdf >.

5. Juliette Lefevre, Observation des usages et usagers du SCD Lille 3, 6-21 mars 2012, stage d'observation enssib, DCB 21, 2012 (non publié). Ce rapport fait plusieurs fois référence à l'enquête de Mariangela Roselli et Marc Perrenoud, Du lecteur à l'usager : ethnographie d'une bibliothèque universitaire, Toulouse, Presses universitaires du Mirail, 2012 (Socio-logiques). 


\section{Des logiques d'appropriation des écrits}

Elle ajoute : " Le second usage est directement lié à des logiques d'appropriation des écrits. On constate deux types de relation à l'écrit soit par la lecture soit par l'écriture. La relation de production d'écrits semble prépondérante : on recherche la bibliothèque comme le cadre idéal pour écrire. Qu'il s'agisse de remettre en forme les notes de cours, de travailler collectivement un sujet donné, de prendre des notes à partir de livres ou de l'écran de l'ordinateur, les postures liées à l'écrit sont multiples. Elles paraissent primordiales dans l'usage qui est fait du lieu. L'usage des collections comme ressources est présent mais pas central parmi les comportements observés. Cet usage va déterminer la configuration des espaces. »

\section{UN ÉQUIPEMENT OUVERT À TOUS LES PUBLICS ?}

\section{un rayonnement territorial limité}

Il est souvent nécessaire de rappeler que les bibliothèques universitaires sont ouvertes à tous les publics, même si la communauté universitaire est le public naturel et captif. À Lille 3, l'accès et la consultation des ouvrages d'accès libre sont libres et gratuits. Les inscriptions du public autre que le public universitaire (étudiant et enseignant) sont cependant limitées ${ }^{6}$ : $3,5 \%$ des lecteurs actifs, soit 333 usagers sur 9 472. Très logiquement, plus de la moitié de ce public vient de Lille et de ses très proches environs. On compte 42 inscrits habitants de Villeneuve-d'Ascq. C'est trop peu. Un des projets du LC est de participer à la valorisation urbanistique de l'ensemble du quartier et de retrouver ce lien avec la ville, pensé lors de la conception de l'université dans les années 1960. Des collaborations existent avec la médiathèque de Villeneuve-d'Ascq dans le cadre du LC7. D'autre part, Lille Métropole met actuellement en œuvre un projet " Livre et lecture » associant bibliothèques publiques et universitaires.

6. Le public fréquentant la bibliothèque sans effectuer une transaction ne peut être comptabilisé.

7. < http://inforum.univ-lille3.fr/2013/10/le-mois-de-larcheologie-egyptienne-a-lille-3/ >. 


\section{L'impact du Learning center}

Depuis 2011, Lille 3 développe une programmation scientifique du Learning center riche de conférences, d'expositions, d'ateliers à destination des scolaires dans et hors les murs. Les conférences rassemblent régulièrement plus de 100 personnes, le public étant très divers. Les ateliers pour les scolaires sont très demandés ${ }^{8}$. Le Vase qui parle, exposition de préfiguration du LC, présentée à la BU entre avril et juin 2013, a permis d'accueillir plus de 1000 visiteurs, dont parfois un public familial le samedi matin. C'est un public tout à fait inhabituel que la BU a vu arriver dans ses locaux.

\section{PROPOSER DIVERS TYPES D'ESPACES ORIENTÉS PAR DESTINATION}

Le bâtiment actuel est devenu inadapté aux contraintes de fonctionnement des services et surtout aux comportements du public. Les conditions de travail des usagers ne sont pas bonnes, parfois même qualifiées de difficiles (manque de lumière, de confort, de prises, bruit).La configuration du bâtiment rend l'ensemble des services très peu lisible pour les usagers ; elle peut décourager la fréquentation et, dans certains cas, contribuer à susciter des conflits, les usages possibles et prescrits entrant en contradiction avec les pratiques effectives du public.

Dans les conditions actuelles, l'une des principales limites à la qualité du service réside dans la nature des places assises, qui ne constituent ni des postes individuels de travail, ni de véritables espaces de travail en groupe. Cette disposition est peu propice tant pour le travail individuel que pour le travail collectif.

\section{IMAGINEZ VOTRE FUTURE BIBLIOTHĖQUE}

Dans la perspective du LC, le SCD a mené en 2012 une enquête auprès des publics et des non-publics. Elle avait pour objectifs de mieux connaître les usages et les attentes concernant les services, l'offre documentaire

8. < http://inforum.univ-lille3.fr/2013/07/plus-de-1400-visiteurs-sont-venus-rencontrer-nos-fabuleux-paysans-gaulois-a-asnapio/ > . 
mais aussi la dimension architecturale et l'aménagement du bâtiment ${ }^{9}$. Cette enquête qualitative, par entretiens et focus-groupes, a utilisé la méthode de démarche d'évaluation post-occupationnelle* (Post-0ccupancy Evaluation*, POE). Chaque étudiant s'est vu remettre un lot de photos de bibliothèques parmi lesquelles il lui a été demandé de choisir. Durant le $2^{\mathrm{e}}$ exercice, il lui a été proposé d'imaginer trois à quatre espaces du futur bâtiment, avec la possibilité d'illustrer chaque espace d'une ou plusieurs photos. Il s'agissait de photos prises à l'intérieur de diverses bibliothèques universitaires (la BULAC à Paris, Robert de Sorbon à Reims, Maastricht, Utrecht et Leyde aux Pays-Bas) et bibliothèques publiques (Les Champs libres à Rennes, Almere, Amsterdam, Lelystad, Rotterdam aux Pays-Bas, Singapour). On constate une forte convergence des besoins exprimés.

La demande concerne des espaces de travail en groupe, des espaces pour le travail seul, des espaces de détente et cafétéria. Des espaces de détente hors cafétéria sont très importants pour les étudiants C'est la mixité travail-détente, table-fauteuil qui est recherchée : "J'aime bien l’idée d'un espace intermédiaire entre travail et repos » dit un étudiant.

\section{Diversification des espaces et présence au public redéfinie}

Le projet architectural du LC doit permettre de concevoir des espaces adaptés à ses missions. La programmation des espaces de travail devra prendre en compte certains principes d'organisation :

- la création de places de travail adaptées aux pratiques de travail : travail en groupe, travail individuel, travaux tutorés, séminaires, ateliers, labs, research commons avec principes à la fois de zonage et de modularité ;

- l'utilisation de ratios de programmation adaptés aux bibliothèques pour garantir la qualité des espaces de travail. Une bibliothèque «pleine » est un lieu où il est impossible de travailler...

9. Anne Morenvillé, "Enquête auprès des publics de la Bibliothèque universitaire centrale de Lille 3 », Insula. 17 avril 2013. [En ligne] : < http://bsa.biblio.univ-lille3.fr/blog/2013/04/enquetepublics-bibliotheque-universitaire-lille-3/ > . 
Dans ce cadre rénové, le rôle des personnels se trouve considérablement modifié. Liées à la mise en place de la RFID, les opérations de transactions se trouvent déportées. La " médiation ambulatoire »* devient une fonction déterminante du rôle des personnels, toutes catégories confondues, ce qui suppose une grande disponibilité et une redéfinition des emplois. Le personnel doit être « visible » partout.

\section{DES HORAIRES D'OUVERTURE ÉLARGIS}

L'ouverture du nouveau bâtiment amènera très logiquement une réflexion sur la question des horaires d'ouverture. Actuellement, la BU est le service qui a l'amplitude horaire la plus importante dans l'université : $59 \mathrm{~h} 30$ d'ouverture par semaine $(8 \mathrm{~h} 30-20 \mathrm{~h}$ du lundi au jeudi, $19 \mathrm{~h}$ le vendredi, $9 \mathrm{~h}-12 \mathrm{~h}$ le samedi). Il est difficile d'augmenter l'ouverture hebdomadaire pour plusieurs raisons : les impératifs du bâtiment, un bâtiment dépassé qui génère des coûts importants de fonctionnement, l'absence de vie sur un campus situé en zone péri-urbaine et sans résidences universitaires et les coûts (en emplois étudiants notamment).

L'environnement doit pouvoir se prêter à des horaires élargis : ouverture du campus, restauration, sécurité, fonctionnement des infrastructures réseau, moyens de transport et vie sur le campus. La faible attractivité du campus, malgré une politique culturelle très active, et de son environnement urbain fait que la présence des usagers potentiels* et réels est conditionnée par le calendrier universitaire. Une ouverture $7 \mathrm{j} / 7$ - 24 h/24 à l'exemple de certains Learning Centres anglo-saxons n'est pas envisagée. Ces dernières années, l'effort s'est porté sur les bibliothèques associées. Grâce à des mesures d'aide de l'université, elles ouvrent $40 \mathrm{~h} /$ semaine en moyenne du lundi au vendredi. $50 \%$ des bibliothèques associées dépassent cette moyenne et certaines ouvrent jusqu'à $50 \mathrm{~h} / \mathrm{semaine.} \mathrm{Une} \mathrm{harmoni-}$ sation des horaires* de l'ensemble de ces bibliothèques est actuellement engagée, associée à une carte documentaire du campus plus lisible.

La BU a aussi travaillé à l'augmentation du volume horaire plutôt sur un allongement dans l'année que dans la journée : augmentation du nombre de jours d'ouverture dans l'année et ouverture en dehors des calendriers 
de fonctionnement normal de l'université : pauses pédagogiques, vacances de Noël, fin août (pré-rentrée) afin de répondre à une demande étudiante.

\section{QUELLES DEMANDES ?}

L'augmentation du volume horaire d'ouverture de la BU est une demande récurrente. À Lille 3, celle-ci semble porter davantage sur une ouverture plus matinale que vespérale et sur une prolongation le samedi après-midi. L'ouverture le dimanche n'est pas évoquée. L'enquête de 2012 a permis de relever quelques demandes, en particulier l'ouverture le matin dès $8 \mathrm{~h} 00$ pour coller aux horaires des transports en commun et éventuellement l'extension des horaires en soirée jusqu'à $21 \mathrm{~h} 00$ ou $22 \mathrm{~h} 00$, au minimum, en période d'examen (comme, par exemple, Les Nocturnes des BU de Paris Descartes). De façon très légitime, l'ouverture du samedi après-midi est demandée, les horaires actuels ne correspondant pas au rythme " physiologique » du public étudiant.

\section{UN LIEU DE VIE LARGEMENT OUVERT}

Il est clair que la création de nouveaux espaces et la refonte du service à l'usager donneront une latitude certaine pour faire évoluer les horaires d'ouverture, dans leur amplitude quotidienne, hebdomadaire et annuelle. Il est évident que l'ouverture d'un nouvel équipement associée à une programmation scientifique et culturelle destinée à tous les publics devra répondre à des impératifs d'ouverture élargie. Les avancées pourront se faire dans plusieurs directions :

- le développement de services physiques et en ligne ouverts plus largement (robot de retour accessible à l'extérieur du bâtiment, commandes d'ouvrages en magasin et réservations en ligne, accès amélioré aux ressources électroniques) ;

- la flexibilité : l'organisation des espaces détermine le nombre d'agents nécessaires pour assurer l'ouverture au public. Une conception des espaces permettant une ouverture du bâtiment et un dispositif de service public modulables en fonction de la période et de l'affluence (débuts d'année, périodes d'examen, 
plages horaires de forte fréquentation), ou en fonction d'actions spécifiques favorisent une large amplitude d'ouverture ;

- la lisibilité des horaires peut être en concertation avec les autres bibliothèques du territoire.

Les décisions dépendront en grande partie des moyens pouvant être engagés. Il faudra mettre en place un système de compensation pour les personnels sans que cela impacte les jours et heures d'affluence du public et le travail interne.

Les bibliothèques doivent désormais proposer plus que de la documentation, un lieu de vie et de formation offrant de meilleures conditions d'accès à l'information. Des horaires élargis et des services mieux adaptés font partie du programme. Au cœur de l'université, le Learning Center devra aussi tenir compte du contexte territorial et des mutations urbaines. 


\section{PARTIE It}

\section{LES OBSTACLES}

\section{À FRANCHIR : QUELQUES PRÉREQUIS}

1. Les temps de la ville : l'évolution des rythmes de vie interpelle l'offre de services publics par Chrystelle Amblard

2. Tempo territorial : la gestion du temps urbain par Chrystelle Amblard

3. La bibliothèque dans la ville : du chrono-urbanisme à la chronobibliothéconomie par Pierre Franqueville

$+++++++++++++++++++++++++++++++++++++++++++++++++++++++++++++++++$

4. Connaître les publics de sa bibliothèque : impressions, intuitions, constats, mesures par Jean Chaguiboff

$+++++++++++++++++++++++++++++++++++++++++++++++++++++++++++++++++$

5. une situation paradoxale et contrastée par Georges Perrin

6. ouvrir le dimanche? Méthodologie pour des négociations réussies par Jean-François Jacques 


\section{1}

LES TEMPS DE LA VILLE :

L'ÉVOLUTION DES RYTHMES

par

DE VIE INTERPELLE L'OFFRE

DE SERVICES PUBLICS

\section{LES GRANDES MUTATIONS TEMPORELLES : QUELQUES REPÈRES}

De grandes mutations sont intervenues depuis l'après-guerre : allongement de l'espérance de vie, diminution du temps de travail, entrée massive des femmes sur le marché du travail, accélération des vitesses, mondialisation, qui ont bouleversé notre rapport au temps et ont amené une grande diversification des rythmes de vie. Ces nouveaux rythmes, ces nouvelles temporalités, interpellent nos services publics au niveau de leur organisation temporelle, source d'accessibilité ou non, mais aussi au niveau de l'offre de services elle-même.

Nous avons gagné au $\mathrm{xx}^{\mathrm{e}}$ siècle 31 ans d'espérance de vie et, le monde évoluant rapidement et étant plus instable, nous rencontrons au cours de nos vies beaucoup plus de ruptures, de changements qu'auparavant. Cela se traduit par des divorces, des réorientations professionnelles, des périodes de chômage, des innovations technologiques, qu'il nous faut savoir accompagner au niveau de nos services publics. Quelle offre de services mettre en place, pour accompagner les personnes dans leur recherche d'emploi, dans leur formation tout au long de la vie, lors d'évolutions familiales? On voit ici qu'un grand nombre de services publics sont concernés : Pôle emploi, le secteur de la formation professionnelle, les médiathèques, les services sociaux, le logement...

L'allongement de nos vies, mais aussi la réduction du temps de travail ont fait émerger un nouveau temps, celui du temps libre, des week-ends, des vacances, de la retraite, où chacun cherche à profiter au maximum des opportunités qui lui sont offertes. Les plages de temps libre étant très différentes d'une personne à l'autre et les manières de les occuper multiples, 
les services publics concernés sont confrontés à une multiplicité d'usages, auxquels il faut savoir répondre.

Avec l'entrée massive des femmes sur le marché du travail, la vie familiale s'est complexifiée avec des parents qui cumulent une double journée et des enfants qui passent beaucoup de temps à l'école. Les familles avec enfants manquent de temps, elles sont donc peu disponibles pour se rendre dans les services publics en dehors du mercredi ou du week-end, pour rendre des livres toutes les trois semaines. Les parents sont aussi demandeurs de services ouverts entre $12 \mathrm{~h}$ et $14 \mathrm{~h}$ proches de leur lieu de travail et de e-services, de services $24 \mathrm{~h} / 24$ (par exemple, boîte aux livres), pour s'organiser, faire les choses quand ils en ont le temps.

Les jeunes font de plus longues études et ont besoin d'avoir accès à des espaces et des ressources pour étudier, ce qui induit des demandes d'ouverture des bibliothèques sur des horaires élargis, d'accès à des ressources en ligne.

Avec l'augmentation des vitesses de transport, on se déplace plus, plus vite, plus loin. Un usager ne sera donc plus seulement attaché au service public situé à proximité de chez lui, mais il profitera de l'ensemble de l'offre qui lui est offerte (par exemple, prendre un livre à un endroit, le rendre à proximité de son travail, participer à une animation dans un autre lieu).

La montée en puissance des technologies de l'information et de la communication permet d'échanger en quelques fractions de seconde les images, les idées, les informations partout dans le monde. Cela transforme profondément tous nos processus sociaux, communicatifs et interpelle nos services publics.

Nous sommes passés dans les années 1970-1980 à une économie mondialisée, une société de service, de consommation, qui fonctionne $24 \mathrm{~h} / 24$, en temps réel, où l'on veut tout, tout de suite. Cela génère dans les villes des demandes pour des horaires de plus en plus élargis (ouverture des commerces, des services publics, le soir, le week-end, 7j/7). Faut-il répondre à ces demandes ? Comment financer ces horaires élargis ? Quelles répercussions pour le personnel?

Enfin, alors qu'auparavant la vie était principalement rurale et locale, rythmée par les saisons, l'horloge de l'usine et la cloche de l'église, aujourd'hui la vie et les activités se concentrent dans des territoires urbains, 
où vivent et cohabitent des populations et des activités avec des rythmes très différenciés et interconnectés au reste du monde.

\section{RYTHMES DE VIE :}

\section{ENCADRÉ LES GRANDES ÉVOLUTIONS EN CHIFFRES}

- +31 ans d'espérance de vie au $\mathrm{xx}^{\mathrm{e}}$ siècle $(+65 \%)$.

- Le temps libre a été multiplié par 4 et représente 400000 heures au cours d'une vie.

- L'âge moyen de fin d'études est passé de 15 ans au début du $\mathrm{xx}^{\mathrm{e}}$ siècle à 21 ans aujourd'hui. La durée des études représente 30000 heures au cours de la vie.
- Le temps de travail rémunéré a été divisé par 2 au $\mathrm{xx}^{\mathrm{e}}$ siècle et il ne représente plus que $10 \%$ de la vie éveillée, soit environ 70000 heures.

- Le taux d'activité des femmes a doublé : $41 \%$ en 1962, $84 \%$ en 2011.

- Sur le plan technologique, la vitesse des transports a augmenté de $102 \%$, celle des communications de $107 \%$ et celle du traitement de l'information de $1010 \%$.

L'ensemble de ces évolutions nous a fait passer très rapidement d'une société industrielle taylorienne, caractérisée par des rythmes massifs et standardisés à une société à mille temps, où il nous faut réinventer une nouvelle organisation temporelle de nos territoires et de nos services. Comment répondre à la diversité des rythmes de vie, à ces nouvelles attentes ? Comment faire cohabiter des rythmes très différents au sein de nos villes ? Quels rythmes collectifs préserver, réinventer ? Faut-il ouvrir les médiathèques, les commerces le dimanche ? Vers quel modèle temporel local aller : slow, $24 \mathrm{~h} / 24$ ?

Depuis une quinzaine d'années, des acteurs réfléchissent aux mutations temporelles et agissent pour faire évoluer l'aménagement temporel des territoires et des services. 


\section{LE FACTEUR TEMPS : QUELLES INFLUENCES POUR LES SERVICES PUBLICS ?}

Les temps d'accès, l'amplitude horaire des services à la population, les rythmes scolaires, les rythmes de travail, structurent la vie sur nos territoires, il est donc important de s'y intéresser au même titre que l'espace. Le temps que l'on met pour se rendre dans un service public, aller au travail, faire des achats conditionne notre pratique du territoire et facilite ou non l'organisation de nos temps quotidiens. La localisation des différents services publics devra donc être faite au regard des temps d'accès et cela de manière équitable sur l'ensemble du territoire. Par ailleurs, leur implantation devra aussi rechercher à conforter les logiques de proximité, les centralités, les pôles de services, sources de gain de temps pour l'habitant.

\section{DES RYTHMES DE VIE DIVERSIFIÉS}

Les usages des services évoluent en fonction des périodes, selon que l'on est en semaine ou en week-end, l'hiver ou l'été, en période scolaire ou en vacances... Les services publics doivent donc tenir compte de ces différentes périodicités et des pratiques afférentes pour définir leur offre.

Les rythmes de vie des habitants et des usagers du territoire (touristes, actifs...), auront aussi une influence sur l'offre des services publics. Entre une commune périurbaine où la majorité de la population sera absente en journée, un quartier où la population est en grande précarité et au foyer, une zone d'activité, une zone touristique, les attentes en matière d'horaires d'ouverture et de services différeront du tout au tout.

Ainsi par exemple pour les médiathèques, les différentes formules (d'abonnement, de prêt) devront tenir compte des rythmes de vie. Par exemple, une personne en recherche d'emploi, en intérim, un touriste, prendra difficilement un abonnement à l'année ; les familles avec enfants auront du mal à libérer du temps pour venir toutes les 3 semaines rendre les livres... Les modalités horaires des services publics doivent donc s'adapter au contexte local et aux rythmes de vie de leurs usagers. 
Enfin, de plus en plus de personnes courant après le temps, souhaitant l'optimiser au mieux, les pouvoirs publics doivent savoir répondre à ces attentes, en informant la population de l'offre existante, en développant les e-services (prolongation des prêts, réservation de documents, médiathèque numérique) ou les services $24 \mathrm{~h} / 24$ (boîte de retour des livres), et en simplifiant les démarches, en développant les guichets uniques.

\section{LES GRANDES RÉFORMES : QUELLES CONSÉQUENCES SUR LES TEMPORALITÉS LOCALES ?}

Quand de grandes réformes modifient profondément les temporalités locales (par exemple la loi sur les 35 heures, les réformes successives des rythmes scolaires, la modification en 2011 du cadencement et des horaires des trains par la SNCF), ou lorsque des réformes ouvrent de nouvelles possibilités temporelles (l'ouverture des commerces le dimanche par exemple), il est important que l'ensemble des acteurs locaux se concerte pour redéfinir les nouveaux rythmes locaux, les évolutions à apporter à l'offre de services, aux organisations du temps de travail, etc.

\section{La réforme des rythmes scolaires de 2013 et 2014 à Montpellier}

Cette réforme a abrogé la semaine de 4 jours et instauré la semaine de 4,5 jours avec le mercredi matin travaillé à l'école primaire et modifié en profondeur les rythmes de vie locaux.

Pour l'agglomération de Montpellier ${ }^{1}$, la réforme a changé les rythmes de vie de 35000 enfants, de $25 \%$ de la population (familles qui ont un enfant en primaire) et de $22 \%$ des actifs occupés qui habitent l'agglomération et ont un enfant en primaire.

Elle a eu un impact sur l'Éducation nationale, l'offre péri et extrascolaire des communes, les associations culturelles et les clubs sportifs, les équipements culturels et sportifs, l'offre de transports, les entreprises, mais aussi, de manière moindre, sur l'ensemble des services à la population et des commerces (par exemple le mercredi matin, on ne va plus chez le médecin).

1. L'agglomération a été la seule en France à offrir à l'ensemble des acteurs locaux un espace de concertation et de co-construction lié à la réforme des rythmes scolaires. 


\section{SCHÉMA LES IMPACTS DE LA RÉFORME SCOLAIRE}

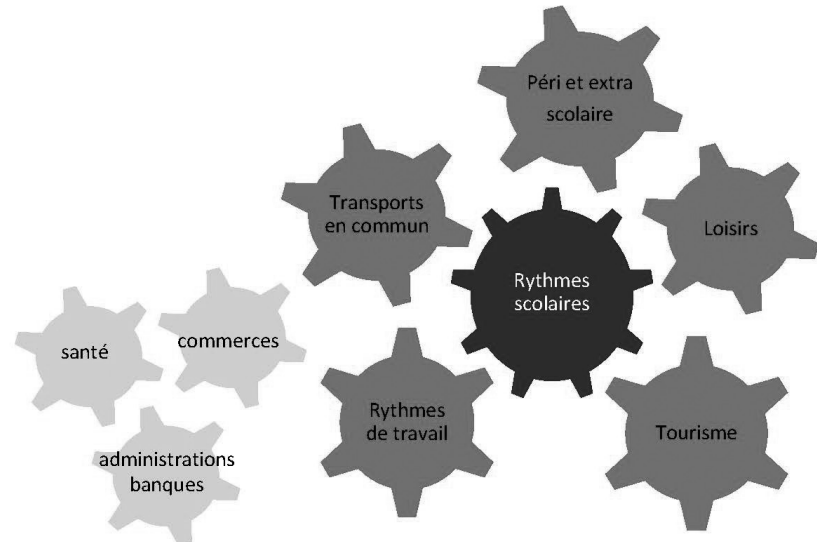

Source : Chrystelle Amblard, 2014.

\section{L'exemple des médiathèques}

Parmi l'ensemble des services impactés par cette réforme, il y avait bien entendu les médiathèques et ce, à plusieurs titres.

Avec la modification des rythmes scolaires (fin des cours plus tôt l'aprèsmidi, mercredi matin travaillé), les sorties scolaires à la médiathèque vont se faire plus rares les après-midi et les familles avec enfants ne fréquenteront plus la médiathèque le mercredi matin. Comment proposer aux classes de nouveaux créneaux horaires les mercredis matins en plus du grand public ? Comment repositionner les animations jeunesses ?

Cette réforme augmente aussi le temps périscolaire et invite l'ensemble des acteurs locaux à se mobiliser pour en faire un temps de qualité. Les médiathèques disposent de nombreuses ressources éducatives, livres, multimédia, jeux, films, musiques, de locaux et de compétences et peuvent être mises à contribution. Cela fait évoluer leur rôle car cette réforme les invite à s'inscrire dans la politique éducative des communes, à accueillir 
des groupes d'enfants sur le temps périscolaire au sein de leurs locaux, à renforcer leurs interventions au sein des écoles (prêt de livres, de jeux, formation des animateurs communaux à la littérature jeunesse et aux jeux, intervention dans les écoles) et à agir en partenariat avec d'autres bibliothèques ou avec des associations comme « Lire et faire lire $»^{2}$.

Comme on le voit avec l'exemple des médiathèques, les mutations temporelles ont des conséquences importantes pour le territoire et ses services. Si les acteurs locaux se concertent et font évoluer leur offre au regard de ces nouvelles temporalités, cela permettra aux services publics de répondre de manière adéquate aux attentes locales. En revanche, si les acteurs locaux ne prennent pas toute la mesure de ces évolutions, leurs offres risquent d'être décalées par rapport aux rythmes de vie, voire inadéquates (par exemple il devient inutile de maintenir les animations grand public pour les 3-10 ans le mercredi matin). Il est donc important d'avoir sur les territoires, des missions temps ${ }^{3}$ à même de solliciter et de travailler avec les acteurs locaux autour de ces évolutions temporelles.

\section{ÉVOLUTION DES RYTHMES DE VIE ET ÉVOLUTION DES SERVICES}

Comme nous l'avons vu précédemment, les rythmes de vie ont beaucoup évolué et le fonctionnement ainsi que l'offre de nombreux services à la population doivent s'adapter.

\section{LES PISCINES}

L'avènement du temps libre et des loisirs, mais aussi l'augmentation de la durée de vie et des préoccupations liées au maintien en forme, font qu'il existe aujourd'hui une forte demande en direction des piscines, au-delà de l'apprentissage de la natation et du sport de haut niveau.

Pour répondre à l'ensemble de ces demandes, les collectivités sont amenées à ouvrir les piscines de plus en plus, sur des plages horaires très larges (par exemple de $8 \mathrm{~h}$ à $22 \mathrm{~h}$ ), 7j/7, mais aussi pendant les vacances.

2. < www.lireetfairelire.org >.

3. Voir la contribution de Chrystelle Amblard : « Tempo territorial : la gestion du temps urbain », p. 73. 
Une fois ces amplitudes d'ouverture définies, il s'agit alors pour la collectivité de répartir les différents créneaux horaires entre les 5 grands types de pratiques que sont le sport, les loisirs, la santé, l'apprentissage de la natation et le sport de haut niveau et les 3 différents types d'occupants que sont le grand public, les scolaires et les clubs sportifs.

Une des briques les plus structurantes de l'organisation des piscines est les rythmes scolaires du primaire, car l'apprentissage de la natation est l'une des compétences obligatoires des collectivités gestionnaires de piscines. Ainsi, quand les rythmes scolaires changent en 2013-2014, l'organisation horaire des piscines est fortement touchée, car avec ces aprèsmidi réduites, les scolaires perdent 4 créneaux horaires sur les 16 dont ils disposent chaque semaine. Les collectivités sont donc amenées à réaffecter 2 créneaux horaires les mercredis matin aux scolaires, alors qu'ils étaient traditionnellement affectés aux écoles de natation des clubs ou au grand public. La modification du mercredi matin induit alors de nouveaux changements sur l'ensemble de la semaine (par exemple, modification des horaires grand public le mercredi après-midi, attribution de créneaux aux clubs le dimanche matin, déplacement des écoles de natation en fin d'après-midi...).

Au-delà de la natation scolaire, d'importantes demandes existent pour des ouvertures grand public, car elles permettent de répondre à des pratiques sportives, de loisirs et de santé, occasionnelles ou régulières, en individuel ou en groupe (couple, famille, amis). Ainsi, les collectivités multiplient les horaires d'ouverture grand public en essayant d'offrir les palettes les plus larges possibles au sein de leurs réseaux de piscines, pour répondre à la diversité des attentes et des rythmes de vie - par exemple la retraitée qui sera libre en semaine, le salarié qui souhaite nager entre $12 \mathrm{~h}$ et $14 \mathrm{~h}$ ou après le travail, la famille ou les jeunes qui voudront profiter de la piscine le mercredi après-midi, le week-end ou pendant les vacances. 


\section{Les horaires élargis des piscines, Montpellier}

L'agglomération de Montpellier a élargi les horaires grand public de ses piscines pour que chacun dispose, à proximité de chez lui, d'au moins une piscine ouverte en nocturne tous les soirs et tout le week-end. Chaque piscine assurant 2 nocturnes en semaine et 2 demi-journées le week-end. Cette réorganisation de l'ensemble des horaires du réseau s'est accompagnée d'une réorganisation des temps de travail et des missions du personnel.

Dans tout projet temporel, il faut rechercher le meilleur compromis entre les attentes des usagers, le fonctionnement des équipements et l'organisation du temps de travail du personnel.

Enfin, il s'agit aussi d'attribuer des créneaux horaires aux clubs qui proposent des activités à l'année à leurs adhérents. Activités variées qui vont de l'école de natation au sport de compétition, à l'activité santé (par exemple l'aquagym) ou de loisirs (par exemple la plongée), qu'il s'agit alors de répartir de manière équitable en répondant aux attentes locales.

\section{L'OFFRE DE GARDE DES ENFANTS DE O À 3 ANS}

L'entrée importante des femmes sur le marché du travail, a amené la France à développer une offre de modes de garde pour les 0-3 ans. Actuellement durant leur temps de travail, les parents ont recours ${ }^{4}$ pour les deux tiers d'entre eux à la garde individualisée (assistante maternelle, nourrice, garde d'enfants à domicile) et pour le tiers restant à la garde collective (crèche, halte-garderie).

L'offre existante répond principalement aux parents qui ont un emploi stable, avec des horaires de travail classiques en semaine (4 ou 5 jours par semaine, du lundi au vendredi, de $8 \mathrm{~h}$ à $18 \mathrm{~h}$ ). Or tous les parents ne sont pas dans cette situation. Un certain nombre est en recherche d'emploi ou en formation, avec des contrats précaires (intérim, CDD), des rythmes de travail atypiques (travail de nuit ou en 3x8, le week-end, horaires variables d'une semaine sur l'autre, temps partiel fragmenté...) et eux aussi

4. Sandrine Micheaux, Olivier Monso, «Faire garder ses enfants pendant son travail ", Insee Première, n¹132, avril 2007. [En ligne] : < http://www.insee.fr/fr/themes/document.asp?reg_ id $=0 \&$ ref_id $=i p 1132 \%$ C2 >. 
ont besoin de modes de garde : de courte durée, du jour au lendemain, à horaires atypiques.

Il est donc nécessaire de faire évoluer l'offre pour qu'elle réponde à la diversité des rythmes de vie actuels et, en particulier, aux parents dans des situations précaires.

Ainsi, il faut développer les capacités de restauration des haltes-garderies de manière à ce qu'elles répondent aux parents en recherche d'emploi ou à temps partiel.

Les crèches collectives ne doivent pas seulement accueillir des enfants 4 ou 5 jours par semaine à l'année, elles doivent aussi pouvoir accueillir des enfants sur des périodes courtes, avec des jours qui varient chaque semaine ou à temps partiel (moins de 4 jours).

Il est important aussi de recenser les assistantes maternelles qui peuvent accueillir des enfants sur des rythmes atypiques, de manière à pouvoir orienter les parents qui ont ce besoin.

Enfin, il est nécessaire d'avoir quelques structures à horaires atypiques pour répondre aux parents qui ont des rythmes atypiques (infirmières qui travaillent $12 \mathrm{~h}$ sur 3 jours par semaine, personnel qui travaille la nuit, le week-end dans les transports, la sécurité, les commerces...) et aux parents en situation de précarité professionnelle (intérim, CDD, stage...).

Quand on réfléchit au modèle horaire des structures de garde, il faut néanmoins toujours garder à l'esprit l'intérêt de l'enfant et de la vie familiale. Généraliser une offre de garde qui répond à la diversité des rythmes de travail, sans se préoccuper des rythmes des enfants n'est pas souhaitable. Dans certaines communes, il y a eu la volonté d'aller vers un élargissement des horaires des structures d'accueil de la petite enfance, qui s'est souvent traduit par des ouvertures jusqu'à $19 \mathrm{~h}$ au lieu des 18 h-18 h 30 habituels. Ces expériences se sont souvent révélées être des échecs car la plupart des parents viennent chercher leurs enfants avant $18 \mathrm{~h}$ 30. En effet, une fois rentrés à la maison, il faut encore s'occuper des toilettes, du repas avant de coucher les enfants vers 20 h-20 h 30. De plus, $19 \mathrm{~h}$ ne répond pas aux vrais rythmes atypiques qui nécessitent des structures ouvertes sur des amplitudes très larges avec des formules extrêmement souples. 
Exemple : «Enfant Présent » une crèche familiale ouverte 24h/24, 7j/7

à Paris accueille beaucoup d'enfants de familles monoparentales dont les parents ont un travail très précaire, avec des horaires atypiques. En offrant un mode de garde adéquat à ces familles, cette crèche permet d'éviter les éventuelles et parfois graves conséquences d'une impossible gestion du temps de vie en famille.

\section{L'ACCUEIL EN BIBLIOTHĖQUE}

Comme on l'a vu plus haut, les médiathèques sont également confrontées à cette nécessité, dictée par la diversité des rythmes actuels, d'offrir à leurs usagers de larges amplitudes horaires à l'échelle de leur réseau, en coordonnant les horaires des différentes médiathèques.

Les nouveaux rythmes scolaires privent, au moins pour une demi-journée, les enfants d'un accueil accompagné familialement. Dans le même temps, l'ouverture du dimanche, comme en témoignent tous les professionnels des médiathèques concernées, constitue un créneau incomparable pour l'accueil des familles, qui ont de moins en moins la possibilité de fréquenter la bibliothèque en semaine. Il ne s'agit nullement d'ouvrir toutes les bibliothèques d'un même réseau ce jour-là, mais de donner une chance aux familles de pouvoir fréquenter ensemble un lieu de culture et de sociabilité dans un climat de détente.

D’autre part, il convient de s'interroger sérieusement sur la fermeture du lundi, qui prive de facto une partie des actifs de la population de médiathèque : les commerçants, qui travaillent le samedi et, pour quelquesuns, le dimanche, et les personnes qui, travaillant les samedi et dimanche (transports, sécurité, santé, etc.), récupèrent du temps de repos et de loisirs ce jour-là.

Ces quelques éléments de réflexion sur la gestion du temps d'accueil du public sont de nature à inciter les collectivités et les professionnels à placer la médiathèque dont ils ont la charge, dans le continuum spatial et temporel du rythme urbain, et à harmoniser leur accueil à la disponibilité temporelle des citoyens. 


\section{2 \\ TEMPO TERRITORIAL : LA GESTION DU TEMPS URBAIN \\ par \\ Chrystelle \\ Amblard}

Initiée à la fin des années 1980 dans quelques villes d'Italie (Modène, Venise, Terni, Sienne, Turin, Catane), la réflexion sur la gestion du temps de la ville et de son harmonisation avec les nouveaux rythmes de vie des citoyens a gagné peu à peu de nombreuses villes en Europe.

En France, cette réflexion s'est concrétisée au sein d'une association créée en 2004 : « Tempo territorial $»^{1}$, qui regroupe les principaux acteurs des politiques temporelles : 20 collectivités territoriales (Paris, Le Grand Lyon, Rennes, Strasbourg, Montpellier Agglomération, Le Grand Poitiers, SaintDenis, Pantin... conseil régional du Nord Pas-de-Calais), des chercheurs, des associations et des professionnels.

Ses missions :

- accompagner les acteurs des territoires dans les démarches temporelles, dans le contexte de transformations et d'évolutions des rythmes de la vie moderne ;

- intégrer la dimension temporelle, à des échelles pertinentes, dans les domaines de l'aménagement et de l'environnement, du développement économique, des transports, de l'action sociale, culturelle, sportive et de loisirs, et des services ;

- construire un centre de ressources et d'innovation sur les enjeux temporels, et favoriser l'échange des bonnes pratiques, en termes de sensibilisation, d'analyse méthodologique, d'organisation de la concertation, de mises en œuvre d'actions concrètes, de communication ; 
- permettre le débat public à l'échelle locale, nationale et européenne sur les pratiques temporelles entre pouvoirs publics, entreprises, habitants, salariés, usagers et leurs représentants.

L'association organise plusieurs fois par an des séminaires et des journées d'étude et d'échange sur des thèmes tels que " temps et services ", " temps et aménagement », " temps des étudiants », etc. Elle fait paraître des publications, par exemple, le Guide pratique sur l'ouverture des médiathèques le dimanche ${ }^{2}$.

2. Le guide pratique sur l'ouverture des médiathèques le dimanche : une méthodologie pour agir, 2011. [En ligne] : < http://tempoterritorial.free.fr/spip.php?article68 >. 


\begin{tabular}{|l|l|}
\multicolumn{1}{c|}{} & $\mathbf{3}$ \\
\hline LA BIBLIOTHĖQUE DANS LA VILLE : & par \\
DU CHRONO-URBANISME À LA & Franqueville \\
CHRONO-BIBLIOTHÉCONOMIE & \\
\cline { 1 - 2 } &
\end{tabular}

\section{L'IRRUPTION DU TEMPS DANS LA CONCEPTION DES VILLES}

"Nous ne construisons pas une bibliothèque pour la ville. Nous construisons la ville ». Cette interpellation de Brian Gambles ${ }^{1}$ résume ce que nous pourrions nommer la "piste de l'urbanité » qui désigne à la fois un périmètre d'observation (les relations que la bibliothèque entretient avec la réalité urbaine), une méthode pour mener à bien cette observation, enfin une méthode pour repenser les équipements du livre, imaginer ce que pourrait être la bibliothèque de demain.

Ce dernier item désigne l'étape de la programmation comme un moment extrêmement important : il ne s'agit plus de faire référence à un ensemble de normes, d'enregistrer les demandes tacites, d'observer ce qui se fait ailleurs, mais d'anticiper le rôle de la bibliothèque dans la cité contemporaine. Il faut alors admettre que la bibliothèque est un outil au service de l'urbanité qui déborde la stricte sphère culturelle et qu'elle doit évoluer en fonction d'un ensemble de facteurs extérieurs, qu'elle est un objet éminemment plastique doté d'une grande capacité d'adaptation.

Déclarer que la bibliothèque est au service de l'urbanité, c'est affirmer que l'on peut favoriser cette adaptation, voire l'accélérer. L'urbanité est une des valeurs du lointain de la bibliothèque : elle permet de prendre du champ, d'ouvrir la réflexion.

Dans cette perspective, la thématique de l'accessibilité permet de réinterpréter cette question au regard des avancées de ce que François Ascher, théoricien et praticien de la ville, nommait le chrono-urbanisme, ce dernier étant défini comme la capacité à intégrer la dimension temporelle

1. Brian Gambles est directeur-adjoint de la Bibliothèque de Birmingham. 
dans l'aménagement urbain. Ce dernier est l'auteur d'un article fondateur paru en $1997^{2}$. Il y évoque les profondes mutations à l'œuvre entre la ville industrielle classique et la ville contemporaine. Différents ordres (économique, institutionnel, familial et religieux) s'ordonnent pour former une configuration temporelle correspondant à celle de la ville industrielle classique : l'économique prime sur les autres, l'institutionnel et le religieux ne jouent plus qu'un rôle régulateur, le familial est borné en mode mineur par les trois autres. Les différentes activités sont segmentées autour de l'activité principale : le travail. L'activité de loisir vient se loger dans un temps résiduel. À cette temporalité est associé l'urbanisme fonctionnel qui sépare les espaces du travail de ceux de l'habitat.

Aujourd'hui, la configuration temporelle de la ville est profondément modifiée : le temps travaillé des citadins a diminué, mais si le travail reste premier par ses effets structurants, il s'est aussi assoupli, individualisé et correspond désormais à des rythmes horaires diversifiés et changeants. Les ordres intermédiaires religieux et institutionnels ne sont plus régulateurs et ordonnateurs de pratiques collectives fortes. Surtout, l'ordre familial s'est profondément disloqué en un ensemble de pratiques individuelles qui appellent des solutions où le singulier prime sur le collectif. Le loisir émerge comme un nouvel ordre à part entière. Et surtout, la structure temporelle n'est plus segmentée : l'individu contemporain fait plusieurs choses à la fois ou dans une alternance plus rapide, plus concise, plus imbriquée. L'individu contemporain ne connaît plus la vacance, le temps libéré de toute activité. Au mieux, il ralentit son activité. Progressivement, l'organisation urbaine apprend à répondre à cette exigence grandissante : la ville à la carte, 364 jours sur 364, 24h/24 !

C'est également un ensemble de systèmes techniques qui sont mis à sa disposition, lui offrant une parfaite maîtrise de l'espace et du temps. Nous n'en citerons ici que deux pour leur proximité avec notre sujet:

- le véhicule personnel associé à un terminal de communication conjuguant radio, téléphone, messagerie, GPS. Il a permis de

2. François Ascher, « Du vivre à temps au chrono-urbanisme », Les annales de la recherche urbaine, $\mathrm{n}^{\circ} 77,1997$. 
transformer le temps non productif du transport et de la mobilité en un temps foisonnant rattaché à d'autres sphères d'activités : professionnelles, familiales, de loisir, de formation...;

- le multimédia qui fournit à l'utilisateur un outil multifonctionnel qui, grâce à Internet, donc sans limitation de distance et d'horaire, permet d'accéder de façon désynchronisée aux réseaux d'informations mondiaux, de consulter en direct ou en différé des produits numériques constitués de textes, d'images fixes, animées, de sons et musiques, etc., de les retraiter, de les stocker. Par ailleurs, un ensemble d'outils logiciels individualisés rendent potentiellement caduque la fixation d'un poste de travail ou d'étude à un lieu précis : bureau, entreprise, université, domicile.

Libéré de l'entrave du temps et de l'espace, le citadin contemporain, s'il semble acquérir une relative autonomie, devient également dépendant d'un système technico-économique lui assurant une continuité de fonctionnement des différents services et terminaux numériques dont il fait usage.

De fait, la ville devient la matrice quotidienne où se jouent en permanence ces multiples ajustements d'intérêts divergents. Les débats sur l'ouverture des services publics et des enseignes privées le dimanche et les jours fériés, sur la nécessité de préserver une vie nocturne tout en respectant le calme des riverains, sur les demandes des entreprises de fret d'utiliser les aéroports la nuit, ne sont que les symptômes des profondes modifications sociétales en cours.

Mais la ville est également le lieu où des solutions s'inventent pour apporter des réponses aux nouvelles exigences des citadins en matière d'accessibilité (temporalité et mobilité). Solutions qui prennent la forme d'espaces publics, d'équipements ou de services. À cet égard, la bibliothèque est une institution remarquable parce qu'elle est un espace public, gratuit et libre d'accès, doté d'un ensemble de services offerts à tous les citoyens sans exception. Mais la question n'est pas uniquement de savoir comment la bibliothèque peut être plus accessible à ces visiteurs. Elle revient plutôt à se demander si la bibliothèque peut jouer un rôle dans les solutions à apporter ? Pourquoi et comment ? L'objet de cette contribution 
est de lancer la discussion pour qu'elle soit reprise en d'autres lieux, avec d'autres moyens théoriques et pratiques d'investigation.

\section{LA BIBLIOTHĖQUe, PLASTICITÉ ET TENSIONS}

Si l'on résume les tendances des bibliothèques publiques actuelles, que constate-t-on?

C'est tout d'abord le rapport à l'espace clos du magasin et de son stock, qui a été bouleversé. La bibliothèque est aujourd'hui connectée à l'univers infini du savoir sous ses formes les plus diverses lorsqu'Internet a permis de relier les bibliothèques entre elles ainsi qu'au savoir diffus de la blogosphère. Plus encore, la bibliothèque accède à un statut d'acteur lié à sa capacité de créer de l'information discriminante : la bibliothèque c'est le monde ramassé en peu d'espace et en peu de temps, ce qui le rend de fait plus accessible.

Mais, en deçà de cet infini, la bibliothèque c'est aussi une capacité à s'inscrire dans le local. La bibliothèque détient, pour qui sait l'écrire et le lire, le récit de la vie des entreprises, des institutions, des acteurs. Elle inscrit son action dans une perspective de développement territorial.

La bibliothèque, c'est l'économie de la consultation qui s'installe progressivement face à une économie de la conservation. De fait, la bibliothèque devient média offrant accès aux autres médias qui constituent ses fonds. La bibliothèque devient alors outil de médiation, service qui appelle une compétence d'éditorialiste puisqu'il faut distinguer la bibliothèque du distributeur automatique de livres. Ainsi, le centre de gravité du métier se déplace de la gestion du fonds vers une offre « servicielle » (à l'instar du Guichet du savoir généré par la Bibliothèque municipale de Lyon) centrée sur la médiation humaine et la satisfaction d'un usager dont le profil s'éloigne du lecteur traditionnel pour devenir un utilisateur caméléon, fantasque et versatile multipliant ses motifs et ses usages lors d'une même visite de la bibliothèque.

Mais la bibliothèque c'est aussi un lieu dans l'espace urbain, espace clos et protecteur qui accueille le corps, le temps d'un instant, quand la ville se fait flux et modalités de déplacements, quand le savoir se fait flux continu d'informations dématérialisées. La bibliothèque est abri temporel autant que spatial de l'individu et du collectif, lieu du séjour prolongé et 
confortable. Ainsi, la bibliothèque devient le lieu d'accueil de multiples temporalités d'usages, parfois divergentes.

Mais la bibliothèque devenant ainsi une interface matérielle propre à faciliter l'accès aux informations numériques dématérialisées (textes, images fixes et animées, son), elle doit inventer une architecture de la consultation numérique capable d'accueillir les multiples interfaces disponibles en tenant compte des exigences de confort adaptées à différentes temporalités d'usages. Ceci est rarement le cas dans les projets classiquement proposés par les architectes de bibliothèques, qui réduisent leurs efforts à l'invention d'une boîte plus ou moins séduisante.

\section{TROIS BIBLIOTHĖQUES EN UNE}

On peut donc conclure qu'au regard du problème de l'accessibilité il y a sans doute au moins trois bibliothèques en une :

- la bibliothèque dématérialisée, ouverte $24 \mathrm{~h} / 24,7 \mathrm{j} / 7$, qui n'est encore qu'une ébauche de ce qu'il serait possible de faire au regard des catalogues potentiellement en jeu ;

- la bibliothèque servicielle, à peine ébauchée elle aussi, qui vient se loger pour l'instant dans le lieu bibliothèque et qui hérite de ses contraintes d'accessibilité, mais qui pourrait s'en affranchir. Elle est un gisement de services mais aussi de métiers pour le bibliothécaire de demain, dont le potentiel de développement est important au regard des mutations urbaines en cours ;

- le lieu bibliothèque, dont la moyenne d'ouverture en France n'est que de 20 heures par semaine et qui subit, plus qu'il ne préfigure, l'exigence des multiples temporalités d'usage que nous avons évoqué.

La complexité vient du fait que le lieu bibliothèque entretient des relations étroites avec la bibliothèque numérique dématérialisée et avec la bibliothèque servicielle, des relations encore mal connues et donc mal exploitées. La bibliothèque numérique est encore si peu aboutie en France qu'il est difficile de pointer ses caractéristiques, ses atouts et ses handicaps au 
regard du problème de l'accessibilité. La révolution structurelle produite par Internet débouche sur une organisation moins dépendante des temporalités et des distances. La révolution Internet permet au grand guichet du savoir qu'est la bibliothèque numérique de méconnaître et les distances et les fractionnements rendus obligatoires par les organisations du travail. Le bibliothécaire numérique ne connaît pas la vacance.

La bibliothèque servicielle constitue le terrain sur lequel l'innovation d'accessibilité présente le plus de potentialités. Nous entendons par bibliothèque servicielle l'ensemble des prestations susceptibles d'être offertes aux visiteurs de la bibliothèque. Actuellement, les prestations offertes sont quasi toutes liées à la mise à disposition du fonds, à l'aide à la recherche, aux actions de médiation portées par la bibliothèque dans et hors ses murs, au soutien à l'accessibilité pour des publics empêchés. C'est déjà beaucoup, c'est encore peu par rapport au gisement d'usages potentiels pouvant être générés par la bibliothèque de demain.

À cet égard, les services " questions-réponses », tel le "Guichet du savoir » de la Bibliothèque municipale de Lyon ${ }^{3}$, sont emblématiques des nouveaux services offerts aux usagers. Il s'agit là d'une accessibilité aux collections d'un type nouveau, avant tout cognitive, sans détour par le catalogue, sans limitation d'espace ou de temps et sans obligation d'inscription préalable à la bibliothèque.

Cette innovation constitue l'archétype d'une démarche servicielle. Une telle démarche est extensible à d'autres domaines d'intervention et dont la bibliothèque pourrait s'emparer, tels :

- la gestion et la médiation des données numériques ;

- la gestion de la contribution publique et, plus globalement encore, l'organisation du débat public sur les sujets contemporains ;

- la valorisation des données territoriales, leur retraitement en vue d'une communication aux acteurs économiques, sociaux, éducatifs, sans compter la valorisation territoriale ;

3. À l'instar de ce qui a été prévu dans le cadre de la rénovation de la Bibliothèque de la PartDieu, qui mettra à la disposition de ses visiteurs lève-tôt et couche-tard des espaces dédiés qui fonctionneront en dehors des heures d'ouverture de l'équipement principal. 
- la construction d'un récit territorial fondée sur la réunion, la valorisation et la médiation des données émanant du territoire...

L'ensemble de ces domaines a besoin d'un opérateur qui traite (éditorialise) et rende accessible (diffuse) une information, un savoir, un récit, une parole disséminée, en s'appliquant à répondre à des usagers (service), sur place et à distance, dans des délais et des modalités suffisants pour leur permettre de prendre des décisions ou d'agir opportunément (pertinence). Le lieu bibliothèque ne sort pas indemne de ces grands bouleversements. Le diagnostic est à chaque fois similaire : emprunteurs en baisse, visiteurs en hausse impliquant une plus grande attention à leurs demandes singulières, multiples et foisonnantes. Les maîtres d'ouvrage publics se voient aujourd'hui contraints de repenser les accès et les circulations, notamment les halls où se concentrent souvent les signes d'une vétusté prématurée, l'architecture et les aménagements intérieurs, les mobiliers de consultation et de présentation des collections, les interfaces numériques encore trop peu importantes en nombre, les outils de médiation, l'ensemble relevant d'une époque où le visiteur était encore le sage lecteur discipliné.

La bibliothèque numérique et la bibliothèque servicielle imposent au " lieu bibliothèque » une exigence d'accessibilité temporelle, physique (urbaine, architecturale, fonctionnelle), ainsi que d'accessibilité cognitive qui constitue sans doute le point sur lequel la marge d'évolution est selon nous la plus grande.

Ces trois bibliothèques vivent et se développent sur des temporalités et des degrés différents d'accessibilité. Aussi, l'usager qui prend l'habitude d'utiliser la bibliothèque numérique, ou tout simplement Internet et les immenses services et applications offerts, ne comprend pas pourquoi les deux autres bibliothèques ne sont pas à sa disposition hic et nunc. Car la bibliothèque numérique s'est affranchie des temporalités du travail humain. Il est possible de requérir ses services à n'importe quel moment du jour ou de la nuit, 7j/7. D'où l'on voit germer à juste titre des revendications pour l'ouverture plus importante des bibliothèques.

Mais le lieu bibliothèque présente encore une autre forme de limitation : sa difficulté à accueillir encore parfaitement les postures individuelles ou collectives liées à leur consultation. De ce fait, c'est sans doute toute la 
chaîne de fabrication de la bibliothèque qui est à repenser, la nature des compétences également qui sont appelées à collaborer. Est-ce toujours à l'architecte d'assumer ce rôle de chef d'orchestre quand on sait que c'est la relation aux collections qui est désormais centrale et que les maîtres d'œuvre sélectionnés sont essentiellement préoccupés à construire une enveloppe et ses aménagements sur la base d'une conception de l'espace architectural pré-numérique. On peut également poser la question de la responsabilité de celui qui assume l'élaboration du programme architectural, où la question de la description des usages (numériques et non numériques) devrait être centrale alors qu'elle n'est qu'esquissée.

\section{COMMENT REPENSER L'ACCESSIBILITÉ AU REGARD DE CES TENSIONS ET INSATISFACTIONS?}

Cette triple distinction est éclairante car les oppositions mais aussi les complémentarités qu'elle révèle sont intéressantes au regard de notre sujet :

- la bibliothèque numérique, c'est l'utopie d'une accessibilité temporelle sans limite, mais c'est aussi la bibliothèque absente, désincarnée, qui retire à la bibliothèque sa fonction essentielle d'espace public ;

- la bibliothèque servicielle, c'est en premier lieu la bibliothèque en acte, le cœur du métier, le service consistant à valider la pertinence de l'information remise. Mais bientôt cette bibliothèque s'emparera, si elle le souhaite, d'autres fonctions urbaines liées à la maîtrise des informations, à l'invention et à la diffusion des récits territoriaux dont la cité d'aujourd'hui a éminemment besoin ;

- le lieu bibliothèque, c'est l'infrastructure la plus soumise aux contraintes économiques, et la plus fragile dans l'économie globale du système : elle pourrait bien disparaître si elle n'arrivait plus à faire la preuve de sa nécessité. C'est aussi la part la plus délicate à mettre en œuvre car elle doit en permanence évoluer pour accueillir les deux autres dans leurs transmutations permanentes. 
Pour repenser dans ces jeux singuliers d'oppositions et de complémentarités l'accessibilité de la bibliothèque, trois pistes peuvent être esquissées que l'on présentera dans une progressivité de la torsion que l'on appliquera au modèle actuel.

\section{AMÉLIORER L'EFFICIENCE DE LA BIBLIOTHĖQUE AVEC LES MOYENS ACTUELS EN FONCTIONNEMENT}

Faciliter l'accessibilité c'est repenser le couple temporalité/mobilité. Cela impose de positionner le lieu bibliothèque sur des points de connexion, des hubs urbains, sur les trajectoires naturelles de ses publics potentiels. Cela impose de ne pas négliger la réflexion urbaine, d'accentuer les démarches intercommunales et de ne plus raisonner en communes-propriétaires, en jouant la carte des parcours ou en venant loger la bibliothèque sur de nouveaux nœuds de centralité.

$\mathrm{Au}$ placement de la bibliothèque sur les nœuds de connexion pourrait s'ajouter une proposition plus prospective de services mobiles pour accompagner le visiteur dans ses déplacements en s'appuyant sur l'ingénierie des concessionnaires chargés de transporter les voyageurs quotidiens de nos cités.

Cela passe aussi par la conception de programmes d'équipements qui intègrent des espaces différenciés capables de fonctionner en autonomie temporelle par rapport au reste de la bibliothèque.

Mais surtout, pour profiter du passage d'une économie de la conservation à une économie de la consultation, d'une économie de l'emprunt à une économie de la médiation, faciliter l'accessibilité implique de repenser l'organisation et la distribution des tâches (à moyens égaux) en utilisant les outils technologiques actuels (RFID) facilitant l'autonomie des usagers pour les opérations de prêt et de retour. 


\section{MUTUALISER LES MOYENS (LIEUX ET PERSONNELS), SURTOUT EN TERRITOIRE RURAL, POUR PARTAGER LES CHARGES DE FONCTIONNEMENT}

Pour réduire les coûts du service public, on peut repenser l'économie et l'usage des lieux dédiés en les transformant en lieux mutualisés accueillant un bouquet de services culturels et non culturels, publics mais aussi privés, comme cela se fait déjà dans certaines petites communes. Les économies réalisées peuvent être mises à profit pour dégager une accessibilité horaire supplémentaire. Un pas de plus peut être franchi en mutualisant les équipes. On évoquera ici l'exemple de la Poste qui envisage pour compenser la baisse inexorable du courrier, de transformer les 90000 postiers en acteurs des services émergents de proximité. La réflexion est intéressante : son point de départ consiste à valoriser le cœur du métier et les qualités reconnues du postier : la proximité quotidienne avec le citoyen et le fait que le facteur inspire la confiance du public.

\section{Élargir l'objet social de la bibliothèque pour convaincre les décideurs d'accroître ses moyens en fonctionnement}

Pour être dotée de moyens supplémentaires, la bibliothèque doit convaincre qu'elle recèle des potentialités, des gisements de services nouveaux qui servent l'urbanité mieux qu'aucun autre équipement public. Faire que son « objet social » (l'ensemble des services que la bibliothèque fournirait) soit un argument irréfutable à une ouverture plus large. Ceci passe par la mise en avant du rôle d'espace public du lieu bibliothèque, mais aussi par l'accroissement de son offre servicielle autour de son métier de base. Deux directions qui la désigneraient comme un acteur incontournable de la vie collective.

Qu'il soit nécessaire de pétitionner ${ }^{4}$ afin de demander des moyens pour que les bibliothèques soient plus largement accessibles, on ne saurait prétendre le contraire. Nous serions cependant tentés d'interpréter cette initiative comme le signe de l'épuisement d'un modèle qui a désormais

4. Voir la pétition de l'association "Bibliothèques sans frontières ». [En ligne] : < www.bibliosansfrontieres.org/index.php?option=com_k2\&view=item\&id=354:ouvrons-les-biblio\&Itemid=55 >. 
du mal à convaincre élus et décideurs qu'il est nécessaire de le doter en moyens nouveaux. N'y a-t-il pas, au-delà de cette légitime question des moyens, besoin d'une réforme de fond qui questionnerait le périmètre d'action que l'on traduirait par " l'ensemble des services rendus » par l'entreprise Bibliothèque et de sa place dans le faisceau des services de la cité contemporaine - quitte à voir s'éloigner la bibliothèque de la sphère strictement culturelle ? Mais n'est-ce pas déjà le cas ? 
4

CONNAÎTRE LES PUBLICS DE SA BIBLIOTHĖQUE : IMPRESSIONS, INTUITIONS, CONSTATS, MESURES

par

Jean

Chaguiboff

\section{EXPLICITER CE QUE L'ON SAIT ET L'UTILISER DANS SES CHOIX}

$++++++++++++++++++++++++++++++++++++++++++++++++++++++++++++++++++$

Au moment où le responsable de bibliothèque veut définir un projet, décider de l'orientation des actions à initier, la prise en compte des réalités de son public représente un enjeu fort. Il ne doit négliger aucun des éléments qui sont portés à sa connaissance, aussi bien les données tangibles, mesurables et mesurées, que les remontées plus « qualitatives » des usagers. Il doit continuer à accorder une large place à l'image que ses collaborateurs ont du public, et, au bout du compte, à sa propre intuition.

Le responsable d'une bibliothèque a nécessairement une vision du public qui la fréquente. Mais cette connaissance peut être plus ou moins approfondie, plus ou moins exploitée dans l'élaboration de nouvelles offres de la part de l'établissement. L'objet de ces quelques pages est de renforcer la réflexion sur la façon dont la connaissance du public peut se structurer et renforcer la pertinence de décisions d'aménagement ou d'organisation.

\section{OBSERVER LES FLUX : QUI VIENT ? À QUELS MOMENTS ? QUI FAIT QUDI ?}

La connaissance du public se construit d'abord tout naturellement à partir de l'observation des flux et des comportements. Qui vient ? À quels moments ? Qui fait quoi ? Les échanges avec les usagers, de simples remarques, ou des conversations plus prolongées apportent un éclairage sur le ressenti et les attentes exprimées. Les retours viennent également des équipes elles-mêmes, témoins quotidiens des réactions et commentaires des usagers.

On note naturellement une forte influence de certaines remontées spontanées des usagers. Celles-ci sont souvent liées à des insatisfactions ou 
des attentes déçues, plus rarement à des surprises heureuses. Les réclamations sont plus faciles à exprimer que les compliments, même si, fort heureusement, ceux-ci peuvent émerger à la faveur d'une modification, d'un nouvel aménagement. Les critiques directement formulées sont à considérer sérieusement et peuvent ouvrir les yeux sur telle ou telle défaillance de la bibliothèque. Mais elles risquent aussi de ne toucher qu'à certains aspects très ponctuels ou conjoncturels, ou ne concerner qu'une fraction très limitée du public.

Il y a toujours des changements qui peuvent faciliter la vie des usagers ou accroître leur satisfaction bien que ceux-ci n'aient jamais pensé émettre des critiques ou des souhaits en ce sens.

Parmi les données mesurables dont peuvent disposer les bibliothèques on compte les statistiques sur les prêts, sur le nombre d'entrées par journée ou par plage horaire. Des enquêtes auprès du public peuvent être organisées. Celles-ci permettent d'aller plus loin dans la connaissance des habitudes et des motivations. Cela demeure cependant des opérations lourdes qui doivent être réalisées selon les règles de l'art pour être valides. À cet égard, certains ouvrages récemment publiés peuvent constituer de précieux outils ${ }^{1}$.

Aussi, pour agir dans le bon sens, il faut s'arrêter. Cette idée du public qui se construit à partir d'une multiplicité de faits et de témoignages, peut se révéler trop confuse pour être utile. Il faut prendre du recul, se donner un temps pour la réflexion, hors de toute autre contrainte externe pour déterminer ce qu'est la nature des usagers de la bibliothèque, quel service il convient d'apporter aux uns et aux autres, et comment évoluer vers une meilleure adaptation, vers plus d'usages pour plus d'usagers.

Cela conduit à structurer la connaissance qu'on a du public pour en tirer des directions opérationnelles. Les pages qui suivent ont l'ambition d'apporter des éléments utiles à ce travail.

1. Entre autres: Christophe Evans (dir.), Mener l'enquête. Guide des études de public en bibliothèque, Villeurbanne, Presse de l'enssib, 2011 (coll. La Boîte à outils ; 22). 


\section{DÉFINIR LES PUBLICS DE RÉFÉRENCE}

Le public qui séjourne à la bibliothèque n'est pas forcément représentatif de la totalité du public de la bibliothèque. Dans nombre de bibliothèques, en effet, les responsables constatent aisément que les usagers qu'on rencontre dans les salles à un moment donné ne sont pas représentatifs des usagers qui défilent à l'entrée au cours de la journée. Pourquoi ? Parce qu'une partie des usagers ne fait que passer rapidement, pour rendre et emprunter des ouvrages alors que d'autres s'installent pour des heures, voire pour la journée, qui pour lire, qui pour étudier, qui pour rechercher un emploi sur Internet.

De fait, la structure du public, suivant qu'on l'étudie en sollicitant les personnes dans les salles, ou en les interrogeant à la sortie, ne sera pas semblable, même en utilisant dans les deux cas une technique identique, celle dite de "tirage aléatoire ». Dans les salles, on trouvera un plus grand nombre de jeunes, de chômeurs et de retraités que dans l'enquête en sortie de bibliothèque où la proportion d'actifs et de mères de famille sera plus élevée.

L'explication est simple : en sortie, la probabilité d'être comptabilisé est la même pour tous les usagers, dans les salles cette probabilité est différente : elle est d'autant plus forte que l'usager demeure longtemps.

Il s'agit pourtant bel et bien du même public. Comment distinguer et éviter l'ambiguïté du terme "public »? Il faut introduire ici une notion essentielle en statistique qui est celle de la «population de référence ».

On comprend ici que selon chaque projet envisagé, la population de référence ne devra pas être la même. En effet, si on réfléchit sur le réaménagement des espaces, il est logique de considérer en priorité la population des usagers présents dans les salles. Si la préoccupation est celle du déploiement des collections, c'est l'ensemble des usagers entrants/sortants qui est concerné. 
De ce fait, le choix de la méthode d'investigation est déterminant ainsi que sa constance. À titre d'exemple, dans une bibliothèque, une enquête auprès du public des inscrits avait été menée à quelques années d'intervalle, mais avec des procédures différentes : interrogation téléphonique à partir du fichier lors de la première phase d'enquête, puis directement dans les salles pour la seconde. Les résultats semblaient montrer que les inscrits venaient plus souvent. En réalité, une telle différence était avant tout imputable au changement de méthode. Il est logique de ne pas ou peu rencontrer les inscrits qui viennent rarement, alors que, dans le cas du répertoire des inscrits, ces derniers ont exactement la même probabilité que les autres d'être interrogés. De là l'impression probablement erronée d'une augmentation de la fréquentation des inscrits entre les deux phases d'enquête.

Ici aussi, le choix de la population de référence dépend des priorités retenues par les responsables : satisfaire davantage les inscrits qui viennent souvent, ou tenter de séduire aussi ceux qui se font plus rares.

On entend parfois dire que les usagers fortement engagés dans la vie active peuvent "se débrouiller » pour aller en bibliothèque aux heures et jours ouvrables. Cela peut être vrai en tout ou en partie. "Ceux qui le veulent le peuvent ». Cette conception est sous-tendue par une prise en considération de la population de référence de l'ensemble des usagers. Aussi, on peut se dire qu'un réaménagement des horaires n'aurait pas d'effet notable sur la structure du public de la bibliothèque, si effectivement chacun peut y avoir accès, même dans des conditions difficiles d'accès ou de confort.

En revanche, en changeant de point de vue, en se penchant cette foisci non plus sur le public en général, mais sur la population des usagers présents dans les salles, on comprend qu'une facilitation pouvant amener les personnes à venir plus souvent, à rester plus longtemps, sera de nature à changer la structure de cette population. Ceux qui venaient rapidement et rarement à cause de leurs contraintes apparaîtront en plus grand nombre au sein de cette population. Un nouveau public peut certes être attiré par de nouveaux horaires, mais même si cela n'était pas le cas, 
la fréquentation et la composition de la population des usagers venant à la bibliothèque ont des chances d'en être modifiés.

Un aménagement des horaires peut conduire à un accroissement de la fréquentation de la bibliothèque sans conduire nécessairement à une augmentation du nombre des usagers.

Il existe cependant une partie du public de la bibliothèque qui constitue une zone d'ombre : il s'agit des usagers " potentiels », des personnes qui par leurs intérêts, leur zone d'habitation ou d'activité, pourraient bénéficier des services de la bibliothèque mais qui pour des raisons variées, horaires ou déplacements malcommodes, mauvaise information, manque d'incitation, n'y viennent pas. Cette population constitue tout naturellement une cible. Elle est malheureusement plus difficile à connaître, si ce n'est au travers des déclarations de quelques nouveaux usagers qui se sont trouvés dans cette situation.

\section{DU « BESOIN » À LA MISE EN PLACE DU SERVICE, LE CHEMIN À SUIVRe}

Besoins, envies, opportunités, contraintes... Le rapport à la bibliothèque varie d'un usager à l'autre, d'un moment à l'autre. Le souci d'adapter l'offre de la bibliothèque aux besoins des usagers est un souci légitime. Il convient cependant ici de revenir sur le mot « besoin » pour en bien préciser le sens.

Les jeunes qu'on met en contact avec les livres et qui deviendront plus tard des lecteurs assidus n'ont pas généralement au départ un fort " besoin » de lecture. Les familles qui peuvent se livrer à toutes sortes d'activités le dimanche n'ont pas nécessairement " besoin » d'aller en bibliothèque ce jour-là. Qui peut dire cependant qu'elles ne préféreraient pas cette sortie à d'autres si cette possibilité leur était ouverte?

Il y a effectivement " besoin » lorsque les alternatives n'existent pas ou sont insuffisantes. Les étudiants qui manquent d'espace de travail, les 
lecteurs à budget restreint qui ne peuvent pas s'acheter des livres autant qu'ils le voudraient, entre autres.

Mais qui s'avancerait à dire que les bibliothèques sont consacrées exclusivement aux usagers qui en ont véritablement « besoin » ? Le public des bibliothèques va bien au-delà : il manifeste des envies, des intérêts, des curiosités. Son attachement à la bibliothèque peut se fonder sur le plaisir, la commodité, l'habitude, plus que le " besoin ». (On distingue ici bien sûr le " besoin » d'une bibliothèque qui répond à une nécessité sociale, besoin d'une communauté, et les motivations de chacun des individus qui composent cette communauté).

D'autres activités entrent en concurrence avec la visite en bibliothèque : aller à la piscine, au cinéma, partir en promenade ou jouer sur une console de jeux. Il y a des moments où on pourrait aller en bibliothèque, mais si elle est fermée, on fait autre chose.

L'usager a toujours le choix. L'ouverture élargie de la bibliothèque peut ne pas répondre à un « besoin » ressenti, mais elle vient enrichir le champ des possibles pour les citoyens.

Une tentation fréquente chez les commanditaires d'enquête est de faire demander aux personnes constituant un public quels sont leurs besoins. La démarche donne souvent lieu au recueil d'informations triviales qui vont dans le sens de " plus de » par rapport à l'existant. Il est en effet difficile pour une personne interrogée d'exprimer le besoin de quelque chose qu'elle a du mal à définir, ou qu'elle conçoit mal. À titre d'exemple, personne n'avait « besoin » de smartphone avant que ceux-ci ne soient diffusés dans le commerce.

Une offre nouvelle de la bibliothèque peut être parfaitement pertinente et couronnée de succès sans forcément répondre à ce qu'on a coutume d'appeler un " besoin ». Cette offre doit simplement être adaptée à un public qui a ce qu'on appellera « une disposition à » en profiter, ceux dont les intérêts, les modes et rythme de vie coïncident avec les avantages qu'apporte la nouveauté. Cela va bien au-delà de ceux qui en auraient ouvertement « besoin ». 


\section{ouvrir le dimanche : un progrès qualitatif}

Le public d'une bibliothèque a-t-il besoin qu'elle soit ouverte le dimanche? En majorité, peut-être pas. Mais si la possibilité s'en présente, nombre d'usagers ne vont-ils pas changer leurs habitudes, en venant différemment : à d'autres moments, plus souvent, plus longtemps, ou accompagnés de leurs proches ? Sans parler nécessairement de réponse à un besoin, on pourrait alors parler de progrès qualitatif*, en termes de confort, de plaisir, voire de profit intellectuel.

Une telle mesure peut éventuellement contribuer à accroître la population de base en incitant à venir des usagers potentiels qui se tenaient jusqu'alors écartés de la bibliothèque, mais elle jouera aussi sur la structure de la population qu'on trouve dans les salles par le jeu des changements de modes d'usage qu'elle aura induits. On pourra ainsi trouver une plus forte proportion de pères de famille engagés dans la vie active, même si ceux-ci réussissaient à s'arranger auparavant pour " passer en vitesse » de temps à autre. Venant plus souvent, restant plus longtemps, ils modifient la composition de la population des usagers présents dans la bibliothèque.

\section{LES ENSEIGNEMENTS D'UNE ÉTUDE FRANCILIENNE : 6 CATÉGORIES DE PUBLIC}

Au cours de l'année 2011, « Plein Sens » en collaboration avec le MOTif a conduit une étude auprès d'un panel contrasté de 5 bibliothèques franciliennes sur l'influence des horaires sur les usages des publics ${ }^{2}$. Certains de ces établissements étaient ouverts le dimanche et parfois en nocturne durant la semaine.

L'étude portait sur les réactions des usagers par rapport aux horaires, sans toutefois être focalisée a priori sur la composition des publics. Mais très vite, il est apparu qu'il était essentiel de distinguer entre divers profils d'usagers pour présenter des résultats qui soient porteurs de sens.

2. Plein Sens/MOTif, Happy Hours. [En ligne] : < www.lemotif.fr >, rubrique Études et analyses, 2011. 
La typologie qui va être développée ici est sans doute largement applicable à un grand nombre d'établissements. Les bibliothèques retenues dans l'étude étaient très différentes les unes des autres, tant dans leur offre que dans leur environnement sociologique.

À partir de cette catégorisation, on peut toutefois concevoir qu'ici ou là une nouvelle catégorie non mentionnée ici apparaisse ou qu'une catégorie mérite d'être scindée en deux catégories nouvelles. Il paraît cependant intéressant de décrire les phénomènes observés à partir de ces 5 établissements franciliens de façon à alimenter la réflexion sur le public d'une bibliothèque.

Les types de publics se distinguent par leur nature et par leur mode d'usage de la bibliothèque. Voici les 6 profils qui sont ressortis de l'étude et leurs caractéristiques en matière de comportement :

1.Les étudiants (et lycéens) Ils peuvent être minoritaires si l'on considère la population globale, mais ils peuvent être les plus nombreux parmi la population des usagers présents dans les salles. En effet, ils sont de gros utilisateurs des espaces, à la fois par la fréquence et par la durée. Ils viennent souvent et longtemps, mais en revanche font rarement usage des collections. Ils ont une forte " disposition à » venir dans des horaires encore plus élargis que ceux qui leur sont proposés, le soir ou le week-end.

2. Les adultes accompagnés d'enfants Sans surprise, c'est surtout le mercredi et les week-ends qu'on trouvera dans les espaces les parents avec leurs enfants. Cette catégorie est absente en soirée, consacrée au repas en famille. Les pères sont peu nombreux en semaine, ils sont très présents en revanche le samedi ou le dimanche.

3. Les actifs fortement diplômés Ils sont peu nombreux en semaine, ils viennent davantage le week-end. Ils passent rapidement et surtout empruntent beaucoup. 
4.Les actifs moins diplômés. On les voit davantage en semaine que les plus fortement diplômés. Ils demeurent un peu plus longtemps dans les salles. Ils empruntent beaucoup de DVD.

5. Les retraités. Ce sont les plus libres de leur temps. Ils viennent pour lire la presse, pour se distraire. On les trouvera dans les salles à des horaires variés, mais beaucoup moins en soirée. De tous les usagers, ce sont les plus indifférents aux horaires d'ouverture : avec moins de contraintes, ils peuvent s'adapter sans problème.

6. Les demandeurs d'emploi. Comme les étudiants, ils peuvent venir souvent et longtemps. Contrairement à ces derniers, ils sont le plus souvent seuls. Ils utilisent beaucoup les ordinateurs. Ils sont également très demandeurs d'horaires aussi élargis que possible.

Ces différents types d'usagers ne se rencontrent pas aux mêmes moments dans la bibliothèque, ou, tout au moins, pas dans les mêmes proportions. La population des usagers venant en bibliothèque varie considérablement d'une période à l'autre.

On relatera ici les résultats globaux de l'étude en rappelant que d'une bibliothèque à l'autre, les données peuvent varier, les différences étant dues notamment à la situation de l'établissement, plus ou moins proche d'une forte concentration urbaine, ou de quartiers où les entreprises sont nombreuses. L'enquête a porté sur la population globale des usagers venant en bibliothèque (interrogés en sortie). Si elle avait été réalisée sur la population des usagers présents dans les salles, les résultats seraient différents. 


\section{CATÉGORIES DE POPULATION/HORAIRES} ENCADRÉ DE FRÉQUENTATION - ENQUÊTE 2011

En semaine, ce sont les retraités qui représentent la catégorie la plus nombreuse. Ils sont suivis de près par les usagers engagés dans une activité professionnelle. Ainsi, contrairement à une idée reçue, les actifs peuvent venir en bibliothèque en semaine. En poussant l'analyse, on s'aperçoit cependant qu'il s'agit d'une catégorie particulière d'actifs, ceux qui sont les moins diplômés. Pour les plus faibles degrés de qualification, les horaires de travail sont souvent décalés ou morcelés, ce qui leur donne du temps libre au moment où la bibliothèque est ouverte, possibilité plus rare pour les cadres et autres professions de grade supérieur.

Les étudiants et demandeurs d'emploi sont également présents. Quoique un peu moins nombreux, ils peuvent constituer la majorité des usagers présents dans les salles parce qu'ils y restent plus longtemps.

Le mercredi est le jour des enfants. Ceci se vérifie naturellement dans les bibliothèques. Deux phénomènes vont de pair avec cet afflux : une nette augmentation du nombre d'emprunts ce jour-là, et une plus grande féminisation du public adulte. Contrairement au public ordinaire des jours de semaine, plus tourné dans l'ensemble vers l'usage de la bibliothèque comme lieu de travail ou de consultation d'Internet ou de la presse, ce sont les collections qui attirent les enfants et leur famille. Ce sont des femmes (mères, grands-mères...) qui accompagnent les enfants, les pères apparaissant plus nombreux pendant les fins de semaine, le samedi et surtout le dimanche.

Lors des ouvertures en soirée, les enfants et les retraités sont peu présents. On constate dans les salles la présence d'un public jeune et studieux, surtout des étudiants. Les actifs forment également une des catégories les plus nombreuses à passer en bibliothèque à ce moment-là.

Le samedi voit une large proportion d'actifs, cadres moyens ou supérieurs, le matin. Ceux-ci passent à l'occasion d'une autre activité, comme des courses dans le quartier. Ils ne restent que peu de temps.

Le samedi après-midi est le moment où la fréquentation est la plus grande. Les profils et les usages y sont très diversifiés. Dans plusieurs des bibliothèques de notre échantillon, on note la présence de beaucoup de jeunes adolescents venus seuls, mais les parents sont également nombreux.

Le dimanche est la journée la plus masculine : $58 \%$ des usagers interrogés sont des hommes (contre $45 \%$ sur l'ensemble des jours d'enquête). C'est le jour où les sorties familiales sont le plus favorisées, notamment celui 
où les pères ont le plus de temps pour s'occuper de leurs enfants et faire avec eux cette sortie en bibliothèque. Les emprunts sont également nombreux. Sans surprise, les usagers du dimanche sont ceux qui sont le plus attachés à l'ouverture de la bibliothèque ce jour-là.

Sur l'ensemble des usagers interrogés au cours de l'enquête, une large majorité se prononce en faveur de l'ouverture de la bibliothèque le dimanche. Il est à noter que des différences sensibles apparaissent d'un site à l'autre. Des diffé- rences apparaissent également entre les catégories d'usagers. Pour les étudiants et les demandeurs d'emploi, la proportion de ceux pour lesquels l'ouverture dominicale " répond à un véritable besoin " rejoint presque celle de ceux qui considèrent simplement que " c'est un avantage appréciable ». En revanche, pour les autres, un peu plus d'un quart considèrent que c'est inutile, beaucoup d'entre eux estimant que « cela va contre le principe du congé du dimanche».

\section{VERS PLUS D'USAGES POUR PLUS D'USAGERS}

Le public de la bibliothèque est de nature à varier en fonction de l'organisation de l'offre. On peut modeler en partie un public par des choix en termes d'accueil, de collections ou d'accès.

Le public d'une bibliothèque est une réalité fluctuante qui peut varier en fonction de l'offre qui lui est proposée. On voit, à partir des observations réalisées dans l'étude réalisée par le MOTif que des publics différents sont attirés par des facilités différentes. Le public se construit en partie par les choix réalisés par la bibliothèque elle-même. Chaque établissement se trouve bien sûr déterminé par des conditions pratiques et par un environnement sociologique spécifique. Mais à l'intérieur de ces cadres, beaucoup d'évolutions sont possibles, avec pour objectif de répondre plus ou moins aux intérêts et modes de vie de tel ou tel public.

Les étudiants seront nombreux là où la bibliothèque multiplie les espaces de travail, avec des horaires étendus (sauf s'il n'y a pas d'établissements d'enseignement dans la région). L'ouverture élargie en week-end va accroître les visites familiales, avec une plus grande consultation des 
ouvrages, et une augmentation des prêts (cela dépend aussi de la localisation de la bibliothèque).

Dans tous les cas, les changements doivent être pensés de manière globale. Une modification des horaires peut s'accompagner de la mise en place de dispositifs spécifiques adaptés, voire d'animations ou d'accueils particuliers.

Il est important de se rappeler que les horaires sont une donnée très mal connue des usagers, y compris de ceux qui viennent régulièrement. Un effort particulier doit être fait à cet égard pour bien communiquer sur cet aspect, en essayant de toucher également les usagers potentiels qui n'imaginent pas que la bibliothèque puisse être ouverte aux moments qui leur seraient utiles. Un avantage destiné à tel ou tel public d'usagers reste lettre morte s'il n'arrive pas à la connaissance de ces derniers.

En définitive, même s'il reste tributaire des réalités de l'environnement, le public de la bibliothèque se construit en fonction des choix qui sont effectués par les responsables. Plus l'offre sera adaptée de manière diversifiée aux différents types de public, plus la fréquentation et l'usage seront développés et la bibliothèque remplira sa mission d'ouverture et de lien culturel, éducatif et social. 


\section{5}

\section{UNE SITUATION PARADOXALE ET CONTRASTÉE}

par

Georges Perrin

\section{UN FAISCEAU DE PARADOXES}

De son rôle traditionnel de gestion et diffusion de documents, la bibliothèque a évolué depuis de nombreuses années vers celui de lieu d'accueil et d'accès à une grande variété de services proposés désormais au public, et ce, dans le cadre de sa vocation culturelle, éducative et sociale. Les efforts importants déployés par les collectivités territoriales ou les universités pour construire et ouvrir de nouveaux espaces ou pour en améliorer le confort, l'accroissement significatif et la diversification des collections, la parfaite maîtrise du numérique et des possibilités qu'il offre à la diffusion documentaire dans tous les domaines, le travail en relation avec des partenaires de plus en plus nombreux du cadre associatif ou institutionnel, tout cela a concouru à transformer radicalement l'image de la bibliothèque. On en veut pour preuve l'abondante littérature qui émane des observations et études diverses sur la nouvelle appréhension de la bibliothèque par les usagers et les comportements renouvelés qu'on y observe.

Et pourtant! Malgré ces constats établis depuis plus de cinq ans, il semble que les bibliothèques ne parviennent pas à accueillir de nouveaux usagers, et notamment ceux qu'elles souhaiteraient conquérir, dans des secteurs de la population encore éloignés d'elles. Il peut, en effet, paraître étrange que des lieux devenus aussi attractifs ne drainent pas davantage d'usagers potentiels. Mais, à y regarder de plus près, il semble que cette situation, pour le moins paradoxale, ne soit pas appelée à perdurer indéfiniment. Il se peut qu'une étude plus fine de ces apparents paradoxes permette de trouver une issue positive, que certaines bibliothèques ont déjà pu emprunter. 


\section{DES LOCAUX POUR AMÉLIORER LA QUALITÉ DE L'ACCUEIL}

Le premier paradoxe concerne l'amplitude relativement faible des horaires d'ouverture rapportée aux efforts d'investissements consentis par les collectivités locales et les universités au cours de ces 25 dernières années. Le constant développement de la lecture publique, qui s'est traduit par la création de nombreuses bibliothèques, l'augmentation importante du nombre de personnels formés, de remise à niveau des budgets de fonctionnement et d'acquisition, n'a pas permis à la moyenne des horaires d'ouverture d'augmenter de façon significative. Comment comprendre que les millions de $\mathrm{m}^{2}$ nouveaux ouverts au cours de cette période ne puissent pas davantage être investis par un public en attente de services dans le domaine de la lecture, de la culture et de l'information?

Dans les témoignages et les projets en cours exposés dans ce volume, on voit bien que, pour les promoteurs de ces ouvertures élargies, la réflexion a consisté dans un premier temps à étudier le parti qui pourrait être tiré de la qualité et du volume de ces nouveaux espaces. En effet, ils n'ont pas considéré les locaux de leur bibliothèque comme de simples surfaces neutres et purement fonctionnelles, mais comme un atout essentiel pour l'amélioration de la qualité de l'accueil du public. Pour les uns, il s'agit d'accueillir, dans le cadre d'un partenariat précis, d'autres services que ceux de la bibliothèque. Pour les autres il convient d'utiliser avec souplesse ces espaces en pratiquant des horaires d'accueil de façon permanente ou ponctuelle, notamment aux moments où les usagers en ont le plus besoin.

\section{une modernisation des outils de travail moins simplificatrice que coûteuse en personnel}

Autre paradoxe : au cours des 20 dernières années, la modernisation des outils de travail s'est considérablement accélérée. L'informatisation des catalogues et la création de catalogues collectifs entraînant la mutualisation du travail bibliographique, l'automatisation des prêts-retours, la numérisation des fonds spécialisés et leur accès en ligne sur place et à distance, ont constitué autant d'avancées susceptibles d'alléger de façon significative le travail interne. On constate au contraire qu'au lieu de libérer du temps de travail pour le personnel, ces procédures de simplification 
ont entraîné la spécialisation de postes de travail qui ont pris de plus en plus d'importance à la fois temporelle et organisationnelle. On voit également, à travers certains exemples développés au long de ces pages, que certains ont souhaité privilégier la polyvalence raisonnée des compétences des personnels, ce qui a permis une répartition plus équilibrée du temps de travail entre la nécessaire gestion des collections et une médiation de qualité auprès du public.

\section{services à distance et accueil de proximité plus complémentaires qu'opposés}

Dernier paradoxe : de nombreuses bibliothèques développent et améliorent sans cesse les services à distance et/ou hors les murs, et c'est, a priori, une excellente orientation pour prendre en compte les nouvelles attentes des usagers. Mais alors se pose la question de l'accueil de proximité au regard de ce développement des services à distance. Ces deux types d'accueil, l'un virtuel, l'autre matériel et très concret, sont-ils suffisamment complémentaires ? Ici encore la démonstration semble faite que les locaux plus largement ouverts permettent un meilleur accueil de l'ensemble des usagers, et a fortiori de ceux qui découvrent la bibliothèque par sa communication à distance.

Toutefois, un certain nombre de bibliothèques, même si elles sont encore trop peu nombreuses, ont fait l'effort d'étendre leurs horaires d'ouverture, soit en repoussant à une heure plus tardive leur fermeture en semaine, soit en pratiquant une ouverture le dimanche. De ce fait, la carte des horaires est très diversifiée, selon les catégories de bibliothèques ou selon les régions. C'est ce que nous allons voir maintenant plus en détail.

\section{DES BIBLIOTHĖQUES MUNICIPALES PEU ENCLINES À OUVRIR DAVANTAGE}

En 2009, le ministère de la Culture et de la Communication, dans le cadre des 14 mesures pour le livre et la lecture, a créé un dispositif d'incitation à l'extension des horaires d'ouverture, accordant aux municipalités qui le souhaitaient, une aide financière en soutien aux initiatives de recrutement 
de vacataires et autres dispositifs permettant un élargissement significatif des ouvertures, notamment l'ouverture du dimanche. Cinq ans après, il semble que cette proposition n'ait recueilli qu'un faible écho.

Depuis quelques années grâce à de nombreux rapports et documents, les principaux défauts et blocages du système actuel sont désormais largement identifiés.

Tout d'abord, dans les bibliothèques municipales, quelle que soit leur dimension, l'amplitude des ouvertures reste médiocre.

\section{L'AMPLITUDE HORAIRE DES BM}

\section{ENCADRÉ EN FRANCE : QUELQUES CHIFFRES}

De 20 heures d'ouverture hebdomadaire (moyenne nationale englobant les plus petites bibliothèques) aux 40 heures d'accueil par semaine dans les plus grandes villes, l'amplitude horaire des ouvertures de nos bibliothèques est assez inférieure à celle de la plupart des bibliothèques de nos voisins européens.

À regarder dans le détail la répartition de ces horaires, on aperçoit le nombre très relatif de bibliothèques qui pratiquent l'ouverture du dimanche (moins de $20 \%$ ). On constate également le nombre restreint de bibliothèques ouvertes le lundi dans les villes de plus de 20000 habitants (17,4 \%). Sur le même échantillon, les ouvertures jusqu'à $20 \mathrm{~h}$ ou au-delà sont encore plus rares $(13,4 \%)$.

À cette étroitesse des plages d'accueil s'ajoute souvent la discontinuité de celles-ci : alternance de demi-journées et de journées complètes, fermetures en milieu de semaine, coupure méridienne, horaires différents selon les espaces, etc. Dans de trop nombreux cas, le tableau des horaires d'ouverture est peu lisible et, de ce fait, n'encourage pas la venue du public.

Certes, les obstacles à un élargissement sont nombreux, et nous verrons plus loin comment tenter de les franchir avec succès. On parle souvent de la médiocre qualité des lieux, de la situation de la bibliothèque au sein de la ville, peu propice à une fréquentation aisée, de la configuration d'espaces cloisonnés et dispersés, qui nécessitent parfois la présence de nombreux personnels pour être accessibles. Et, en effet, avant de mettre en œuvre une ouverture le dimanche ou en nocturne, il s'agit de conduire 
une étude sérieuse afin, peut-être, d'envisager d'autres solutions moins coûteuses en personnel.

Mais on parle plus souvent et de façon plus générale, de l'insuffisance des moyens humains ou financiers pour ouvrir davantage. Cet argument mérite toutefois qu'on l'examine avec une attention plus fine. Certes des renforts sous forme de vacations seront nécessaires pour permettre d'assouplir les plannings, mais, nouveau paradoxe : on constate d'une part que les bibliothèques qui font l'effort d'ouvrir plus ne sont pas celles qui sont les mieux pourvues en personnels ${ }^{1}$. Ceci encourage à réfléchir positivement à une nouvelle organisation du travail et de nouvelles répartitions des tâches dans les bibliothèques qui ont actuellement le plus de difficultés à envisager de nouveaux horaires d'ouverture.

\section{DES BIBLIOTHĖQUES UNIVERSITAIRES EN PROGRÈS}

Grâce à une attention soutenue et une incitation financière du ministère de l'Enseignement supérieur et de la Recherche, la politique d'extension des ouvertures a connu plus de succès au sein des universités que du côté des villes. En effet, d'une part, la mise en œuvre de la loi relative aux libertés et responsabilités des universités (LRU), source du renouveau pour la gouvernance des universités, et le souci de plus en plus prégnant d'une insertion réussie des étudiants dans l'université et ultérieurement dans le monde du travail, ont permis de mettre en évidence les possibilités offertes par les BU : leur capacité d'accueil et leur rôle incontournable dans l'intégration des étudiants au sein de l'université. Dans certaines régions, les collectivités territoriales ont pu apporter un concours financier pour contribuer à l'augmentation des budgets nécessaires au recrutement d'étudiants venant en appui aux personnels titulaires pour accroître les horaires d'ouverture. C'est notamment le cas de la région Île-de-France qui a développé un système de subventionnement pour ce type de recrutements.

1. Voir Georges Perrin, Améliorer l'accueil en bibliothèque : propositions pour une extension des horaires d'ouverture, Rapport $\mathrm{n}^{\circ}$ 2008-001 de l'Inspection générale des bibliothèques, avril 2008, pp. 12-13. 
Ainsi, des efforts ont pu être initiés au cours des deux dernières décennies, permettant, d'approcher la moyenne de 60 heures d'ouverture hebdomadaire des bibliothèques universitaires.

Encore faut-il, en entrant dans le détail, faire la part des secteurs les plus avancés dans ce domaine, et de ceux qui sont encore appelés à faire des efforts pour atteindre un niveau correct d'ouverture. Il est clair que les avancées les plus significatives ont eu lieu dans les sections de santé, très demandeuses en matière d'accueil tant en journée qu'en soirée, tant en semaine qu'au long des week-ends. À cet égard, l'exemple de la Bibliothèque de Santé de l'université de Nantes est intéressant, qui réunit tous les ingrédients de la réussite : la volonté déterminée des autorités universitaires de prendre en compte les demandes des étudiants, la situation géographique de la bibliothèque au cœur de la ville, l'attractivité de ses espaces, la modulation des services offerts après 19 h, l'avancée progressive après évaluation régulière des pratiques et demandes du public, etc. Il est, certes, relativement aisé de développer des horaires d'ouverture au sein d'une institution qui reçoit un public plus spécialisé et plus " captif » que celui des bibliothèques de lecture publique, et dont l'exigence porte, pour une très grande part, sur un nombre de places suffisant pour travailler dans le calme et non prioritairement sur une très grande variété de services et d'accès aux collections. Toutefois, la nécessité d'introduire de la souplesse dans les plannings, et donc de prévoir le recrutement d'un personnel supplétif est identique en BU et en BM.

Ainsi, grâce aux aides financières précitées, les horaires d'ouverture ont pu connaître un saut quantitatif relativement important au cours de ces cinq dernières années. Cependant, beaucoup reste à faire pour harmoniser les plages d'ouverture sur les réseaux des bibliothèques universitaires. 


\section{L'AMPLITUDE HORAIRE DES BU}

\section{ENCADRÉ EN FRANCE : QUELQUES CHIFFRES}

Suivant les disciplines auxquelles elles sont rattachées, les BU n'ont pas la même amplitude d'ouverture.

Les bibliothèques de lettres et sciences humaines ouvrent en moyenne 58 heures par semaine, celles de sciences $58 \mathrm{~h} 30$, et celles de droit 59 h 30. Elles sont encore loin des 65 heures d'ouverture des bibliothèques de santé.

De plus, près de $30 \%$ des BU ne sont pas ouvertes le samedi, jour où les étudiants sont le plus libres pour effectuer du travail personnel ; $50 \%$ sont fermées dès 19 h, et seules $19 \%$ ouvrent au-delà de $20 \mathrm{~h}$.

Quant à l'ouverture du week-end, notamment celle du dimanche telle que la pratique déjà la BU santé de Nantes, elle pourrait s'envisager en concertation avec les bibliothèques municipales qui sont également ouvertes ce jour-là, afin de décharger ces dernières de la pression des étudiants qui l'investissent le dimanche à défaut d'un autre lieu plus adapté à leur attente.

Une démarche identique pourrait réunir les bibliothèques universitaires parisiennes, la Bpi ainsi que la BnF, afin d'offrir aux étudiants parisiens des espaces d'accueil le dimanche, et soulager ainsi ces 2 grandes bibliothèques, même si leur accès n'est interdit à aucune catégorie de population. Il convient de souligner que, contrairement aux universités françaises, de nombreuses universités étrangères gardent leurs bibliothèques ouvertes durant tout l'été. Certaines insistent même sur le fait que le mois d'août connaît une des fréquentations les plus importantes de l'année. C'est en effet la période où les étudiants étrangers en résidence à l'université ont besoin de la bibliothèque, et celle où les chercheurs des autres universités viennent consulter les documents nécessaires à la poursuite de leurs travaux.

Nous avons déjà évoqué, à propos des bibliothèques municipales, quelques obstacles à l'extension des horaires d'accueil du public. Les bibliothèques universitaires, elles, sont tributaires des rythmes " scolaires » de leurs universités qui, la plupart du temps pour des raisons d'économie, ne souhaitent pas garder de services ouverts durant les vacances de Noël ou 
d'été. Mais, dans la mesure où la bibliothèque est reconnue comme un des lieux de vie essentiels des étudiants, ne serait-il pas envisageable de ne pas les priver, durant ces périodes, de cet accès à une place de travail et aux documents utiles à leurs études, qui constituent de sérieux atouts pour leur réussite future?

\section{Des perspectives encourageantes : quelques pistes}

Les perspectives d'avancées concernant l'élargissement des horaires d'ouverture semblent reposer sur des prospectives plus affirmées du côté des bibliothèques universitaires que des bibliothèques municipales.

En effet, séduites par le succès avéré d'expériences étrangères, notamment des Learning Centres, plusieurs universités envisagent de tenter l'expérience d'adapter l'organisation d'au moins une section de leur réseau documentaire aux principes de fonctionnement de ces lieux d'un type nouveau : décloisonnement des espaces, diversification des services, qualité du confort et extension des plages horaires d'accueil. Sans doute est-il plus facile pour les équipes de ces bibliothèques que pour celles des bibliothèques municipales de cerner les réelles attentes et les nouveaux comportements de leur public, plus homogène que celui de la lecture publique ?

Quant aux bibliothèques municipales, le succès de celles qui, d'ores et déjà, ouvrent leurs portes le dimanche est suffisamment probant par la qualité des résultats constatés pour constituer autant d'incitations à vouloir enfin concrétiser le souhait si souvent exprimé par les bibliothécaires : accueillir de nouveaux publics dans une ambiance plus familiale, qui répond parfaitement aux attentes d'usagers disposés à utiliser les services qui leur sont offerts dans les meilleures conditions d'accueil et de confort, et selon le meilleur rythme qui soit, celui de la détente et d'une convivialité heureuse. 


\section{6}

\section{OUVRIR LE DIMANCHE? MÉTHODOLOGIE POUR DES NÉGOCIATIONS RÉUSSIES}

par

Jean-François

Jacques

\section{LA DÉCISION POLITIQUE, LES PARTENAIRES}

L'ouverture dominicale des médiathèques est un choix politique : les horaires sont liés à la politique de service public, c'est-à-dire à une analyse des besoins et des attentes des populations, à une définition volontariste d'offre de services nouveaux.

Mais ce choix n'est réalisable qu'à condition que les moyens soient cohérents avec les ambitions, et qu'il ne se fasse pas au détriment des agents (nombre d'agents, rémunérations, conditions de travail...).

L'ouverture dominicale d'une médiathèque est un challenge difficile et passionnant... mais la réussite du projet tient à la rigueur de la discussion préalable avec l'ensemble des parties concernées : élus, agents, syndicats. Ce progrès considérable du service public ne peut se faire au rabais, il faut donc en traiter soigneusement tous les aspects.

Les informations et les conseils qui suivent découlent pour une très grande part de l'observation et de l'évaluation des expériences d'extension des horaires d'ouverture déjà mises en œuvre et qui ont connu le succès. Ils sont donc formulés à partir de la réalité du fonctionnement habituel des bibliothèques aujourd'hui et en tenant compte de l'organisation de la répartition entre travail interne et accueil du public tel qu'il a cours dans la plupart des bibliothèques. Mais certaines bibliothèques travaillent aujourd'hui à une redéfinition de cet « équilibre » en souhaitant accorder davantage de temps au contact avec le public. Cette nuance peut sans doute, à l'avenir, conduire à un réexamen d'une nouvelle répartition des professionnels à l'intérieur de la bibliothèque entre l'accueil du public et la gestion interne du fonctionnement de la bibliothèque. Dans tous les cas de figure, et en raison de la nécessaire souplesse à introduire dans la gestion des plannings, des moyens d'appoint en personnel seront 
nécessaires. Mais dans le cas d'une réorganisation allant dans le sens d'un accueil « augmenté » du public, ce besoin d'appoint pourrait s'avérer moins important ${ }^{1}$.

La décision politique doit être soigneusement préparée : si elle doit être ferme et claire, elle ne doit pas être un " diktat ». Les élus et l'équipe de direction de la collectivité doivent comprendre que la réussite du projet va dépendre de la négociation avec le personnel de la bibliothèque et avec les syndicats, menée avec et par son (sa) responsable, et par la hiérarchie de la collectivité.

Le responsable (ou la responsable : pour ne pas alourdir le texte, je ne répéterai pas cette précision !) de la bibliothèque doit donc être impliqué dans le processus de décision politique, et doit pouvoir présenter aux élus et à l'administration de la collectivité son analyse personnelle de la faisabilité du projet, et des conditions de sa réussite, en amont des autres négociations.

Dans la phase de préparation, le responsable se trouve au cœur d'un débat triangulaire entre les élus, l'administration de la collectivité et son équipe : c'est une position difficile, qui lui demande de faire preuve de pédagogie, de rationalité et de loyauté. Il va de soi que son adhésion au principe de l'ouverture dominicale doit être entière et manifeste : elle fonde la crédibilité de ses propositions aux élus ; mais de sa capacité à écouter pédagogiquement le personnel, à faire remonter rationnellement ses questions et ses demandes dépend, pour une grande part, l'issue du projet.

En dehors de cette relation triangulaire, les syndicats interviennent, parfois en divergence avec l'attitude du personnel. En effet, leurs arguments et leurs motivations concernant les fonctionnaires de manière large, ils peuvent manifester une opposition plus ou moins systématique à des dispositions qui, si elles étaient généralisées sans contreparties, deviendraient des obligations de travail dominical dommageables au personnel. Leur opposition de principe au travail dominical est souvent générale, mais n'exclut pas les accords locaux qu'ils peuvent accepter si les personnels y trouvent leur intérêt.

1. Voir Jean-François Jacques, "Les horaires d'ouverture des bibliothèques : un service public de la culture », Bibliothèque(s), n53/54, 2010, p. 33. [En ligne] : < http://www.enssib.fr/bibliotheque-numerique/documents/59925-53-54-service-public.pdf\#page=35 > . 


\section{MÉTHODOLOGIE DE LA NÉGOCIATION}

Rien n'interdit le travail dominical dans le statut de la fonction publique : la seule obligation est celle du repos hebdomadaire de 35 heures ${ }^{2}$. Beaucoup d'agents travaillent déjà le dimanche (sports, sécurité, etc.) sans compensation. Dans ce contexte, la négociation préalable avec les élus porte sur le principe, l'assurance de moyens nouveaux, et la compensation de la sujétion spéciale : il est impossible de réaliser une telle évolution du service aux publics par une simple réorganisation, que les élus envisagent légitimement. Le responsable de la bibliothèque doit prouver que son organisation est déjà optimale, et entièrement orientée « services aux publics » : une très large moitié du temps de travail est consacrée à l'accueil, aucun catalogage ni révision de catalogue ne sont faits sur place, la chaîne d'acquisition et de traitement des livres est optimisée. Il doit sortir de cette première phase avec l'assurance de moyens et de compensations qui lui permettront la négociation avec son équipe.

Avec les agents, le responsable avance l'argument du développement indispensable des services aux publics, pour une meilleure réalisation des missions de la bibliothèque. Il souligne que la bibliothèque est le seul équipement culturel à ne pas être accessible le dimanche, et que ce jour de très nombreuses personnes sont au service des populations : santé, transports, sécurité, alimentation, etc. Il s'appuie sur l'assurance donnée par les élus qu'il y aura moyens nouveaux et compensations. Aucune demande ne doit être écartée : dans une négociation, aucun argument ne peut être méprisé. Le responsable doit hiérarchiser les questions, et proposer de les traiter méthodiquement.

Le développement qui suit a pour objet de lister les questions posées par les uns et les autres. Le responsable de la bibliothèque doit savoir que certaines sont solubles, d'autres pas, mais qu'il importe que toutes soient entendues par lui-même et par les décideurs, élus et administratifs. Celles qui ne peuvent trouver de réponse constitueront vite des obstacles majeurs si elles sont méprisées, ou si les compensations prévues sont insuffisantes.

2. Voir < http://vosdroits.service-public.fr/particuliers/F571.xhtml >. 


\section{CALENDRIER, HORAIRES}

Le calendrier d'application de l'ouverture dominicale est déterminant, tant pour le public que pour le personnel.

La période optimale va du $1^{\mathrm{er}}$ septembre au 30 juin ; arguments en faveur de ces dates : déconnexion du calendrier scolaire, mémorisation facile et répétée d'année en année, meilleure réponse à l'ensemble des besoins, difficulté évitée d'organisation d'une ouverture dominicale estivale.

Des périodes plus restreintes sont choisies quand les moyens sont insuffisants (par exemple, octobre à mai...).

La proposition d'horaires est plus complexe.

L'amplitude optimale est une ouverture toute la journée, identique au samedi, par exemple $10 \mathrm{~h} / 17 \mathrm{~h}$. Elle offre la meilleure réponse aux besoins estimés : on constate que les publics du matin sont différents des publics de l'après-midi. De plus, cette durée étant celle d'une journée de travail normal, l'insertion dans l'organisation des rythmes de travail est meilleure. L'amplitude minimale est la demi-journée. Le matin (exemple $9 \mathrm{~h} / 12 \mathrm{~h}$ ), la durée est courte mais peut être efficace suivant les circonstances ; les agents restent libres l'après-midi ; mais la réponse aux besoins est moyenne. L'après-midi (exemple $14 \mathrm{~h} / 18 \mathrm{~h}$ ) la durée étant plus longue, la réponse à un grand nombre de besoins est assurée, il n'y a pas de problème de repas à prendre sur place ; mais les agents ne seront rémunérés que pour une demi-journée, alors que leur journée entière est pratiquement bloquée.

\section{SERVICES OFFERTS : ALTERNATIVES}

Le service peut être limité au prêt-retour/renseignements : moins d'agents nécessaires, mais le public est potentiellement frustré, et « l'appel » auprès des nouveaux publics attirés par les services est moindre.

Avec une ouverture de tous les services (réservations, informatique avec assistance, ateliers, etc.), les publics spécifiques du dimanche sont mieux desservis et plus satisfaits. Mais l'exigence en personnel est maximale, l'expérience montrant que le dimanche est souvent le plus " gros » jour de la semaine.

L'offre d'animation est une charge supplémentaire potentiellement importante, mais l'expérience montre un grand succès : projections, mini-concerts, 
conférences, heures du conte, etc. Les élus la demandent souvent, pour la « visibilité » qu'elle donne à l'extension des horaires.

\section{CONDITIONS ET RYTHME DE TRAVAIL}

Pourquoi examiner ces points et la question de la rémunération avant de parler de l'organisation?

Parce que l'expérience montre que leur prise en compte correcte est la clé de voûte de la faisabilité du projet, conditionnant la motivation des agents et la sérénité du débat.

Les aspects suivants doivent être examinés et correctement résolus. Mais il est clair que certains d'entre eux ne peuvent matériellement pas trouver de solution, et vont donc accroître les difficultés des agents, et les contraintes du travail dominical (le terme de contrainte est plus adéquat que «pénibilité »), justifiant les compensations :

- transports : les moyens de transport collectifs habituellement utilisés par les agents sont-ils disponibles le dimanche?

- certains agents ont-ils des difficultés temporaires ou permanentes de garde d'enfants?

- quelles seront les conditions de prise du repas de midi ?

- le rythme de travail des agents ne devrait pas être supérieur à un dimanche sur quatre : la fixation de ce rythme conditionne toute l'organisation, et détermine le nombre de postes nécessaires de titulaires et de non-titulaires ;

- faut-il une obligation pour tous les agents ? faire appel au volontariat? faire appel au réseau quand il existe?

- l'appel au volontariat pur s'avère difficile : il peut ne pas être possible de réunir le nombre d'agents nécessaire. À terme, ce choix peut aussi être perçu comme inégalitaire ;

- il est sans doute préférable de faire le choix inverse : faire examiner par une commission ad hoc la demande d'agents qui auraient des raisons précises et très légitimes de ne pas travailler ce jour-là ; 
- l'obligation est plus facile à instaurer à l'ouverture d'un équipement, ou lors d'une importante transformation.

\section{PERSONNELS}

\section{AGENTS TITULAIRES}

La question de l'encadrement doit être examinée dès le départ, en particulier dans les petits et moyens établissements. Quel nombre de cadres A ou $\mathrm{B}$ ou $\mathrm{B}+$ assumeront la responsabilité de la bibliothèque le dimanche ?

Le nombre d'agents présents est un élément délicat. Il ne doit pas être sous-estimé : quelle que soit l'amplitude d'ouverture, c'est le plus gros jour de la semaine ; il ne doit pas y avoir de "travail interne ", il faut donc ménager des temps de pause de 15 à 20 minutes entre les plages de services publics de 1,5 à 2 heures; il ne faut pas établir de rotation de toute l'équipe d'un seul coup, et dans le cas d'une ouverture toute la journée, prévoir le temps du repas.

Habituellement, le nombre optimal de postes dans une médiathèque est de 2,5 à 3 fois le nombre d'agents nécessaires en service en présence du public. Si l'on veut que les agents ne travaillent qu'un dimanche sur 4, cela veut dire qu'on ne pourra compter que sur un cinquième au mieux des agents (congés, absences diverses). Par exemple, si l'on a besoin de 10 agents pour ouvrir en moyenne en semaine, 12 les jours de grande affluence, l'équipe doit compter 25 postes : on mobilise chaque dimanche 5 titulaires, et donc il faudra 5 agents non-titulaires au moins, 7 pour bien faire. On a dans cette question la clé de la réussite du projet : les élus doivent absolument en avoir une idée très claire, et ne pas voir dans ce raisonnement une tentative de maximiser le nombre de postes nécessaires. Les échecs ou les conflits liés à l'ouverture dominicale ont le plus souvent leur source dans cette question.

L'équipe titulaire doit être aidée par un nombre suffisant d'agents nontitulaires : la parité (souvent réclamée par les syndicats, comme un préalable) ne doit pas constituer une limite, d'autant que le choix du recrutement d'étudiants (voir infra) permet d'avoir des personnes rapidement compétentes. 


\section{AGENTS NON-TITULAIRES À TEMPS NON COMPLET (ANTTNC)}

L'argument de la lutte contre les emplois précaires est recevable. Il est préconisé de limiter exclusivement et contractuellement ces recrutements à des étudiants : ils sont demandeurs de ce type d'emploi, plus « motivant " que la restauration rapide ; ils sont rapidement et efficacement formés ; ce type d'emploi est bien connu en BU ; des aides à l'emploi étudiant en bibliothèque peuvent être apportées par les régions ; dans certains cas, ils peuvent être essentiellement habitants de la collectivité. L'obstacle principal est que la fonction publique ne prévoit de contrats à durée déterminée (CDD) que pour des périodes de 6 mois au maximum, définis " emplois saisonniers " : il est souhaitable que cette autorisation soit portée à 9 ou 12 mois dans les conditions exclusives des emplois étudiants, par application des dispositions prises dans la fonction publique d'état (voir le décret n 2007-1915 du 26 décembre 2007 publié en annexe du rapport Perrin) $)^{3}$.

Il faut noter que certaines communes recrutent des titulaires du RSA, des demandeurs d'emploi.

\section{RÉMUNÉRATION}

Les indications données ici, à la date de rédaction de cette contribution, ne peuvent tenir compte des aides de l'État annoncées sans précision en mars 2014.

Le travail dominical, même occasionnel, est une contrainte lourde, qui nécessite une compensation importante : un doublement de la rémunération ou une récupération + compensation double ne sont pas illégitimes ; certains textes le prévoient ou aboutissent à ce résultat, mais pas pour tous. Je pense que la création au niveau national d'une rémunération statutaire spécifique est un vrai challenge, qui conditionnerait favorablement toute évolution de cette offre de service.

3. Georges Perrin, Améliorer l'accueil dans les bibliothèques : propositions pour une extension des horaires d'ouverture, op. cit. 


\section{ENCADRÉ RÉMUNÉRATION DU TRAVAIL DOMINICAL}

** Si l'accord local sur les 35 heures ne prévoit pas la possibilité d'heures supplémentaires, le temps de travail dominical est nécessairement inclus dans le rythme de travail. On organise en alternance des semaines « longues » alternant avec des semaines « courtes » incluant la ou les journées non travaillées entraînées par un dimanche travaillé (il ne s'agit pas là de « récupération » au sens propre, mais de planning).

Il n'est pas nécessaire de figer le règlement (ex. " tous ceux qui ont travaillé un dimanche ne travaillent pas le samedi qui suit ») : l'expérience montre que l'organisation du service gagne à de la souplesse.

La compensation de la contrainte $\mathrm{du}$ travail dominical peut se faire en temps de repos supplémentaire (ex. : pour un dimanche travaillé, deux jours non travaillés en semaine, consécutifs ou pas: pas de réglementation, pas de délibération, une simple note du directeur général des services suffit dans ce cas). Les contraintes sur le service sont lourdes.
La compensation peut consister en une rémunération supplémentaire spécifique, souvent appelée " prime ». La prime peut être prise sur une des « enveloppes » prévues pour les collectivités territoriales (IFTS, IAT) ou faire l'objet d'une décision spécifique, ce qui semble avoir été toléré. Rappelons que le taux d'IAT peut aller de 1 à 8 .

** Si les accords locaux autorisent les heures supplémentaires, on se réfère aux textes en vigueur au moment de la négociation. Des décrets prévoient pour l'ensemble des catégories B et C un taux d'IHTS majoré pour les premières heures, suivi d'une majoration pour le travail dominical. Ces IHTS sont cumulables avec l'IAT et les IFTS, mais pas avec un repos compensateur ni avec l'indemnité précédente.

** Pour les agents de catégorie A, rien n'est prévu spécifiquement : il faut alors négocier dans le cadre des indemnités existantes ou tenter un régime indemnitaire spécifique. La création d'indemnités spécifiques n'est pas impossible ; la situation peut donc différer suivant les collectivités...

\section{ORGANISATION}

En fonction du nombre d'agents titulaires et non-titulaires, une gestion des dimanches doit être organisée ; cette gestion peut prendre une demijournée par semaine. 
Un système doit être mis au point et codifié, approuvé par les agents et la collectivité.

Il prévoit la gestion du remplacement le dimanche en cas de congé d'un agent ; les échanges pour convenances personnelles, indispensables ; l'accueil téléphonique ; la régie de recettes quand elle est indispensable, etc. L'organisation des plannings doit être adaptée : plages plus courtes qu'en semaine ; " profils de poste " précisément déterminés ; présence des responsables et rôle soigneusement définis.

\section{Les agents non-titulaires à temps non complet : rémunération, recrutement, formation et gestion}

Les textes permettent des contrats de travail souples : un nombre d'heures mensuelles fixé, les jours et horaires de travail est établi par les plannings avec 3 ou 4 semaines d'avance.

$\rightarrow$ La rémunération. Elle est très variable selon les collectivités, variant, dans une proportion de 1 à 2 à partir du taux minimal (SMIC). S’y ajoutent congés et prime de précarité. Elle est mensualisée : seuls les "vacataires » des collectivités sont payés à l'heure, mais ce statut ne convient pas aux bibliothèques, recouvrant des tâches ponctuelles, brèves, non répétitives. Par contre, la rémunération de base s'accompagne d'une compensation de la contrainte pour travail dominical identique à celle des titulaires. Une rémunération correcte est un gage de fidélisation et de stabilité : attention aux défections en période d'examens - en mai et juin ! Elle conditionne l'exigence dans la qualité du service et de l'accueil. La rémunération doit fidéliser !

$\rightarrow$ Les ANTTNC travaillent un week-end sur deux - il est préférable qu'ils travaillent le samedi et le dimanche. En fonction du régime adopté pour les titulaires, cela peut être nécessaire. Ils peuvent aussi être disponibles en semaine pour des coups de main... mais cela dépend du « capital heures » accordé par la collectivité à la bibliothèque.

$\rightarrow$ Un responsable est indispensable : il organise les plannings, convoque, gère les remplaçants. Il pourra aussi être chargé d'organiser les recrutements. Dans le cas d'une organisation souple, qui prévoit un nombre 
d'heures mensuelles organisées de manière variable en fonction des besoins, des flux saisonniers, cette tâche est complexe mais peut s'avérer très utile pour l'ensemble du service.

$\rightarrow$ Le recrutement des ANTTNC peut débuter par un appel à candidatures en mai, pour un recrutement fin juin/début juillet ; formation fin août/début septembre. De nombreuses communes réservent ces emplois à des étudiants habitant la ville. On peut décider de recruter à bac +2 : il faut éviter que certains étudiants lâchent leurs études du fait de ce travail. On limitera de manière draconienne les contrats à dix mois renouvelables une fois. On peut faire une première sélection sur dossiers, puis procéder à un entretien collectif (une douzaine de candidats au maximum). Il s'agit bien d'un recrutement par la ville : la DRH, les élus, doivent être impliqués comme dans un recrutement traditionnel.

$\rightarrow$ La formation est un atout important pour une efficacité rapide des étudiants recrutés. Elle comprendra :

- un panorama général de l'équipement : missions, place dans la collectivité, notion de "service public », notions sur les méthodes de gestion des collections ;

- un apprentissage du maniement du catalogue et du classement des documents (Dewey...) ;

- un apprentissage des opérations de retours, prêts ;

- ensuite, on place les étudiants en " doublon » pendant une semaine ou deux, avec des titulaires ou avec d'anciens ANTTNC.

\section{GESTION MATÉRIELLE, SÉCURITÉ, COMMUNICATION}

L'affluence nécessite une prise en compte spécifique des questions matérielles.

Il faut procéder à une étude préalable des conditions de sécurité extérieure et de la maintenance technique : le commissariat est-il ouvert ? Qui répondra à un appel d'urgence ? La permanence des services techniques est-elle prévenue, est-elle apte à répondre à un incident ? Y a-t-il une permanence de la gestion informatique ? Qu'arrivera-t-il en cas de 
défaillance du système ? Comment organiser le ménage de la bibliothèque après l'ouverture du samedi ?

Dans les très grands établissements (au-delà de $2000 \mathrm{~m}^{2}$, de 2000 usagers attendus), la solution optimale consiste à prévoir une permanence de ménage pendant l'ouverture dominicale, l'affluence « record » pouvant nécessiter des interventions fréquentes dans les espaces publics, les toilettes... Il ne sert à rien de faire cette offre sans le faire savoir aux publics concernés : une action de communication forte sera donc entreprise, y compris en dehors des moyens traditionnels que sont le journal municipal et l'affichage sur place.

Ces questions sont souvent des obstacles à la réussite du projet. 


\section{PARTIE ITI}

\section{DES PISTES \\ DÉJÀ OUVERTES}

1. 7 jours sur 7 des bibliothèques : du 9-3 à south kensington par Ophélie Ramonatxo

$+++++++++++++++++++++++++++++++++++++++++++++++++++++++++++++++++$

2. Heureux étudiants de Nantes ! Le SCD de l'université de Nantes par Hélène Grognet et Emmanuelle Paulet-Grandguillot

3. " tu travailles ce week-end ? " : les emplois étudiants à la bm de Toulouse par Charlotte Henard

4. ouvrir plus et mieux dans un contexte contraint : à l'INSA de Lyon, l'innovation pédagogique comme moteur d'un accueil élargi par Monique Joly 


\section{1}

\section{JOURS SUR 7 \\ DES BIBLIOTHEQQUES : DU 9-3 À SOUTH KENSINGTON}

par

Ophélie

Ramonatxo

" Je ne peux pas venir vous voir, vous êtes toujours fermés!", me dit un jour un commerçant travaillant à deux pas de la médiathèque de Drancy (93), ouverte $7 \mathrm{j} / 7 . .$. Trois années après l'inauguration de cette dernière, j'atterrissais à Londres, paradis des commerces ouverts non-stop et de l'automatisation des services, enfer du droit du travail et terre promise des bibliothèques ouvertes $7 \mathrm{j} / 7$ et, pour certaines, $24 \mathrm{~h} / 24$. Une performance qui n'a pas empêché les councils britanniques d'annoncer à l'époque la fermeture programmée de près d'un tiers des bibliothèques publiques du pays ${ }^{1} \ldots$

Depuis l'article de Jacques Vidal-Naquet en $1993^{2}$ jusqu'au rapport de Dominique Arot de $2012^{3}$, tout a été dit concernant les horaires d'ouverture des bibliothèques. Bien plus qu'un service comme les autres, ils seraient l'emblème même de la politique de service de la bibliothèque et se devraient, pour cela, d'être partout étendus à leur maximum. Mais ouvrir largement nécessiterait aussi une organisation tout à fait spécifique gestion rationalisée, moyens humains surdéveloppés, conception des bâtiments particulière -, pas forcément accessible à toutes les bibliothèques. Le $7 \mathrm{j} / 7$ des bibliothèques, utopie ou dystopie ? Le militantisme professionnel doit-il s'en faire le défenseur au nom de la démocratie culturelle ou au contraire le détracteur au nom d'une lutte contre la consommation à tout va ? Proposer une bibliothèque très ouverte est quelque chose de relativement " aisé » dans le cas d'une construction d'équipement comme je l'ai expérimenté à Drancy. À l'inverse et dans la majorité des cas, la

1. Ophélie Ramonatxo, "Le "cas" des bibliothèques britanniques », Bulletin des bibliothèques de France, 2011, n² 2, pp. 46-50. [En ligne] : < http://bbf.enssib.fr/consulter/bbf-2011-02-0046-009 >.

2. Jacques Vidal-Naquet, « Les horaires d'ouverture des bibliothèques municipales », Bulletin des bibliothèques de France, 1993, n 6, pp. 8-14. [En ligne] : < http://bbf.enssib.fr/consulter/bbf1993-06-0008-001 >.

3. Dominique Arot, L'extension des horaires d'ouverture des bibliothèques, progrès et obstacles, Rapport n²012-005 de l'Inspection générale des bibliothèques, 2012. 
bibliothèque déjà existante va imposer ses règles et les priorités d'action ne se tourneront malheureusement pas toujours, comme je l'ai expérimenté à Londres, vers l'élargissement des horaires. Les leçons tirées de ces deux expériences en apparence opposées, me permettront néanmoins de suggérer quelques solutions aux problèmes se posant souvent en cas d'ouverture élargie afin de ne jamais perdre de vue cet idéal : ouvrir toujours plus.

\section{QUAND LE TEMPS EST MAÎTRE DU LIEU : L'IDÉAL DRANCÉEN}

On pourrait user de l'illusion rétrospective et dire qu'à Drancy, toutes les conditions étaient réunies pour aboutir à une ouverture $7 \mathrm{j} / 7$ :

- fort volontarisme politique ;

- faible syndicalisme des équipes ;

- architecture et fonctionnement du bâtiment appropriés.

Mais comment a-t-on pu réellement en arriver là, dans une des grandes villes les plus pauvres de France, peu tournée vers la promotion de la culture et dont les structures de lecture publique se positionnaient tout en bas du classement national des bibliothèques?

\section{UNE VOLONTÉ POLITIQUE}

À mon arrivée à Drancy en tant que directrice-adjointe du réseau des bibliothèques municipales (5 établissements), le directeur de l'époque, Alain Pansu, me confia comme première mission la mise au point des horaires d'ouverture de la future médiathèque qui devait être inaugurée 6 mois plus tard. Il me donna une seule directive : " Le maire souhaite qu'on ouvre le dimanche ". Ayant eu écho du succès de l'ouverture du dimanche de la médiathèque d'Issy-les-Moulineaux, ville dont le maire était issu de la même famille politique, Jean-Christophe Lagarde était prêt à fournir les moyens nécessaires à cette mesure inédite en Seine-Saint-Denis. 


\section{UNE NÉGOCIATION FACILE AVEC L'ÉQUIPE}

Forte de cet appui et de ces garanties, je lançais un cycle de réunions de travail auquel je conviais toute l'équipe, même les bibliothécaires des annexes de quartier. Peu syndiqués, les unions de travailleurs étant ressorties très affaiblies de la défaite de la précédente municipalité aux dernières élections, les bibliothécaires n'ont pas émis le désir d'associer les syndicats à ce stade de la discussion. Basée sur des scénarios élaborés et proposés de manière anonyme par les membres même de l'équipe, avec pour seule contrainte celle de respecter la volonté municipale d'ouvrir le dimanche, la négociation a vite abouti à une conclusion : l'équipe était satisfaite des horaires en place et souhaitait y apporter le moins de changements possibles. La bibliothèque centrale ouvrait à cette époque-là du lundi au samedi, grâce à la division de l'équipe en deux groupes, l'un travaillant du lundi au vendredi pendant que l'autre travaillait du mardi au samedi avec une alternance une semaine sur deux qui permettait à chacun d'avoir un week-end de trois jours deux fois par mois.

Les nouveaux horaires d'ouverture n'ont consisté " qu'à » ajouter le dimanche comme une journée supplémentaire, payée ou récupérée en conséquence (payée double pour les catégories $\mathrm{B}$ et $\mathrm{C}$, récupérée double pour les A) et ouvrée grâce à l'appui de neuf vacataires. La présence de quatre titulaires par dimanche était néanmoins nécessaire, mais comme la totalité des équipes du réseau, auquel s'ajouteraient bientôt les bibliothèques du Bourget et de Dugny dans le cadre de la création de la Communauté d'agglomération de l'Aéroport du Bourget, avait accepté de contribuer à l'effort, cela revenait pour chacun à travailler « seulement » 4 dimanches par an tout au plus 4 .

\section{UN BÂTIMENT EN OR}

Mais le secret du 7 j/7 à Drancy, c'est avant tout le nouveau lieu que constituait la médiathèque Georges Brassens. Une architecture toute simple donc extrêmement efficace : grâce à ses trois plateaux de $1000 \mathrm{~m}^{2}$, entièrement décloisonnés et à la disposition du mobilier permettant à un

4. Ophélie Ramonatxo, Drancy 7 jours sur 7, 2011. [En ligne] : < www.bibliotheques93.fr/fichiers/ Drancy77.ppt >. 
seul bibliothécaire d'avoir une bonne visibilité sur l'ensemble de l'étage, la médiathèque Georges Brassens pouvait ouvrir avec un minimum de 5 personnes et un maximum de 9 personnes là où l'équipe comptait 35 agents. À cela s'ajoutait un prêt et un retour centralisés, une gratuité totale qui évitait de passer du temps sur la gestion monétaire et l'introduction de la technologie RFID qui, même sans automate de prêt à l'époque, permettait de réaliser des transactions plus rapides.

Si le projet d'établissement et le cahier des charges de construction prévoient dès le départ de réunir toutes les conditions pour une large amplitude horaire et si l'équipe y est préparée progressivement, sur le long terme, puis associée à l'occasion d'une construction nouvelle, ouvrir largement peut se faire relativement aisément. Si le soutien politique est là, et, par-là, j'entends l'attribution de réels moyens budgétaires, on peut alors passer d'une ouverture large à une ouverture hors du commun.

\section{QUAND LE LIEU EST MAÎTRE DU TEMPS : LE CASSE-TÊTE LONDONIEN}

À mon arrivée à la tête de la médiathèque de l'Institut français de Londres, la situation pouvait sembler propice à un allongement des horaires d'ouverture :

- une bonne base de 34 heures d'ouverture sur 5 jours ;

- un personnel passant seulement $30 \%$ de son temps à l'accueil du public ;

- un institut ouvert $7 \mathrm{j} / 7$.

La situation sur le terrain m'a amenée à un constat tout autre : l'amplitude horaire en apparence satisfaisante était en fait trop importante au vu des moyens alloués à la médiathèque ; les pratiques professionnelles de l'équipe, dépassées, ne permettaient pas de dégager du temps pour que l'équipe fasse davantage d'accueil ; l'environnement institutionnel très demandeur d'espaces événementiels constituait une menace pour la bibliothèque et ses horaires d'ouverture. 


\section{UNE OUVERTURE DÉJÀ TROP IMPORTANTE AU VU DES MOYENS ALLOUÉS}

Avec une ouverture de 12 h à 19 h du mardi au vendredi, à 18 h le samedi, la médiathèque de Londres n'avait pas à rougir de son amplitude horaire. Il est admirable que la réduction constante des moyens dont la médiathèque est victime depuis la fin des années 1990 (division par deux du personnel aujourd'hui de 6,5 équivalents temps plein, [ETP], parallèlement à une multiplication par cinq des espaces aujourd'hui de $500 \mathrm{~m}^{2}$ ) ne se soit pas traduite par une très forte réduction des horaires d'ouverture : à l'exception notable de la bibliothèque enfants dont les horaires sont passés de 34 à 18 heures hebdomadaires suite à la suppression d'un poste en 2006. Néanmoins, en ayant voulu, dans ce contexte, conserver intacts les horaires d'ouverture en bibliothèque adultes sans augmenter la part d'accueil réalisée par chaque agent ni rationaliser les pratiques professionnelles, la bibliothèque a accepté de sacrifier la qualité du service rendu pendant cette ouverture. Si les compétences du personnel en termes d'accueil étaient réelles, la configuration et l'étendue des espaces à surveiller (3 à 4 espaces cloisonnés en bibliothèque adultes ; une bibliothèque enfants dans un bâtiment différent) mais aussi le volume et la diversité des tâches et des collections à gérer (55000 documents sur tous les sujets), permettaient de dire que la médiathèque de Londres vivait réellement au-dessus de ses moyens. Il n'était donc pas question pour moi, dans un premier temps, d'ouvrir plus.

\section{UNE ORGANISATION INTERNE SACRIFIANT LE SERVICE PUBLIC}

Malgré les coupes régulières de budget et de personnel au fil des années, l'équipe de la médiathèque de Londres semblait ne pas avoir changé ses pratiques professionnelles en conséquence et ne s'était pas réorganisée. La politique documentaire visait toujours des collections encyclopédiques et généralistes selon un suivi très scolaire de la Dewey : chacun avait en charge une multitude de petits secteurs d'acquisitions émiettés. Les acquisitions se faisaient pour la grande majorité en France ce qui alourdissait les commandes et les démarches. Pas ou peu d'importations de notices pour ce qui était du catalogage. Des cotes réalisées à la main et un équipement à l'ancienne (film autocollant) comme dans la majorité 
des bibliothèques françaises encore aujourd'hui. Le rangement des collections ne pouvait pas se faire pendant les heures d'ouverture car une seule personne se trouvait à l'accueil en adultes, une seule en jeunesse, et elles étaient occupées par les multiples tâches traditionnelles liées à l'accueil (prêt, retour, inscriptions, information minimale car pas le temps de faire mieux) auxquelles il fallait ajouter quelques spécificités locales : encaissements en espèces des inscriptions, amendes et produits des ventes de livres d'occasion ; remplissage des documents administratifs rendu obligatoires pour de tels encaissements, retrait des boîtiers antivol pour les CD et DVD... Sans parler des multiples tâches internes transversales que la bibliothèque a acceptées de prendre en charge au fil des années parmi lesquelles le Bureau Campus France (service d'information pour les jeunes britanniques souhaitant étudier en France) ou le Service d'information rapide (réponse à toute question d'ordre culturelle posée à l'Ambassade de France, une sorte de « Guichet du Savoir » avant l'heure)...

\section{DES OBLIGATIONS INSTITUTIONNELLES EMPÊCHANT D'OUVRIR PLUS}

Convoitée par l'ensemble des services de l'Institut et de l'Ambassade pour l'organisation d'événements (conférences, concerts, cocktails...), la bibliothèque, et en particulier sa salle de lecture Art Déco, se devait d'avoir des plages de fermeture fixes pendant lesquelles accueillir ces manifestations. À mon arrivée, face à l'inflation des demandes en interne, j'ai même choisi à contrecœur de fermer une heure plus tôt le jeudi soir et de compenser en fermant une heure plus tard le samedi, afin de sanctuariser le jeudi pour l'organisation d'événements en soirée à la médiathèque. Ouvrir plus sur le papier, cela aurait voulu dire fermer plus souvent de manière « exceptionnelle », une source potentielle de mécontentement du public bien connue des professionnels.

Il est souhaitable, même lorsque les moyens diminuent, d'essayer de maintenir l'ouverture de sa bibliothèque car diminuer les horaires c'est franchir une ligne qui ne se traverse souvent qu'une fois. L'absence de volonté politique forte, des habitudes professionnelles qui ont la vie dure, des moyens limités et un contexte peu favorable : c'est ce que j'ai connu à Londres, c'est ce que connaissent un très grand nombre de bibliothèques 
en France et c'est souvent ce qui justifie qu'elles n'ouvrent pas davantage. Ce n'est pourtant pas une fatalité.

\section{CONTOURNER LE LIEU ET RATTRAPER LE TEMPS : RÉALITÉS DIVERSES}

Les solutions que je trouvais au casse-tête londonien peuvent s'appliquer à bon nombre de bibliothèques françaises. Si elles ne suffisent pas toujours à augmenter les horaires d'ouverture à court terme, au moins :

- améliorent-elles la qualité du service rendu ;

- préparent-elles le terrain pour de futurs horaires plus larges : un public content c'est un public qui fera pression, ne serait-ce que par le nombre, pour ouvrir plus ;

- en complément ou en substitution, on peut aussi aller chercher la solution aux problèmes d'ouverture sur d'autres terrains classiques, les ouvertures partielles ou spécifiques ; plus modernes, les services en ligne ; encore innovantes et pionnières, les bibliothèques numériques.

\section{RENDRE LA BIBLIOTHĖQUE DIGNE D'ÊTRE OUVERTE}

La priorité à Londres pour optimiser les horaires d'ouverture existants, c'était d'améliorer la qualité du service rendu donc d'augmenter le temps humain dévolu à ce dernier. N'ayant pas la possibilité d'embaucher du personnel, je décidais d'augmenter le nombre de tâches réalisées par chacun et pour cela d'automatiser, d'externaliser ou de supprimer les tâches jugées non prioritaires ou plus exactement, pouvant être assumées par d'autres sans perte de valeur ajoutée pour la bibliothèque. J'installais des automates de prêt et retour en nombre suffisant pour pouvoir absorber la totalité des transactions. La création d'un module d'inscription en ligne avec envoi des cartes au domicile, ajoutée à la possibilité de prolonger, réserver ses documents ou consulter son compte en ligne, permit de désengorger en partie la banque d'informations. Une ré-informatisation nous a permis de mettre en place un logiciel avec saisie automatique des notices 
à partir de l'ISBN. L'équipement des documents se fait désormais à l'aide de couvertures amovibles plus rapides à installer. Nous avons décidé de ne travailler qu'avec la librairie voisine de l'Institut pour recevoir les livres commandés très rapidement, ce qui introduit une habitude d'accélération du circuit du document. Nous expérimentons aussi l'externalisation des acquisitions ce qui accélère le circuit des commandes. J'ai également réduit le volume des collections et revu la politique documentaire de la bibliothèque pour passer à des acquisitions ciblées sur certains secteurs. Libérée de nombreuses tâches, l'équipe a pu davantage se consacrer à son public, non pas en ouvrant plus dans un premier temps, mais en proposant tout de même plus de services et d'ouvertures spécifiques, notamment la mise en place d'une politique d'animations et d'accueils scolaires plusieurs fois par semaine qui n'existaient pas jusque-là.

\section{CRÉER UN CONTEXTE FAVORABLE À UN FUTUR ÉLARGISSEMENT DES HORAIRES}

Une fois le quotidien amélioré et le minimum assuré, il fallait penser à l'avenir. Pour envisager d'ouvrir plus il fallait rendre les locaux plus fonctionnels. Pour être sûr de pouvoir ouvrir plus, il fallait faire en sorte que le public fasse pression pour. En bref, il fallait rénover. Comme il n'y avait aucun espoir du côté des budgets publics, je suis allée chercher du côté du privé : le Trust des Amis de l'Institut français accepta de financer un projet de rénovation complète des locaux. 1,5 million de livres sterling furent levés auprès de particuliers et d'entreprises pour réaliser des travaux divisés en trois tranches (salle de lecture Art-Déco, bibliothèque jeunesse, espaces adultes) afin de n'être jamais totalement fermés au public. L'objectif de ces travaux était d'aller plus avant dans la rationalisation des pratiques professionnelles:

- installer des points d'information un peu partout afin que le personnel puisse informer au mieux le public et en un temps record ;

- installer un mobilier plus bas, permettant d'avoir une meilleure visibilité sur l'ensemble de l'espace ; 
- " zoner » les publics, à l'image de ce qui se fait dans les Learning Centres afin de regrouper les publics qui étudient sur place et qui, a priori, nécessitent moins la présence de personnel ;

- à l'inverse, regrouper dans des zones « bruyantes » les publics en quête d'information ou de discussions libres.

En parallèle, j'ai commencé à préparer mon équipe à réaliser davantage de service public, tout d'abord en introduisant un système de renfort (personnel doublé pendant ces plages horaires) à l'ouverture et à la fermeture des deux bibliothèques adultes et jeunesse puis en créant un nouveau point d'information doté d'un personnel supplémentaire pendant les heures de pic d'affluence en bibliothèque adultes. Toute offre créant la demande, ce qui n'était qu'une expérience est maintenant devenu, aux yeux de tous, une absolue nécessité. L'équipe consacre désormais $50 \%$ de son temps à l'accueil, une évolution qui a été facilitée par le renouvellement récent d'une partie de l'équipe (ce quota de service public a été inclus dès le début dans la fiche de poste des nouvelles recrues). Le succès de la médiathèque rénovée poussera sans aucun doute la direction de l'Institut à augmenter les heures de vacation de la bibliothèque et par extension les horaires d'ouverture, en particulier en bibliothèque enfants dont l'activité a atteint un plafond indépassable attribué à ses faibles horaires $^{5}$. L'automatisation permettra même d'aller encore plus loin et, qui sait, un jour, d'ouvrir en nocturne en plaçant uniquement un surveillant dans la bibliothèque : le public pourrait consulter les documents sur place et/ou emprunter via les automates.

\section{OUVRIR UN LIEU VIRTUEL 7 J/7 ET 24 H/24}

À mon arrivée à Londres, l'Institut français venait d'inaugurer une plateforme numérique : «Culturetheque.org.uk ». Absolument pionnier, ce site Web gratuit et accessible à tous, entièrement géré par les bibliothécaires, offrait une collection numérique de 5000 documents (e-books, vidéos, podcasts...) et permettait ainsi à la bibliothèque d'exercer réellement

5. De 2010 à 2013, tous les indicateurs de la médiathèque de l'Institut français de Londres ont augmenté, à l'exception des visites et des inscriptions en bibliothèque enfants. 
sa mission en étant accessible à l'ensemble des résidents du RoyaumeUni. Je m'attelais donc à la promotion et à l'enrichissement de "Culturethèque » qui, dotée désormais de plus de 25000 documents et fière de ses 9000 inscrits (là où la bibliothèque en compte 3 000), a permis de dépasser de nombreuses contraintes liées au lieu physique en attendant les travaux de ce dernier, de redorer son image et, en quelque sorte, de l'ouvrir davantage.

La question des horaires d'ouverture fait mal car elle reflète l'organisation de la bibliothèque et, au-delà, l'harmonie qui a été trouvée entre travail interne et service public, deux pôles souvent opposés de notre activité, à tort selon moi car ils ne font qu'un.

Ouvrir, même avec des moyens, nécessite une organisation et une prévisualisation hors pair. Ouvrir plus, sans moyens significatifs, nécessite une rationalisation parfaite qui peut faire peur alors qu'en réalité elle vise à se concentrer sur l'humain et sur la valeur qu'il apporte au service rendu. Il y a toujours des solutions pour ouvrir davantage si l'amélioration du service est l'objectif permanent du chef de service.

Ouvrir plus, même sans prévoir de service supplémentaire, c'est déjà un plus dans la qualité de l'offre de la bibliothèque et opposer systématiquement quantité des horaires et qualité du service rendu est selon moi un autre faux débat. Même sans l'avouer, le public sera toujours intéressé par une ouverture plus large. C'est à la bibliothèque de surprendre son public, d'être là où il ne l'attend pas et de le pousser à être de plus en plus exigeant vis-à-vis d'elle. 


\section{2}

HEUREUX ÉTUDIANTS

DE NANTES! LE SCD DE L'UNIVERSITÉ DE NANTES

par Hélène

Grognet et

Emmanuelle

Paulet-

Grandguillot

Le SCD comprend 7 BU. 5 sont localisées sur les différents campus nantais : Lettres, Sciences humaines et sociales ; Droit, Économie et Gestion ; Sciences, Techniques et STAPS ; Technologies ; Santé. Les sites délocalisés de l'université, La Roche-sur-Yon et Saint-Nazaire bénéficient également d'une bibliothèque universitaire.

\section{TABLEAU QUELQUES CHIfFRES SUR LA BU SANTÉ (2012)}

\begin{tabular}{|c|c|c|c|}
\hline Surface SHON & Places assises & $\begin{array}{c}\text { Ouverture } \\
\text { hebdomadaire }\end{array}$ & $\begin{array}{c}\text { Nombre annuel } \\
\text { d'entrées }\end{array}$ \\
\hline $6400 \mathrm{~m}^{2}$ & 633 & $104 \mathrm{~h} 30$ & 536277 \\
\hline
\end{tabular}

\section{NOCTAMBU}

Le SCD bénéficie du label NoctamBU*, label du ministère de l'Enseignement supérieur et de la Recherche attribué aux bibliothèques universitaires ouvrant plus de 65 heures par semaine, dans le cadre du Plan Bibliothèques universitaires lancé le 17 février 2010 par Valérie Pécresse, alors ministre.

\section{LE FONCTIONNEMENT : 2014}

\section{Quoi ?}

La BU Santé est située aux étages 4, 5 et 6 du nouveau bâtiment de l'UFR de Pharmacie. Sur les plages d'horaires élargis, sont ouverts les niveaux 4 et une partie de l'étage 6.390 places de travail sont disponibles, dont 90 équipées d'ordinateurs, et dont 44 dans 5 salles de travail en groupe. 
Sont également accessibles 2 photocopieurs-numériseurs. Les salles ont des accès wifi. Les ressources électroniques sont évidemment disponibles. En revanche, il n'y a pas d'accès aux collections physiques, situées au niveau 5 et sur une partie du niveau 6 fermés en NoctamBU. Très vite, les étudiants ont pris l'habitude de prendre avant $19 \mathrm{~h}$ sur les rayonnages des salles fermées en NoctamBU les ouvrages dont ils avaient besoin pour leurs révisions (notamment les ouvrages exclus du prêt).

\section{Quand ?}

\section{TABLEAU LES HORAIRES NOCTAMBU (SAUF LES JOURS FÉRIÉS)}

\begin{tabular}{|c|c|c|c|c|c|c|c|}
\hline & Lundi & Mardi & Mercredi & Jeudi & Vendredi & Samedi & Dimanche \\
\hline $\begin{array}{c}\text { Horaires } \\
\text { BU }\end{array}$ & $\begin{array}{c}8 \mathrm{~h} 30- \\
19 \mathrm{~h}\end{array}$ & $\begin{array}{c}8 \mathrm{~h} 30- \\
19 \mathrm{~h}\end{array}$ & $\begin{array}{c}8 \mathrm{~h} 30- \\
19 \mathrm{~h}\end{array}$ & $\begin{array}{c}8 \mathrm{~h} 30- \\
19 \mathrm{~h}\end{array}$ & $\begin{array}{c}8 \mathrm{~h} 30- \\
19 \mathrm{~h}\end{array}$ & $\begin{array}{c}8 \mathrm{~h} 30- \\
13 \mathrm{~h}\end{array}$ & \\
\hline $\begin{array}{l}\text { Horaires } \\
\text { NoctamBU }\end{array}$ & $\begin{array}{c}19 \mathrm{~h}- \\
23 \mathrm{~h} 30\end{array}$ & $\begin{array}{c}19 \mathrm{~h}- \\
23 \mathrm{~h} 30\end{array}$ & $\begin{array}{c}19 \mathrm{~h}- \\
23 \mathrm{~h} 30\end{array}$ & $\begin{array}{c}19 \mathrm{~h}- \\
23 \mathrm{~h} 30\end{array}$ & $\begin{array}{c}19 \text { h- } \\
23 \text { h } 30\end{array}$ & $\begin{array}{c}13 \mathrm{~h}- \\
23 \mathrm{~h} 30\end{array}$ & $\begin{array}{c}9 \text { h- } \\
23 \text { h } 30\end{array}$ \\
\hline
\end{tabular}

\section{Qui ?}

Le service NoctamBU est ouvert à tous les lecteurs (étudiants, enseignants et enseignants-chercheurs, lecteurs extérieurs) inscrits au SCD, et non pas aux seuls étudiants du Pôle Santé. Pour accéder au service, les usagers doivent réserver leur place via un logiciel spécifique, accessible sur Internet, sur place, ou encore par téléphone ${ }^{1}$. L'annulation est simple, avec les mêmes outils. Pour valider sa réservation, l'usager dépose sa carte d'étudiant ou de lecteur, et se voit remettre un badge magnétique qui lui permet ensuite d'entrer et sortir du bâtiment à sa guise. Ce badge n'est actif que pendant la journée de réservation, y compris le week-end. Il doit être restitué au bureau d'accueil de la bibliothèque en partant. L'accès se fait par une entrée latérale du bâtiment qui conduit à un ascenseur ne desservant que le $4^{\mathrm{e}}$ étage, respectant ainsi la fermeture des espaces de l'UFR.

1. Cette possibilité a été mise en place pour permettre aux étudiants qui n'ont pas accès à Internet en dehors de la BU de pouvoir réserver sans venir sur place. Elle est surtout utile aux étudiants de nationalité étrangère. 


\section{comment?}

La surveillance des salles et le contrôle des réservations sont assurés par deux tuteurs-étudiants (formés par les personnels de la BU), avec le soutien de deux agents extérieurs chargés de la sécurité du bâtiment de l'UFR de pharmacie. 10 étudiants sont nécessaires pour couvrir toute l'amplitude horaire, avec des contrats prévoyant des horaires hebdomadaires de $9 \mathrm{~h}$ 30, $10 \mathrm{~h}$ et $11 \mathrm{~h}$ par semaine, selon les tranches proposées. Les étudiants travaillent en binômes et doivent assurer une plage en soirée en semaine, et une autre le week-end.

\section{ENCADRÉ LES MISSIONS DES TUTEURS}

- assurer la liaison avec le personnel de la BU (pendant la demi-heure de travail commune de 18 h 30 à 19 h ou par le biais de la main courante) ;

- relever le compteur des entrées (le dimanche matin uniquement) ;

- contrôler les réservations des usagers et leur délivrer un badge magnétique en échange de leur carte d'étudiant ;

- assurer un dépannage de premier niveau sur les photocopieurs ;
- effectuer des rondes de surveillance dans les salles (respect de l'interdiction de manger, de boire...) ;

- à la fermeture, rassembler sur un chariot les ouvrages laissés par les étudiants sur les tables ;

- participer à l'évacuation en cas d'incident majeur. L'ingénieur sécurité $\mathrm{du}$ campus organise pour les tuteurs une formation guide-files/serre-files et un exercice d'évacuation, en septembre.

\section{LA PRÉPARATION : 2008-2011}

En 2008, sur demande du ministère de l'Enseignement supérieur et de la Recherche et du président de l'université, le SCD a étudié, en concertation avec le personnel, la possibilité d'étendre ses horaires d'ouverture. Très vite, il est apparu pertinent de concentrer les efforts sur la nouvelle BU Santé, dont la réalisation était imminente, située en centre-ville, où une très forte demande des étudiants de médecine s'exprimait. D'autre part, la conception même des espaces permettait d'isoler les collections papier de salles pouvant être ouvertes plus largement. 


\section{ENCADRÉ LES MISSIONS DES AGENTS DE SÉCURITÉ}

- contrôler les badges et les cartes d'étudiants ;

- soutenir les tuteurs dans leurs relations avec les usagers (en cas de litiges, de tensions...) ;

- assurer des rondes dans les espaces ouverts (une ronde de vérification systématique des badges délivrés aux étudiants ${ }^{1}$ une heure après l'ouverture du service de retrait de ceux-ci,

1. Elle permet de s'assurer que toutes les personnes présentes dans la bibliothèque ont bien réservé une place et validé leur réservation auprès des tuteurs. une ronde de surveillance des salles toutes les heures et demie) ;

- gérer les incidents, éventuellement avec l'aide du PC Sécurité chargé des 2 bâtiments, Médecine et Pharmacie ;

- porter secours aux personnes ;

- assurer les ouvertures et les fermetures, assister les tuteurs dans l'évacuation des usagers au moment de la fermeture ;

- encadrer l'évacuation du bâtiment en cas d'incident majeur et prévenir les secours.

Le projet a été labellisé NoctamBU par courrier du 10 mars 2011 du ministère de l'Enseignement supérieur et de la Recherche et l'université a reçu jusqu'en 2013 une subvention de $150000 €$, sur les $165000 €$ qui étaient sollicités (rémunération des tuteurs-étudiants et estimation des coûts gardiennage/sécurité).

Après validation par le doyen de l'UFR de Médecine et le président de l'université, le projet a été présenté en Comité technique paritaire du 17 mai 2011. Les débats ont tourné autour de l'opposition à une ouverture le dimanche, et autour de la vigilance à mettre en œuvre sur les questions de sécurité. Enfin, un point a été mis en avant : ce n'est pas une bibliothèque qui est ouverte en NoctamBU, mais des espaces avec des places assises. Le projet a été également présenté en Conseil de la documentation le 2 avril 2010, et aux représentants du personnel au sein du SCD le 29 avril 2011.

La Commission pédagogique des étudiants de Médecine a été consultée tout au long du chantier de la bibliothèque, aussi bien sur les espaces, la répartition des collections, les services proposés... que sur les horaires d'ouverture. Les horaires du service NoctamBU ont été calés sur ceux qui 
étaient de mise à la Faculté de Médecine, où le Doyen de l'UFR de Médecine mettait à la disposition de ses étudiants un amphithéâtre et la salle des assemblées ; le contrôle des accès était alors assuré par les agents du PC Sécurité.

La nouvelle bibliothèque ouvre ses portes le 16 août 2011. Outre une vue superbe sur la Loire, ses deux grands plateaux aux murs blancs, rehaussés par des moquettes rouges et orange offrent une ambiance sereine de travail immédiatement appréciée par le public. Entre 2011 et 2012, le nombre d'entrées va augmenter de $45 \%$ (de 370333 à 536 277), le nombre de lecteurs inscrits de $32 \%$ (de 3338 à 4 399), et le nombre d'inscriptions payantes (lecteurs extérieurs à l'université) sera multiplié par 6 (de 42 à 266).

\section{L'ANNÉE TEST : 2011-2012}

Les horaires NoctamBU ont débuté le 5 septembre 2011. Volontairement, il n'y a pas eu de communication spécifique. La priorité était mise à ce moment sur les 50 ans de l'université, et le SCD souhaitait tester " grandeur nature » le dispositif, notamment le logiciel de réservation élaboré en interne.

Le service rencontre assez vite un franc succès. Sur l'année 2011-2012, 62466 entrées ont été enregistrées ${ }^{2}$. La proportion des usagers hors université a été de $7 \%$. La répartition par ensemble disciplinaire (qui inclut les usagers extérieurs à l'université) est la suivante :

- Santé : $84 \%$;

- Droit, Économie, Gestion : 7 \% ;

- Sciences : $6 \%$;

- Lettres et Sciences humaines : $3 \%$.

Au sein des étudiants de Santé, deux groupes ont constitué rapidement, et logiquement, les « noyaux durs » en termes de nombre et de régularité de fréquentation : la première année commune aux études de Santé et les

2. Les statistiques concernent les entrées réelles, (c'est-à-dire le nombre de badges remis), et non les réservations. 
étudiants de $6^{\mathrm{e}}$ année de Médecine préparant l'Examen classant national (ECN). Les écoles paramédicales extérieures à l'université ont été également bien présentes.

Travaillant à partir de leurs documents ou de documents empruntés à la BU, les étudiants n'ont pas exprimé de demande particulière. Ceux de médecine ont vite compris qu'ils pouvaient utiliser sur place sans les emprunter les ouvrages de préparation aux concours. Le travail de rangement du matin a ainsi singulièrement augmenté.

Le système de réservation s'est révélé efficace, mais certains lecteurs n'honoraient pas leur réservation ${ }^{3}$. Le SCD a donc mis en place dès mars 2012 un système de pénalité : l'usager qui ne vient pas ne peut pas s'inscrire le jour suivant. Approuvé en Conseil de la documentation en novembre 2011, ce système a permis de ramener le nombre de réservations non-honorées à une douzaine par jour.

En terme de charge de travail, le pilotage a été assuré par le responsable de la BU Santé (parti à la retraite à l'été 2012) et la directrice. Deux autres services transversaux du SCD ont également été largement sollicités : le service informatique pour le logiciel de réservation et les services administratifs pour la gestion des 10 contrats. Les agents de la BU Santé ont assumé la communication des informations auprès du public.

\section{LA CONFIRMATION DU SUCCÈS : 2012-2013}

Le départ de 4 collègues dont le responsable (tous remplacés), le changement de responsable de l'équipe accueil-magasinage, suscitent une réorganisation des missions et des activités de chacun. NoctamBU repose alors sur 8 collègues : recrutement et gestion des 10 tuteurs par le responsable, formation par les cadres A. Le suivi quotidien du service (gestion des pénalités appliquées aux usagers, suivi de la main courante) est confié à 6 collègues volontaires. Une secrétaire se charge plus spécifiquement de la gestion des badges et une correspondante informatique du logiciel. L'ensemble de l'équipe demeure sollicité, pour assurer le lien à 18 h 30 avec les tuteurs, répondre aux nombreuses questions des usagers sur le

3. Jusqu'à 100 réservations non validées sur une seule journée. 
service, inscrire les lecteurs extérieurs, veiller par roulement au suivi du service le samedi matin.

NoctamBU fait cette fois l'objet d'une campagne d'affichage ; il est également présenté dans l'ensemble des BU lors des formations aux usagers. Le nombre d'entrées passe à 78 460, soit une augmentation de $25 \%$.

En termes de composition des publics, la part des usagers extérieurs à l'université double pratiquement par rapport à l'année précédente, passant de 7 à $13 \%$. Il s'agit essentiellement d'étudiants en filière Santé : instituts de soins infirmiers, écoles de kinésithérapie, instituts d'ostéopathie, école de podologie. Deux nouvelles catégories apparaissent : les élèves des classes préparatoires aux grandes écoles et les demandeurs d'emplois.

Cependant, les étudiants de l'université restent les plus nombreux à fréquenter NoctamBU : $87 \%$ des entrées (soit 68 121). La répartition par ensemble disciplinaire évolue peu, même si l'on constate une légère baisse du Pôle Santé au profit des étudiants de Droit, Économie et Gestion et de Lettres et Langages.

Le risque d'être dépassés par ce succès s'est très vite fait sentir. Il suscite deux réflexions.

L'une porte sur l'augmentation du nombre de places. Après étude des plans, et validation de l'implantation retenue par l'ingénieur sécurité du campus, il s'avère possible d'ajouter une cinquantaine de places. Malheureusement, ce projet ne parvient pas à trouver son financement (40 000 euros TTC) $)^{4}$.

L'autre vise le système de réservations, celles-ci étant complètement saturées. En outre, les pénalités engendrent une charge de travail accrue pour les personnels, ainsi qu'un fort mécontentement chez les usagers. Enfin, l'interface est peu lisible avec un ordiphone.

Un nouveau système est mis au point, qui évite les pénalités. La réservation aura une durée limitée : si elle n'est pas honorée par l'étudiant, passée cette durée, la place réservée retournera dans le pot commun des places disponibles. Une application mobile est développée, toujours en interne.

4. Pour l'ajout au total de 76 places sur l'ensemble de la BU Santé, dont 50 en NoctamBU. 


\section{L'AMÉLIORATION DES FLUX : 2013-2014}

Avec l'arrêt des subventions de l'État, l'université décide de prendre à sa charge l'ensemble des crédits nécessaires, malgré une situation financière difficile.

Mis en place en septembre 2013, le nouveau système de réservation a amélioré la gestion des flux, en empêchant les étudiants de bloquer une place pendant plusieurs heures, en mettant fin à la précipitation dès l'ouverture des réservations. D'autres améliorations ont été apportées : suivi de la gestion des badges, main courante informatisée. De ce fait, la charge de travail liée à la gestion de NoctamBU a pu être resserrée sur 4 personnes. En revanche, le rangement des ouvrages ne fait que croître : sur l'année 2013-2014, l'augmentation du nombre de documents consultés sur place pendant les mois de septembre à janvier a été de $12 \%$ par rapport à l'année précédente (de 4225 à 4 762).

Mécaniquement, le nouveau système de réservation permet à plus d'usagers de profiter du service. On peut d'ores et déjà mesurer une augmentation de $13 \%$ du nombre des entrées sur les mois de septembre à février (55 329 entrées entre septembre 2013 et février 2014, contre 48993 sur la même période en 2012-2013). On constate aussi une diversification accrue des publics : progression des étudiants des autres universités (de 82 à 267 entrées), des lycéens (de 14 à 69 entrées), des demandeurs d'emplois (de 127 à 238 entrées), des élèves de l'École de sages-femmes (de 29 à 112 entrées), du STAPS (de 106 à 175 entrées).

À la demande des étudiants (sans restaurant universitaire le soir, ils pique-niquaient dans l'entrée du parking), la cafétéria du bâtiment reste maintenant ouverte jusqu'à $20 \mathrm{~h}$.

\section{BILAN GÉNÉRAL}

Le succès public est indéniable. Cette ouverture élargie répond à un véritable besoin : des étudiants au premier chef, mais aussi d'autres populations, comme les personnes exerçant une activité professionnelle tout en préparant un diplôme ou un concours. Tous recherchent des espaces de 
travail pour réviser. Ouverte de 8 h 30 à 23 h 30 tous les jours de la semaine, la bibliothèque devient pour les étudiants un lieu de vie très apprécié. NoctamBU à Nantes n'offre pas tous les services d'une bibliothèque, mais s'appuie très fortement sur un travail quotidien mené par les personnels. La charge de travail avait été sous-estimée, même s'il a été possible de l'assumer.

NoctamBU est aussi un vrai outil de promotion. Nombre d'étudiants qui s'inscrivent « pour NoctamBU » découvrent alors qu'au-delà d'horaires larges, ils auront accès à tous les autres services offerts par les BU.

\section{PERSPECTIVES}

En termes d'évolution, le SCD porte deux projets.

L'un, déjà cité, concerne l'augmentation du nombre de places assises, dans la BU Santé, en horaires élargis.

L'autre est relatif à une opération immobilière qui pourrait être financée dans le cadre du Contrat de plan État-région (CPER) 2015-2020. Il s'agit de la réhabilitation et du réaménagement de 3 BU (Sciences, Lettres et Droit), permettant d'augmenter le nombre de places assises, et d'organiser la BU Droit avec une partie du rez-de-chaussée uniquement consacrée à cellesci. Dans ce cadre, la réflexion sur une meilleure adéquation des horaires d'ouverture par rapport aux besoins constatés serait facilitée, sachant que la situation très excentrée du campus ne permettra pas une offre aussi conséquente qu'en centre-ville : à quoi bon ouvrir, quand les étudiants ne trouvent pas aussi sur place de quoi se restaurer, et des facilités de transports, dans un campus totalement ouvert qui génère un sentiment d'insécurité manifeste?

Ouvrir largement, oui : mais au bon moment et au bon endroit. 


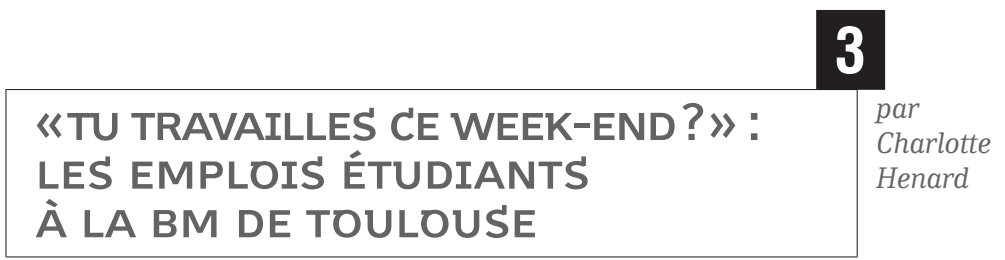

La médiathèque José Cabanis a ouvert ses portes en mai 2004, et constitue une des entités de la Bibliothèque municipale à vocation régionale (BMVR) de Toulouse, avec la Bibliothèque d'étude et du patrimoine (BEP) ${ }^{1}$ et les bibliothèques de quartier. Situé dans la ville, et profitant d'une position privilégiée, proche de la gare, le projet visait à accueillir de nombreux usagers et, ce, sur une large amplitude horaire. Le succès de la médiathèque, non démenti jusqu'à ce jour, alors qu'elle accueille quotidiennement plus de 3000 personnes, tient aussi à son ouverture dominicale. L'ouverture du dimanche était une composante importante du projet, voire même un préalable, et explique la présence d'équipe d'étudiants en renfort des équipes de titulaires.

Pour permettre une ouverture dominicale tout au long de l'année (à l'exception des mois de juillet et août pendant lesquels la médiathèque accueille le public du mardi au samedi), il a été décidé de faire appel à des emplois dits " étudiants » qui viendront soutenir les équipes pour le travail du week-end. Ce choix n'a pas fait l'objet de longues discussions entre la direction et la tutelle : il était une composante du projet dès le départ. De même que les équipes recrutées pour l'établissement s'engageaient à travailler le dimanche, la présence d'étudiants en soutien du personnel titulaire était un prérequis à l'ouverture. Si les premiers jours de la médiathèque ont été le théâtre d'un conflit social d'une assez grande ampleur, les grèves n'ont jamais eu pour motif la présence d'étudiants. On dira même que le cas des non-titulaires (étudiants, contractuels...) a été intégré dans les revendications du personnel gréviste, sur des questions salariales notamment : la majoration des heures travaillées le dimanche

1. Ancienne bibliothèque municipale centrale de Toulouse, avant l'ouverture de la médiathèque José Cabanis. 
à l'ensemble du personnel - titulaires et étudiants - a été, entre autres, demandée et obtenue.

Environ cinquante personnes sont nécessaires pour assurer l'accueil et le bon fonctionnement du service au public (pour une médiathèque de $13500 \mathrm{~m}^{2}$ ).

\section{DES TITULAIRES AVEC DES ÉTUDIANTS}

Pour les équipes de titulaires, l'emploi du temps est mensualisé sur 140 heures (ou 148 heures dans le choix d'un temps de travail hebdomadaire à 37 heures, choix bonifié par des jours de congés supplémentaires), du mardi au vendredi, à quoi se rajoutent un week-end travaillé par mois et un lundi travaillé par mois.

En 2004, chaque week-end, 26 titulaires sont présents, soutenus par une équipe de 26 étudiants (23 à partir de 2009). On dira donc que les besoins du service public, ainsi que l'emploi du temps déterminé pour les titulaires, rendent indispensable la présence d'étudiants pour ouvrir la médiathèque les samedis et dimanches.

Ainsi en 2004, 52 étudiants ont été recrutés à cette fin. En 2009, avec la mise en place de la RFID et du prêt automatisé, l'équipe des étudiants a été ramenée à 46. Pour l'ouverture du Grand $\mathrm{M}^{2}$ en 2012, une équipe de 10 étudiants a été recrutée en sus.

\section{DES ÉTUdIANTS}

\section{Quand?}

Les étudiants, prévus donc pour venir en renfort des équipes de titulaires, travaillent le samedi de 9 h 45 à 19 h 15 et le dimanche de 13 h 45 à $18 \mathrm{~h}$ 15. Ils effectuent un week-end sur deux, soit 26 heures par mois, et ce pendant la durée de leur contrat de septembre à fin juin. Ce fonctionnement n'a pas varié depuis dix ans.

2. La médiathèque Grand $\mathrm{M}$, inaugurée dans le quartier du Mirail en juin 2012, est ouverte 42 heures par semaine, dont 4 heures le dimanche, de 14 à $18 \mathrm{~h}$. 
Les étudiants sont parfois sollicités pour travailler durant les congés scolaires. La bibliothèque gère donc un crédit de vacations qui sont réparties tout au long de l'année. Ces vacations sont destinées uniquement à renforcer les équipes de la BMVR pendant les congés et ne sont jamais utilisées pour des remplacements. La bibliothèque doit donc répartir avec parcimonie ces contrats, en faisant un planning prévisionnel des besoins pour assurer le service au public. Cette gestion permet d'accorder des congés scolaires au personnel titulaire, sous réserve de $50 \%$ de présence des équipes pendant ces périodes. Les congés sont donc accordés sous réserve de nécessité de service public et les étudiants assurent le service jusqu'à $50 \%$ maximum de ce temps. Seuls les congés scolaires sont concernés. Dans ce cas, et selon les besoins évalués, les étudiants travaillent pendant la semaine et le weekend, selon un emploi du temps complexe, dans le respect de la législation sur le temps de travail. Durant les mois d'été, les contrats sont d'une durée d'un mois, et sont répartis en fonction des besoins.

Ce fonctionnement a été étendu à la médiathèque Grand $\mathrm{M}$, inaugurée en juin 2012, selon les mêmes modalités, car elle est aussi ouverte le dimanche. En dehors des périodes scolaires, la sollicitation d'étudiants sur le réseau des bibliothèques de quartier (fermées le dimanche) ne fait pas partie du dispositif, et reste exceptionnelle (quelques samedis pour la BEP, ou cas de congés de maladie imprévu par exemple, avec remplacement au pied levé par un étudiant).

\section{Pour quoi faire ?}

Depuis l'ouverture de Cabanis, les étudiants sont prévus pour assurer le service au public. Le week-end, les équipes de titulaires travaillent en front office toute la journée, tandis que durant la semaine les emplois du temps aménagent des plages de travail interne et de service au public, en alternance, répartis différemment selon les catégories ${ }^{3}$.

Les étudiants ont des tâches très définies, et qui ne varient pas d'un service à l'autre, ils doivent donc effectuer :

3. Pour le personnel titulaire, la répartition du service au public (front office) par catégorie pendant la semaine est la suivante : Catégorie C : 50 \%; Cadre B : $30 \%$; Cadre A : 15 à $20 \%$; Cadre A transversaux : $10 \%$; Cadre A $+: 2 \mathrm{~h}$ par semaine. 
- le prêt et le retour au rez-de-chaussée (puis retour seul et vérification de l'état des documents retournés dans la boîte à documents, après la mise en place de la RFID en 2009) ;

- l'accueil et le renseignement sur les pôles thématiques ;

- le rangement des collections.

\section{Quel recrutement ?}

Les conditions de recrutement ont considérablement évolué en dix ans. Les conditions d'accès en revanche n'ont pas varié : il faut être étudiant et avoir plus de 18 ans. Les candidatures des étudiants en documentation et bibliothéconomie sont étudiées en priorité, même si ce n'est pas un prérequis. Hormis cette préférence, aucune filière n'est privilégiée, et la diversité est recherchée, qu'il s'agisse de l'origine linguistique des étudiants ou de leurs études (du DUT au M2, en passant par Sciences Po ou des études d'infirmières...). Dans la ligne du travail d'inclusion entamé par la bibliothèque, bien connue pour son travail autour du handicap, le service Accueil s'efforce de recruter des étudiants maîtrisant la langue des signes. $\mathrm{Au}$ début, pour la constitution de l'équipe des étudiants, le simple envoi d'un CV (arrivé au bon moment) ou une recommandation pouvaient suffire pour une embauche. Un appel téléphonique, une rapide évaluation et l'étudiant était recruté. Les contrats n'étaient pas limités dans le temps, ainsi certains ont travaillé pendant plusieurs années à la médiathèque. Depuis 2009, des modalités plus strictes ont été établies en accord avec la tutelle :

- une date limite (en mars) pour l'envoi d'un CV et d'une lettre de motivation ;

- un entretien de motivation (en mai). Cet entretien a pour but d'évaluer le candidat : il est interrogé sur sa connaissance de la bibliothèque (être abonné ou fréquentant de la médiathèque est conseillé...), ses aptitudes relationnelles et son sens du service public. Pour certains, c'est le premier entretien de leur vie, et c'est un bon entraînement ; 
- un contrat limité à deux ans avec un recrutement en septembre.

\section{Le rôle du service Accueil}

C'est le service Accueil qui gère l'équipe des étudiants. Il effectue les recrutements, la répartition dans les services en fonction des études poursuivies ; il gère les feuilles de présence, les éventuelles absences (l'université programmant parfois des partiels le samedi par exemple, la bibliothèque s'efforce alors de remplacer l'étudiant pour le libérer), détermine les besoins en vacations durant les congés scolaires, et assure le lien avec le service de gestion des ressources humaines pour la paie et les tickets restaurants. Le service Accueil est l'interlocuteur principal des étudiants dans ses relations avec la bibliothèque, et est chargé de l'information sur les évolutions du service (le plus souvent par email). Chaque année, les responsables de service remplissent une évaluation de chaque étudiant, qui conditionne la prolongation des contrats (ou pas) à la médiathèque. Sont évalués : la relation avec le public, le relationnel avec les équipes, la ponctualité, les capacités d'adaptation et la capacité à mobiliser ses connaissances. En cas de problème manifeste, le service Accueil pourra décider du transfert vers un autre service ou du non-renouvellement du contrat, selon les cas.

\section{LA FORMATION DES ÉTUDIANTS}

Les étudiants recrutés sont dotés de qualités et d'aptitudes pour le poste : à leur arrivée, ils ne sont pas qualifiés. Il revient donc à l'établissement de les former. Pour ce faire, une journée complète rémunérée est coassurée par le service Accueil et le service qui gère le SIGB. Ainsi, le maniement du logiciel pour le prêt-retour et la recherche documentaire leur est enseigné, sous la forme d'une présentation assortie de mises en situation. Les procédures et des consignes de tenue en situation d'accueil complètent ce dispositif. 


\section{L'INTÉGRATION DES ÉTUDIANTS DANS LES ÉQUIPES}

En fonction des services auxquels ils sont affectés, leurs tâches peuvent être variables. Si le principe de leur présence est acquis, les modalités de leur intégration sont dépendantes des équipes auxquelles ils sont intégrés. Ainsi avec certains pôles, ils peuvent être cantonnés aux tâches strictement déterminées. Dans d'autres équipes, ils sont considérés comme des collègues à part entière et, de ce fait, effectuent les mêmes tâches que les titulaires. Ainsi, au fil du temps, et en fonction de leurs qualités, ils peuvent effectuer des tâches à plus forte valeur ajoutée : sélection de documents pour les présentoirs, vérifications et recherche de nouvelles éditions dans le cas du désherbage, vérification de listings, etc. Les étudiants ne s'y trompent pas : ces différences de traitement les poussent à préférer des services (et des équipes) à d'autres...

En dix ans, 11 étudiants ont été « stagiairisés » et 6 ont été embauchés en contrat à durée déterminée. L'un d'entre eux est devenu adjoint au maire de Toulouse, délégué à la médiathèque, bibliothèques et documentation, de 2008 à 2014.

\section{QUELS BÉNÉFICES ?}

\section{POUR LES ÉTUDIANTS}

Les avantages sont multiples et de différentes natures :

- une rémunération pour subvenir (en totalité ou en partie) à leurs besoins, et poursuivre ainsi leurs études ;

- un emploi aux horaires compatibles avec les heures de cours. Il permet aux étudiants de poursuivre leurs études sans manquer leurs cours. De plus, le travail du week-end est cumulable avec d'autres emplois plus aléatoires ou épisodiques (baby-sitting, cours de soutien, accompagnement...) ;

- l'adaptation à l'emploi. Cette fonction est non négligeable pour de nombreux étudiants. Il s'agit pour eux, le plus souvent, d'un premier emploi, où l'apprentissage de règles du travail est une 
découverte : comment se comporter, se conformer au rythme et aux horaires, s'adapter aux procédures et aux us et coutumes de la bibliothèque, découvrir les relations professionnelles ;

- un emploi à caractère culturel et social : assez unanimement, ce « job » en bibliothèque est considéré comme une expérience « intéressante », voir « enrichissante » par tous les anciens étudiants. L'accès aux multiples ressources proposées par la médiathèque est incontestablement un avantage pour les étudiants qui, pour la plupart, sont aussi des usagers, qu'ils s'agissent de documents pour leurs études ou leurs loisirs ;

- une première expérience professionnalisante pour les étudiants en métiers du livre et en documentation, avec la possibilité de la valoriser sur son CV et de faire référence à cet emploi lors des entretiens de recrutement ultérieurs. C'est un excellent moyen de "mettre le pied » dans les bibliothèques, et pour certains, un moyen de se découvrir une « vocation » et un goût réel pour le service public ;

- enfin, même si ce n'est pas un objectif, le travail du week-end est un excellent moyen pour se forger un réseau et se faire des amis. De nombreux étudiants, une fois fini leur contrat, sont restés en contact avec leurs co-équipiers, qu'ils soient étudiants ou titulaires...

\section{POUR LE PERSONNEL}

- Un emploi du temps régulier (voir plus haut) et, contrairement à la majeure partie de leurs collègues en France, la possibilité de bénéficier de 3 samedis libérés sur 4, tout en offrant des horaires élargis au dimanche à la population toulousaine et d'ailleurs ;

- un soutien pour toutes les tâches matérielles (retours, rangement...), par un étalement de la charge sur le plus grand nombre ; 
- un regard " extérieur de l'intérieur » porté sur les pratiques professionnelles. C'est un des apports important du mixage au sein des équipes : par leurs questions parfois naïves, parfois dérangeantes, souvent pertinentes, les étudiants poussent les professionnels à réinterroger des pratiques ou des habitudes. Ainsi, des équipes auront fait évoluer leurs services, grâce aux questionnements des étudiants (changement de signalétique, évolution de la cotation, changement de vocabulaire, clarification de certains process...), pour une meilleure prise en compte des attentes du public ;

- des apports et un brassage culturel : la fréquentation des étudiants, jeunes adultes, permet de connaître leurs centres d'intérêts et d'avoir des échanges avec eux sur leurs pratiques culturelles, leur façon de "voir les choses " sur la collection et les services. Ce contact avec le néophyte est précieux pour les professionnels : ainsi, on aura commandé des titres sur la recommandation de tel ou tel amateur de musique ou de littérature, ou modifié la signalétique pour mieux orienter les usagers... De tels apports sont fondamentaux, et réciproques, même s'ils sont difficilement évaluables quantitativement.

\section{POUR LES USAGERS}

Cet aspect est lui aussi difficilement quantifiable, mais les apports sont hautement probables. On constate notamment que certains usagers choisissent leur interlocuteur lors de leurs opérations à la médiathèque. Ils apprécient (ou pas) de s'adresser aux étudiants, sans doute par reconnaissance de "génération » ou dans un désir d'une relation qu'ils perçoivent comme plus " naturelle ", ou peut-être de nature moins " administrative ». Par leur positionnement et leur jeunesse, les étudiants ont des relations différentes avec le public.

On distingue d'ailleurs deux types d'interaction :

- au poste de retour, où l'usager s'adressera volontiers à l'étudiant, par préférence sociale ou pour rendre des documents 
endommagés par exemple (par désir de passer entre les mailles du filet ?) ;

- dans les pôles documentaires, la recherche de documents ou d'idées de lecture se double d'une relation où la sympathie et les affinités jouent leur rôle.

Ainsi, des recrutements multiculturels ont été mis en place depuis 2009, par le service Accueil, qui permettent de proposer des interlocuteurs multiples et parlant d'autres langues que le français au public. C'est un avantage certain pour une conception de l'accueil élargi au plus grand nombre, pour la mise en place de relations pacifiées, pour le plus grand bénéfice de tous.

\section{Dix ans après}

Comme on l'a vu, la bibliothèque a affiné ses modalités de fonctionnement et ses critères de recrutement. Des ajustements ont été effectués, qu'il s'agisse du nombre d'étudiants et des profils recherchés. Sans nul doute, personne ne songe à contester la présence de ce personnel. On en veut pour preuve des pots de départ des étudiants, signe incontestable de leur intégration au sein des équipes. La médiathèque José Cabanis va fêter ses 10 ans cette année, et l'on peut dire que si elle a réussi à rester un établissement très fréquenté (70 000 inscrits) et apprécié de son public, c'est grâce bien sûr à la qualité de ses services et de son offre, à la compétence de son personnel qui a su évoluer avec le temps, mais aussi grâce à ces étudiants du week-end ${ }^{4}$.

4. De mai 2004 à mai 2014, 389 étudiants ont occupé ce type de poste. 
OUVRIR PLUS ET MIEUX DANS UN CONTEXTE CONTRAINT : Monique Joly À L'INSA DE LYON, L'INNOVATION PÉDAGOGIQUE COMME MOTEUR D'UN ACCUEIL ÉLARGI

En 2009, l'ouverture de la Bibliothèque Marie Curie initie une remarquable évolution de la perception de la documentation par la communauté éducative, les chercheurs et les étudiants. Attrait du lieu et nouveaux services (mise en valeur des collections, services facilitant le travail sur place et à distance, animations culturelles et scientifiques...) emportent l'adhésion de tous, se traduisant aussitôt par une augmentation du nombre des lecteurs actifs et par une fréquentation pouvant dépasser, actuellement, 2000 lecteurs par jour.

L'augmentation des heures d'ouverture participe de cette satisfaction. Mais de quelle augmentation parle-t-on ? Alors que de considérables contraintes surviennent (diminution du nombre des emplois et des budgets) qui mettent à mal le projet longuement caressé d'accompagner l'ouverture de la bibliothèque d'une substantielle augmentation des heures d'ouverture, l'amplitude annuelle n'a pas varié en heures et a baissé en semaines ainsi qu'en jours d'ouverture annuelle comme le montrent les indicateurs ci-dessous.

QUELLE AUGMENTATION DES HEURES

TABLEAU D'OUVERTURE ? QUELQUES INDICATEURS

\begin{tabular}{|c|c|c|c|}
\hline Quelques indicateurs & 2007 & 2013 & \\
\hline Personnels en ETP (permanents et vacataires) & 23,5 & 21,5 & (2) \\
\hline Ouverture en semaines/an & 45 & 41 & (2) \\
\hline Ouverture en jours/an & 270 & 228 & 8 \\
\hline Ouverture en heures/an & 2013 & 2111 & (:) \\
\hline Ouverture en heures /semaine & 53 & 53 à 73 & (2) \\
\hline Fréquentation & $\mathrm{nc}$ & 191000 & () \\
\hline Lecteurs actifs & 3543 & 4336 & (3) \\
\hline
\end{tabular}


Quelle avancée déterminante est à l'origine, malgré tout, de la satisfaction grandissante de notre public ?

\section{DU SOUHAIT DES UTILISATEURS AU SOUHAIT DES TUTELLES}

En 2005, nos utilisateurs renouvellent leur soutien au projet de construction de la bibliothèque et montrent aux administrateurs de l'établissement combien ils attendent cet équipement, pour son accès plus direct aux collections, pour ses espaces de travail plus diversifiés et pour sa localisation plus centrale. Dans leurs remarques, les étudiants se positionnent pour un élargissement des heures d'ouverture et ils seront nombreux à le formuler dans les cahiers de suggestion.

En 2008, le SCD s'interroge sur les publics non-utilisateurs de la bibliothèque et les raisons qui motivent la non-fréquentation. Parmi les raisons exprimées, celle de leur agenda très chargé émerge : les étudiants n'ont pas le temps de venir, en journée, à la bibliothèque.

Très occupé à faire advenir la bibliothèque avec le formidable changement que va induire la valorisation, en libre accès, des imprimés en fusionnant deux bibliothèques, l'établissement inscrit dans son contrat 2007-2010 la priorité donnée au chantier de construction, avec tous ses corollaires, en laissant de côté la question des heures d'ouverture.

\section{DU SOUHAIT DES TUTELLES AU PROJET POLITIQUE}

Les années 2008 et 2009 sont des années charnière avec la parution du rapport de l'IGB ${ }^{1}$ sur l'extension des horaires d'ouverture, le positionnement politique du Président de la République et du Conseil national de l'enseignement supérieur et de la recherche (CNESER), l'expression des étudiants qui manifestent leur mécontentement, l'emploi et la rémunération des étudiants qui devient un enjeu de société. C'est ainsi que la question des horaires d'ouverture des bibliothèques s'impose dans les établissements (pour mieux étudier, pour mieux financer ses études...).

1. Georges Perrin, Améliorer l'accueil dans les bibliothèques - Propositions pour une extension des horaires d'ouverture. Rapport n²008-001 de l'Inspection générale des bibliothèques, avril 2008. 
$\mathrm{Au}$ milieu de toute cette agitation, l'INSA de Lyon se trouve dans un calendrier idéal, en 2009 pour affiner la définition des services de la nouvelle bibliothèque. Un groupe de travail issu du Conseil de la documentation, des élus dans les conseils statutaires, d'étudiants, d'élus des personnels et de la Direction générale des services (DGS), va se prononcer sur la mise en place, ou non, d'un certain nombre de services puis converger sur plusieurs scénarios d'ouverture tenant compte de la délivrance de tout ou partie des services retenus.

\section{DU PROJET POLITIQUE AU RÉALISME POLITIQUE}

Les ambitions sont grandes : 4 scénarios sont étudiés, permettant de passer de 53 à 81,5 heures/semaine avec une ouverture annuelle passant de 2154 à 3640 heures. Les horaires vont de 8 h 30 jusqu'à 23 h sur les 6 jours de la semaine.

Les ambitions sont grandes aussi pour le projet pédagogique : les scénarios prévoient une ouverture selon une amplitude hebdomadaire égale sur l'ensemble des 35 semaines de l'année consacrées aux études. Alors qu'il est de " notoriété publique » que les étudiants ont des habitudes de travail saisonnières avec d'importants pics d'activité lors de la préparation des examens de fin de semestre, le projet pédagogique retenu pour la bibliothèque vise à les inciter à produire des efforts réguliers tout au long de l'année.

Les personnels sont questionnés pour connaître leur positionnement sur ces extensions : ils sont très majoritairement peu favorables pour leur implication dans les ouvertures en soirée jusqu'à $23 \mathrm{~h}$ (pour des raisons d'organisation personnelle : absence de transports en commun et difficulté pour faire assurer la garde de leurs enfants) et un peu moins réticents en ce qui concerne le samedi (jour déjà régulièrement travaillé le matin).

C'est finalement un projet d'ouverture à 65 heures par semaine et 2665 heures annuelles qui est retenu par le Conseil de la documentation et les conseils statutaires, avec une ouverture en nocturne jusqu'à $22 \mathrm{~h}, 3$ jours par semaine. Concertation est initiée avec l'université Lyon 1 située sur le campus pour se coordonner et ne pas retenir les mêmes soirées. Pour ce qui est des moyens nécessaires, 17 vacataires doivent être recrutés pour assurer plus de 5000 heures annuelles pour un coût total d'environ 150k€ par an. 
Décision est entérinée, après concertation avec la DGS de faire assurer ces extensions d'ouverture par les seuls étudiants vacataires, puisque, nouvelle loi sur l'emploi étudiant aidant, les vacataires deviennent des personnels signant un contrat en bonne et due forme pouvant leur conférer des responsabilités comme celle d'assurer le plein pilotage de la fermeture du bâtiment, avec l'assurance d'un soutien, si nécessaire, du service sécurité de l'établissement situé à immédiate proximité.

Beau projet politique donc, teinté de réalisme parce qu'issu d'une concertation fructueuse avec toutes les parties, mais qui ne verra pas le jour! Budgéter cette dépense semble prématuré alors que beaucoup d'espoir est mis sur les économies de fonctionnement générées par la nouvelle bibliothèque qui voit ses moyens diminuer d'année en année (emplois, budget documentaire, budget de fonctionnement).

\section{DU RÉALISME POLITIQUE AU SERVICE À L'UTILISATEUR}

Dans ces conditions, la bibliothèque ouvre sans rien changer à l'amplitude horaire déjà connue des étudiants mais ses personnels doivent assumer des charges nouvelles : pour des raisons de sécurité (des personnes, des collections) l'ouverture est nécessaire avec 4 personnes, ce qui génère une augmentation du temps passé à l'accueil. De plus, la mise en place des services nouveaux, notamment numériques (accès réseaux, wifi, refonte du portail documentaire...) va porter la charge d'activité à des sommets pendant les deux années qui suivent l'emménagement dans la bibliothèque Marie Curie.

Le service à l'utilisateur augmente mais pas les horaires d'ouverture qui vont même baisser pendant cette période avec une réduction pendant les vacances scolaires (passant ainsi de 10 à 6 semaines ouvertes).

\section{DU SERVICE À L'UTILISATEUR COMME ÉVIDENCE}

Et pendant ce temps, l'établissement, impuissant devant la dégradation de ses ressources financières sera dans l'incapacité de répondre à cette demande des lecteurs. 
Mais il soutient toujours ce projet et accepte de le porter auprès du conseil régional qui encourage cette action en votant un budget d'accompagnement, en 2012, avec la perspective de renouveler cette attribution pour les quatre ans du contrat.

Le projet est retaillé afin de se rapprocher des habitudes de travail des étudiants : les horaires d'ouverture sont étendus en soirée et le samedi après-midi aux seules périodes de révision de fin de semestre.

L'ambition pédagogique n'y trouve qu'en partie son compte, mais le projet voit le jour.

Il démarre en 2013 et offre, 10 semaines dans l'année, une ouverture jusqu'à $22 \mathrm{~h}$ en semaine et $17 \mathrm{~h}$ le samedi. Conformément aux vœux exprimés par les personnels, ceux-ci ne sont pas sollicités pour cette extension des horaires ; ce sont des étudiants vacataires de bon niveau (masters 1 et 2 , pour la plupart issus des filières bibliothèques et documentation) qui sont recrutés.

Les étudiants plébiscitent cette offre et la fréquentation quotidienne double pendant cette période et $22 \%$ de la population potentielle répond à une enquête de satisfaction : ils sont $85 \%$ à considérer que ce service leur permet de mieux préparer les examens.

Succès incontestable donc!

\section{DU SERVICE À L'UTILISATEUR COMME MOTEUR DE L'INNOVATION PÉDAGOGIQUE}

Plus intéressant, $28 \%$ souhaitent que ces horaires deviennent les horaires classiques de la bibliothèque et $28 \%$ souhaitent que la bibliothèque ouvre encore plus tard le soir.

En effet, les étudiants voient de plus en plus l'intérêt de cette nouvelle bibliothèque pour conduire leurs projets de groupe : des salles dédiées existent, la documentation peut être consultée sur place, tout ceci représentant des conditions idéales d'études.

Et d'une certaine façon leur demande d'une généralisation des heures d'ouverture à l'ensemble de l'année montre qu'ils s'enthousiasment pour les innovations pédagogiques, ce qui ne peut qu'encourager l'établissement à s'en saisir plus encore. 


\section{DE L'INNOVATION PÉDAGOGIQUE COMME MOTEUR DE L'EXTENSION DES HORAIRES D' OUVERTURE ?}

Enfin, perspective passionnante programmée dans un futur proche, un projet national réunissant plus de 1000 étudiants dispersés sur l'ensemble du territoire, va en réunir une centaine dans la bibliothèque pour un marathon consacré à la créativité et à l'innovation industrielle, pendant 3 jours non-stop 2 .

Quelle belle mise en contexte pour illustrer comment la bibliothèque peut être soutenue par les acteurs des études (les enseignants et les étudiants) pour une offre de services qui permette l'avènement de nouvelles façons d'enseigner et d'utiliser les bibliothèques. 


\section{MÉ MENTO par Georges Perrin}

\section{UNE TRĖS GRANDE VARIÉTÉ DE PRATIQUES : DES PISTES POUR RÉUSSIR}

Comme le montrent assez bien les témoignages présentés dans les contributions précédentes, il n'y a ni recette ni pratique unique pour prévoir ou élargir les horaires d'ouverture d'une bibliothèque ou d'un réseau. Tout est tributaire du contexte local : les rythmes de vie des populations, l'environnement urbain et la localisation des espaces de bibliothèque, la dimension même de la bibliothèque, l'existence ou non d'un réseau, la politique culturelle développée par la municipalité et le degré de priorité qu'elle y accorde, l'organisation et les pratiques administratives, notamment la politique de gestion des personnels de la collectivité, sans compter le facteur non négligeable du niveau de conviction des personnes ayant initié le projet et/ou ayant la charge de le mettre en œuvre. Bref, de nombreux facteurs s'entremêlent, et les conditions de la réussite consistent à mettre en phase, dans toute la mesure du possible, cette diversité, à réduire les éventuelles contradictions si minimes soient-elles et à atteindre l'objectif fixé, avec la perspective d'améliorations ultérieures si nécessaire.

Malgré tout cela, il est possible de dégager de l'ensemble des cas exposés et des réflexions et conseils émis dans ces pages, quelques pistes assez générales qui devraient éviter quelques erreurs et aider à l'orientation des projets dans ce domaine.

On peut les énoncer par rapport aux responsabilités et rôles de chacune des catégories de responsables de la mise en œuvre du projet, à savoir les décideurs " politiques » et les professionnels en charge de la bibliothèque et de son fonctionnement. 


\section{POUR LES PROFESSIONNELS}

\section{Faire de l'accueil des publics la mission prioritaire de la bibliothèque}

- Renouveler la conception du métier et placer la valorisation des services aux publics au centre de l'activité ;

- passer de la simple gestion de collections à la médiation active de leurs contenus auprès du public ;

- dans cette perspective, reconsidérer de façon fondamentale l'équilibre et la répartition des moyens et du temps de travail entre la gestion technique et administrative interne et l'accueil des publics. Faire coïncider les deux dans toute la mesure du possible (Condé-sur-Noireau) ;

- automatiser autant que faire se peut la gestion des prêts et retours (automates de prêt, boîtes de retour extérieures) ;

- externaliser, dans toute la mesure du possible, toutes les tâches matérielles : équipement, reliure.

\section{Dialoguer avec son public pour mieux le connaître}

- Mettre en œuvre les méthodes d'analyse nécessaires à la connaissance des divers publics fréquentant la bibliothèque et de leurs attentes et de leurs dispositions réelles. Procéder par enquêtes ou questionnaires portant sur les attentes des usagers sur leurs comportements dans les espaces de la bibliothèque et sur leurs disponibilités temporelles réelles ;

- associer des représentants des usagers à la réflexion préparatoire, dans un souci de dialogue concernant le fonctionnement de la bibliothèque, et prendre en compte, chaque fois que ceci apparaît possible, leurs souhaits d'améliorations diverses des services qui leur sont proposés. 


\section{Réorganiser et mutualiser le travail interne}

- Mutualiser avec d'autres bibliothèques et concentrer en un minimum de lieux le traitement intellectuel et matériel des documents. Ceci est valable à l'échelle des réseaux communaux ou intercommunaux. On peut penser (rêver ?) qu'une telle mutualisation pourrait se mettre en place à une échelle plus vaste, au niveau d'un département ou d'une région, par exemple, comme ceci se pratique dans les pays d'Europe du Nord depuis plus de 30 ans ;

- développer une politique de polyvalence des tâches pour chaque personne au sein du service, en évitant une trop grande spécialisation individuelle, de sorte que tous les personnels soient appelés à participer à l'accueil du public. Ceci est à déterminer dans le respect des statuts, et en tenant compte du niveau de responsabilité de chaque catégorie d'agents.

\section{se former en équipe}

- Pour que tout un chacun soit convaincu du bien-fondé de la priorité accordée au public, mais aussi et surtout afin de pouvoir mettre en œuvre avec succès des méthodes susceptibles d'améliorer l'accueil du public, il est indispensable de prévoir une formation adéquate pour l'ensemble des personnels de la bibliothèque, et non pas pour les seuls préposés à l'accueil du public ;

- des visites ou stages au sein de bibliothèques ayant déjà mis en œuvre des ouvertures élargies peuvent se révéler des plus utiles. 


\section{Associer l'ensemble de l'équipe à la préparation et à la mise en œuvre de l'élargissement de l'ouverture}

- Dans le respect des conditions du dialogue social, définir les clauses de participation des personnels avec leurs représentants : volontariat ou participation généralisée assortie de dérogations, compensations en temps ou en rémunération, etc. ;

- dans les réseaux urbains, solliciter la participation de l'ensemble des équipes pour le fonctionnement de la bibliothèque sur les nouvelles plages horaires, même si l'élargissement des ouvertures ne concerne qu'une seule bibliothèque ;

- une fois les objectifs clairement définis (nocturnes et/ou ouverture du dimanche), inviter l'ensemble des agents à prendre part à la définition des nouveaux horaires. Déterminer clairement avec eux les lieux (où), les plages de temps (quand), les services à offrir (quoi ?) pour les horaires élargis et les moyens humains et matériels complémentaires nécessaires (comment) ;

- déterminer avec l'équipe la hauteur des moyens nécessaires, notamment en personnels complémentaires pour établir avec souplesse le planning optimal ;

- définir ensemble, et selon les compétences respectives, les complémentarités entre les services offerts à distance et ceux qui sont proposés dans les locaux de la bibliothèque.

\section{POUR LES DÉCIDEURS : MAIRES, PRÉSIDENTS DE COMMUNAUTÉ D'AGGLOMÉRATION OU D'UNIVERSITÉ}

\section{Affirmer clairement la volonté politique qui s'attache au projet d'élargissement des horaires d'ouverture}

- Formuler très clairement les exigences quant à l'optimisation attendue des horaires d'ouverture ; 
- quelle que soit l'origine du projet (élus ou direction de la bibliothèque) affirmer avec force et constance la volonté politique de mettre en œuvre une politique des horaires d'ouverture adaptée aux réelles disponibilités des publics, et de conduire le projet jusqu'à sa réalisation finale ;

- assurer aux personnes qui, au sein de la bibliothèque, ont la responsabilité de cette mise en œuvre (direction et encadrement), un soutien permanent à la fois pour le respect des objectifs initiaux et les délais de réalisation ;

- veiller à la qualité du dialogue social notamment dans les relations entre les hauts responsables de l'administration municipale (DGS, DGA, DGC) et les équipes de la bibliothèque ;

- garantir, après concertation avec les responsables de la bibliothèque, l'octroi des moyens (personnels et/ou crédits) nécessaires à l'élargissement des horaires d'ouverture, en complémentarité avec les moyens d'autres services de la collectivité (sécurité, communication, entre autres).

\section{CONJOINTEMENT, POUR LES DÉCIDEURS POLITIQUES ET LES RESPONSABLES DE LA BIBLIOTHĖQUE}

- Préparer en concertation un plan de communication en direction du public pour le préparer aux nouvelles conditions d'accueil, et pour l'informer des éventuels nouveaux services proposés ;

- avant l'ouverture de nouveaux horaires, déterminer ensemble une méthode et un calendrier d'évaluations régulières du fonctionnement et du taux de satisfaction des usagers, afin d'améliorer au fil du temps, autant que faire se peut, ces horaires et les services proposés ;

- étudier localement, sur le plan politique et professionnel, toutes les complémentarités de services et d'horaires d'accueil avec 
la ou les bibliothèques d'une autre obédience, afin de proposer aux usagers les meilleures possibilités d'accueil et d'accès aux ressources documentaires locales.

L'énoncé de ces quelques pistes, pour utile qu'il soit, n'est certes pas exhaustif, et pour tout professionnel qui a l'intention d'initier un tel projet, il convient de regarder de plus près d'autres expériences peut-être plus analogues par la dimension et la composition sociale de la population à desservir et par les rythmes de vie qui en découlent, ou, dans une université, par la dominante disciplinaire concernée.

Pour conclure ce panorama de conseils il convient d'évoquer l'un des facteurs de réussite les plus déterminants, à savoir l'opportunité du projet. Tout montre que les projets les plus aboutis sont ceux nés à l'occasion de la construction, de l'agrandissement ou de la rénovation d'un bâtiment. Il s'agit là de la meilleure des chances à saisir pour la mise en place d'un accueil de qualité et, notamment d'horaires d'ouverture qui satisfassent l'attente de tous les publics sans exception. 


\section{SIGLES ET ACRONYMES}

ANTTNC

Agent non-titulaire à

temps non complet

BMVR

Bibliothèque municipale à vocation régionale

CNESER

Conseil national de l'enseignement supérieur et de la recherche

\section{CPER}

Contrat de plan État-Région

DGA

Direction générale de l'administration

DGC

Direction générale de la culture

DGS

Direction générale des services

\section{DRH}

Direction des ressources humaines

DUT

Diplôme universitaire de technologie

ECN

Examen classant national ( $6^{\mathrm{e}}$ année d'études de médecine)

ETP

Équivalent temps plein
IAT

Indemnité d'administration et de technicité

\section{IFTS}

Indemnité forfaitaire pour travaux supplémentaires

IGB

Inspection générale des bibliothèques

\section{IHTS}

Indemnité horaire pour travaux supplémentaires

INSA

Institut national des sciences appliquées

\section{PACS}

Pacte civil de solidarité

POE

Post-occupancy Evaluation, Évaluation post-occupationnelle

\section{RAP/MESR}

Rapport annuel de performance du ministère de l'Enseignement supérieur et de la Recherche

\section{RFID}

Radio frequency identification, Radio identification

RSA

Revenu de solidarité active 


\section{SHON}

Surface hors œuvre nette

SHS

Sciences humaines et sociales

SIGB

Système intégré de gestion de bibliothèque
SMIC

Salaire minimum interprofessionnel de croissance

\section{STAPS}

Sciences et techniques des activités physiques et sportives 


\section{GLOSSAIRE}

\section{Autoformation}

Cette notion, voisine de l'« autodidaxie ", désigne l'action de formation que détermine l'individu par lui-même et pour lui-même. Elle vise généralement à s'insérer professionnellement dans le cadre de la recherche d'un premier emploi, d'un retour à l'emploi ou d'un changement d'orientation professionnelle. Mais elle peut également concerner l'apprentissage d'une langue étrangère ou du code de la route. Elle peut être suivie dans un système éducatif, dans des groupes sociaux, par recours à des systèmes d'information accessibles à distance. On trouve dans de nombreuses bibliothèques de la documentation et des moyens matériels (espaces dédiés, terminaux informatiques en nombre) pour s'autoformer.

\section{Baby bust}

Nom donné à la génération qui a suivi celle des «baby boomers ». Cette génération est également nommée "Génération $\mathrm{X}$ », selon la classification des historiens américains William Strauss et Neil Hawe, auteurs d'une " théorie des générations ». Elle englobe les personnes nées entre 1960 et 1981.

\section{Back office}

Cette notion englobe tout le travail de gestion interne de la bibliothèque : gestion administrative et financière, gestion bibliothéconomique (acquisitions, catalogage), gestion logistique (espaces, mobilier, matériel, informatique, équipement des documents, etc.), gestion des ressources humaines, etc. En bref, tout ce que le public ne voit pas.

\section{Démarche qualité}

Ce terme désigne les tentatives et approches en vue d'obtenir une certification, en général un certificat ISO 9001, du nom de la norme d'amélioration de la qualité du fonctionnement d'une entreprise, d'une association ou d'un service public. Cette norme est opposable à tous types de structures, publiques ou privées. Elle préconise une démarche de progression dans la qualité de la structure concernée, portant entre autres sur sa stratégie, son organisation, ses ressources humaines, les compétences qui s'y exercent et la satisfaction de ses usagers. En règle générale, dans une commune, cette démarche qualité visant à satisfaire aux exigences de la norme ISO 9001 est imposée par le maire à l'ensemble des services municipaux, dont la bibliothèque. 


\section{Discontinuité des horaires}

De nombreuses bibliothèques pratiquent des horaires d'ouverture au public qui sont difficilement lisibles et ne correspondent pas toujours aux attentes de certaines catégories d'usagers potentiels. La fermeture de la bibliothèque certaines demi-journées en semaine, la fermeture cumulée du dimanche et du lundi, la coupure méridienne, constituent autant d'obstacles à un accès " égalitaire " à la bibliothèque en privant d'accès les personnes qui ne sont pas disponibles aux heures d'ouverture.

\section{Évaluation post-occupationnelle}

Il s'agit d'une méthode d'évaluation qualitative utilisée aujourd'hui par les bureaux d'études spécialisés. Elle procède par entretiens et focus-groupes auprès des usagers sur les choix souhaités pour la conception d'une nouvelle bibliothèque.

\section{Fréquentants}

Le public qui accède aujourd'hui aux bibliothèques n'a plus forcément le désir de s'inscrire et d'emprunter des documents. En effet, une part grandissante des usagers fréquente la bibliothèque en tant que lieu public où tout le monde est accueilli sans stigmatisation et où ils savent pouvoir trouver gratuitement toutes sortes d'informations et rencontrer des gens qui leur ressemblent ou qui leur sont très différents. Dans toutes les enquêtes sur les publics des bibliothèques, ils sont désormais désignés sous le terme de «fréquentants».

\section{Front office}

Par opposition au back office, le front office concerne tout le travail et l'offre au contact du public : accueil et renseignements, gestion des prêts-retours, mise à disposition des terminaux informatiques, actions culturelles, et plus généralement, l'ensemble des services proposés aux usagers, dans la bibliothèque et à distance.

\section{Génération $\mathrm{X}$}

Voir plus haut : Baby bust.

\section{Génération Y}

Selon William Strauss et Neil Hawe, la génération $\mathrm{Y}$ comprend toutes les personnes nées entre 1982 et 2004, dénommées également digital natives ou net generation. Cette génération est contemporaine du lancement de l'ordinateur individuel, des jeux video et du téléphone portable, toutes technologies qu'elle utilise massivement.

\section{Génération Z}

Selon la même catégorisation, cette génération, née à partir de 1995, a toujours connu les technologies de l'information. De ce fait, elle est hyper-connectée. 
Aussi, dans ses modes de vie, elle tend à abolir toute notion de temps (actifs et joignables à tout moment) et d'espace (proximité et distance se confondent dans un même processus d'accès).

\section{Harmonisation des horaires}

La combinaison harmonieuse des horaires d'ouverture de la bibliothèque doit répondre à une double nécessité : d'une part, celle d'offrir aux usagers une possibilité d'accès la plus large et continue possible, d'autre part, de rendre compatibles ce large accès et les contraintes personnelles des agents (vie familiale, transports, et contraintes diverses).

\section{Learning Centre}

Littéralement " centre d'apprentissage », le Learning Centre est né aux États-Unis et en Grande-Bretagne dans les années 1990. Développé d'abord dans les universités, il s'identifie soit comme une bibliothèque entière, soit comme un secteur de celle-ci. Le concept se définit essentiellement par l'imbrication de la ressource documentaire et de la pédagogie. Un accent particulier est mis sur l'assistance à l'usager et le travail en groupe. Né dans les universités, le modèle essaime désormais dans les grandes bibliothèques municipales.

\section{Médiation ambulatoire}

Nouvelle définition de la relation des personnels au public pour le fonctionnement du service. De nombreux services publics (voir la Poste) mettent aujourd'hui en œuvre cette nouvelle manière d'accueillir et de prendre en charge le public en demandant aux personnels d'être présents au milieu de ce dernier pour répondre rapidement et avec efficacité à ses demandes.

\section{NoctamBU}

Ce label est attribué aux bibliothèques des universités qui ouvrent plus de 65 heures par semaine, notamment en pratiquant des ouvertures en nocturne. L'attribution de ce label ouvre droit à une subvention permettant de prendre en charge les vacations nécessaires à ces ouvertures supplémentaires. Aujourd'hui, 42 bibliothèques universitaires bénéficient de ce label.

\section{Ouverture}

Dans l'évolution en cours des bibliothèques, la notion d'ouverture doit s'entendre dans plusieurs sens : celui, bien sûr, de l'accès de toutes les catégories de public grâce à des horaires d'accueil suffisants, mais aussi celui d'un élargissement des services qu'elle doit offrir à ce public, sur place et à distance. Cette ouverture correspond à un renouvellement réfléchi non seulement des horaires d'ouverture, mais 
également de l'utilisation des espaces, de leur finalité et de leur qualité. Cet esprit d'ouverture doit aussi remettre en valeur le rôle social, autant que culturel, de la bibliothèque.

\section{Post-0ccupancy Evaluation (POE)}

Voir Évaluation post-occupationnelle.

\section{Progrès qualitatif}

L'augmentation des horaires d'ouverture a pour objectif d'obtenir un progrès quantitatif quant au nombre d'usagers nouveaux susceptibles de fréquenter la bibliothèque. Mais ce même élargissement de l'ouverture peut constituer pour les habitués un progrès qualitatif, dans la mesure où elle leur offre un meilleur confort d'accès, la possibilité de venir le dimanche seul ou en groupe (famille, amis), l'accroissement du profit intellectuel et personnel qu'ils retirent de la fréquentation des collections, etc.

\section{Séjourneurs}

Dans toutes les bibliothèques, on constate aujourd'hui qu'une proportion non négligeable du public ne vient pas pour emprunter des documents, mais pour utiliser les services mis à sa disposition. Certains usagers passent une partie importante de leur temps sur place, pour travailler, lire ou faire des recherches concernant la vie pratique (recherche d'emploi, santé, orientation, apprentissage des langues, etc.). Ces personnes, qui sont des habitués des bibliothèques (retraités, chômeurs ou autres) sont appelées par les observateurs, les « séjourneurs ».

\section{Temporalité urbaine}

Il s'agit essentiellement des rythmes d'activité perceptibles dans une ville, grande ou petite, en fonction des services proposés (commerces, écoles, collèges et lycées, transports et services de toutes natures) et des modes de vie (horaires de travail, horaires scolaires, horaires de loisirs, vie personnelle et familiale, etc.) des différentes catégories de population. Cette temporalité, autrefois ordonnée autour du travail, a beaucoup évolué au cours des dernières décennies sous l'influence des nouvelles technologies de l'information et de l'évolution de la mobilité due elle-même à l'évolution de l'habitat (plus ou moins éloigné des centres de l'activité réelle : travail, services, loisirs) et à la qualité et souplesse d'utilisation des transports.

\section{Usager concret}

Contrairement à l'individu abstrait, objet de tous les soins d'une bibliothèque universaliste proposant des collections et des services susceptibles de développer le potentiel culturel de l'ensemble des citoyens, l'usager mise aujourd'hui davantage sur sa singularité. Il entend se définir lui-même, décider par lui-même et pour lui-même de ses choix. Aussi aborde-t-il les services de la biblio- 
thèque avec sa sensibilité personnelle et non selon les codes fixés jusqu'ici de façon théorique et universaliste. Tel est l'usager concret dont les aspirations et le comportement obligent à redéfinir le profil et les tâches des personnels des bibliothèques.

\section{Usager potentiel}

On déplore depuis plusieurs années une baisse d'intérêt du public pour les bibliothèques. Ce constat repose sur le strict examen du nombre des "lecteurs " inscrits. Il ne prend pas en compte la totalité des «fréquentants » qui permet de penser que le public potentiel des bibliothèques peut encore croître. Les nouvelles pratiques horaires mises en œuvre dans certaines bibliothèques (ouverture du dimanche, nocturne), ainsi que l'introduction de nouveaux services concernant la vie quotidienne (autoformation en langues, aide à la recherche d'emploi, informations sur la santé, etc.) suscitent l'arrivée en bibliothèque de publics nouveaux et plus variés. C'est ce « bassin » d'usagers potentiels qu'il convient aujourd'hui d'explorer pour continuer à augmenter la diversité du public des bibliothèques. 


\section{BIBLIOGRAPHIE}

\section{OUVRIR PLUS : POURQUDI ?}

Dominique Arot, L'extension des horaires d'ouverture des bibliothèques : progrès et obstacles, Rapport $n^{\circ} 2012-005$ de l'Inspection générale des bibliothèques, novembre 2012. [En ligne] : <http://cache.media. enseignementsup-recherche.gouv.fr/ file/2012/35/3/horairesouverture_rapport_definitif_236353.pdf >.

Marie Déage, Ouvrir plus, ouvrir mieux, ouvrir autrement? Faisabilité et pertinence des extensions des horaires d'ouverture en bibliothèques universitaires, Mémoire d'étude de conservateur des bibliothèques, sous la direction d'Élisabeth Noël : Villeurbanne, enssib, 2010. [En ligne] : < http://www. enssib.fr/bibliotheque-numerique/ documents/48193-ouvrir-plus-ouvrirmieux-ouvrir-autrement-faisabiliteet-pertinence-des-extensions-des-horaires-d-ouverture-en-bibliothequesuniversitaires $>$.

Jean-François Jacques, « Les horaires d'ouverture des bibliothèques : un service public de la culture ", Bibliothèque(s), n 53/54, 2010, pp. 33-37. [En ligne] : < http://www. enssib.fr/bibliotheque-numerique/ documents/59925-53-54-service-public.pdf\#page $=35>$.

Georges Perrin, "Accueillir de nouveaux publics? Oui ! Mais quand?», Bulletin des bibliothèques de France, 2009, n 5, pp. 24-27. [En ligne] : < http://bbf.enssib.fr/consulter/bbf2009-05-0024-004 >.

Georges Perrin, Améliorer l'accueil dans les bibliothèques. Propositions pour une extension des horaires d'ouverture, Rapport $n^{\circ} 2008-001$ de l'Inspection générale des bibliothèques, avril 2008. [En ligne] : < http://media. enseignementsup-recherche.gouv.fr/ file/Rapports/26/9/ameliorer_l_accueil_dans_les_bibliotheques_rapport_2008-001_26269.pdf >.

\section{LE TEMPS DE LA VILLE}

Jean-Yves Boulin, « Les temps de la ville », Revue Projet, mars 2003, $\mathrm{n}^{\circ}$ 273. [En ligne] : < http://www. revue-projet.com/articles/2003-1-lestemps-de-la-ville/ >.

François Ascher, < Du vivre en juste à temps au chrono-urbanisme $>$, Les annales de la recherche urbaine, 1997, nº 77 [En ligne] : < http://www. 
annalesdelarechercheurbaine.fr/IMG/ pdf/Ascher_ARU_77.pdf >.

Bruno Marzloff, « Le temps collaboratif ", Place publique, site des initiatives citoyennes, 2008. [En ligne] : $<$ www.place-publique.fr/spip.php?pa ge=recherche\&recherche $=$ le + temps $+c$ ollaboratif >.

Association Tempo territorial : $<$ http://tempoterritorial.free.fr >.

\section{CONNAÎTRE SON PUBLIC}

$+++++++++++++++++++++++++++++++$

Christophe Evans (dir.), Mener l'enquête. Guide des études de public en bibliothèque, Villeurbanne, Presses de l'enssib, 2011 (coll. La Boîte à outils ; 22).

Plein sens/MOTif, « Happy hours. Évaluation de l'impact des horaires d'ouverture sur la fréquentation et les usages des publics en bibliothèque publique », Rapport d'étude, 2011. [En ligne] : < http://www.lemotif.fr/fichier/ motif_fichier/277/fichier_fichier_ etude.happy.hoursnouveau.pdf >.

\section{LE PUBLIC : ATTENTES ET ATTITUDES}

Agnès Camus, Jean-Michel Cretin, Christophe Evans, Les habitués. Le microcosme d'une grande biblio- thèque, Paris, Bibliothèque publique d'information, 2000 (coll. Études et recherche).

Serge Paugam, Camila Giorgetti, Des pauvres à la bibliothèque : enquête au Centre Pompidou, Paris, Presses universitaires de France, 2013 (coll. Le lien social).

Claude Poissenot, «L'irruption de l'usager concret. Du "service public" aux "services aux publics" », Bibliothèque(s), n 53/54, 2010, pp. 24-27. [En ligne] : < http://www. enssib.fr/bibliotheque-numerique/ documents/59925-53-54-service-public.pdf\#page $=26$ >.

\section{UNE NOUVELLE GÉNÉRATION D'USAGERS}

Claudine Attias-Donfut, Sociologie des générations : l'empreinte du temps, Paris, Presses universitaires de France, 1998 (coll. Le sociologue).

Michel Barabel, Olivier Meier, André Perret, Travailler avec les nouvelles générations $Y$ et $Z$, Paris, StudyramaPro, 2012 (coll. Focus RH).

Julien Pouget, Intégrer et manager la génération $Y, 2^{\mathrm{e}}$ éd., Paris, Vuibert, 2013 (coll. Lire Agir. Ressources humaines).

Olivier Rollot, La génération Y, Paris, Presses universitaires de France, 2012. 
DES PROTOTYPES INTÉRESSANTS

$++++++++++++++++++++++++++++++$

Suzanne Jouguelet, Les Learning centres : un modèle international de bibliothèque intégrée à l'enseignement et à la recherche, Rapport $n^{\circ}$ 2009-022 de l'Inspection générale des bibliothèques, décembre 2009. [En ligne] : < http://cache.media. enseignementsup-recherche.gouv.fr/ file/2009/33/6/Rapport_Learning_ Centers_7-12_RV_131336.pdf >.

Ophélie Ramonatxo, Organisation des services publics à Drancy: pratiques d'hier à aujourd'hui, 2009, [En ligne] : < http://www.lebibwebzine.blogspot. fr/2009/10/conjuguer-accueil-du-public-et_14.html >.

\section{RÉUSSIR LES NÉGOCIATIONS AVEC LES ÉQUIPES}

Association des directeurs et personnels de direction des bibliothèques universitaires et de la documentation (ADBU), Guide sur l'extension des horaires d'ouverture, 2011. [En ligne] : < www.adbu.fr/actualites/371/ >.

Jean-François Jacques, « Organisation, rémunérations : propositions méthodologiques », Tempo territorial, 2011. [En ligne] : < http://tempoterritorial.free.fr/IMG/pdf/biblio-web. pdf >. 


\section{LISTE DES ILLUSTRATIONS}

Figure. Les mots de l'ouverture : nuage de mots à partir des commentaires de la pétition « Ouvrons plus les bibliothèques »

Graphique. Présence des usagers à la BU selon les heures

Graphique. Occupation des espaces de travail de la BU

Encadré. Rythmes de vie :

les grandes évolutions en chiffres

Schéma. Les impacts de la réforme scolaire

Encadré. Catégories de population/ horaires de fréquentation : enquête 2011

Encadré. L'amplitude horaire des BM en France : quelques chiffres
Encadré. L'amplitude horaire des BU en France : quelques chiffres

Encadré. Rémunération du travail dominical

Tableau. Quelques chiffres sur la BU Santé (2012)

Tableau. Les horaires NoctamBU (sauf les jours fériés)

Encadré. Les missions des tuteurs

Encadré. Les missions des agents de sécurité

Tableau. Quelle augmentation des heures d'ouverture? Quelques indicateurs 


\section{LISTE DES AUTEURS}

Chrystelle Amblard

Responsable de la mission Temps et territoire, Montpellier agglomération

\section{Pierre-Yves Cachard}

Directeur du Service commun de la documentation de l'université du Havre

\section{Jean Chaguiboff}

Fondateur et ancien directeur de

l'Agence « Plein Sens »

\section{Héloïse Courty}

Directrice de la Médiathèque L'Écho, Le Kremlin-Bicêtre

\section{Pierre Franqueville}

Directeur de l'Agence d'ingénierie culturelle et artistique $\mathrm{ABCD}$ (Paris)

\section{Hélène Grognet}

Directrice du Service commun de la documentation de l'université de Nantes

\section{Charlotte Henard}

Responsable des pôles Sociétés et Actualité à la Médiathèque José Cabanis (Toulouse)

\section{Jean-François Jacques}

Conservateur en chef honoraire des bibliothèques
Monique Joly

Directrice, SCD Doc'INSA, Service commun de la documentation de l'INSA de Lyon

\section{Emmanuelle Paulet-Grandguillot}

Responsable de la Bibliothèque universitaire Santé, du Service commun de la documentation de l'université de Nantes

\section{Georges Perrin}

Inspecteur général honoraire des bibliothèques

\section{Claude Poissenot}

Enseignant-chercheur à l'IUT NancyCharlemagne, Centre de recherche sur les médiations (CREM) de l'université de Lorraine

\section{Ophélie Ramonatxo}

Directrice de la Médiathèque de l'Institut français du Royaume-Uni (Londres)

\section{Isabelle Vazard}

Responsable de la Médiathèque de Condé-sur-Noireau

\section{Isabelle Westeel}

Directrice du Service commun de la documentation de l'université Lille 3 
Secrétariat d'édition:

Silvia Ceccani

Mise en page:

Cédric Vigneault

Conception graphique:

atelier Perluette, 69001 Lyon.

< http://www.perluette-atelier.com >

Dépôt légal: $2^{\mathrm{e}}$ semestre 2014 


\section{LA BOÎTE À OUTILS}

Les manuels de cette collection visent à fournir aux professionnels des ouvrages pratiques pour conduire des projets bibliothéconomiques d'actualité concernant aussi bien des bibliothèques publiques que des bibliothèques universitaires ou de recherche. Écrit à plusieurs mains, chaque volume est coordonné par un professionnel des bibliothèques.

Catherine Jackson directrice de la collection

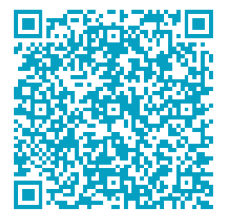

$++++++++++++++++++++++$

\section{PRESSES DE L'enssib}

École nationale supérieure des sciences de l'information et des bibliothèques

17-21 boulevard du 11 novembre 1918 69623 Villeurbanne Cedex

Tél. 0472444343

Fax 0472444344

< http://www.enssib.fr/presses >
Dans la même collection

BA0 \#23 (2011)

Créer des services innovants. Stratégies et répertoire d'actions pour les bibliothèques sous la direction de Marie-Christine Jacquinet

BA0 \#24 (2011)

Mener un projet international: bibliothèques françaises et coopération internationale sous la direction de Raphaëlle Bats

BA0 \#25 (2012)

Développer la médiation documentaire numérique sous la direction de Xavier Galaup

BA0 \#26 (2012)

Apprendre à gérer des collections patrimoniales en bibliothèque

sous la direction de Dominique Coq

BA0 \#27 (2012)

Faire connaître et valoriser sa bibliothèque : communiquer avec les publics sous la direction de Jean-Marc Vidal

BA0 \#28 (2013)

Favoriser l'insertion professionnelle et l'accès à l'emploi: les atouts des bibliothèques sous la direction de Georges Perrin

BA0 \#29 (2014)

Intégrer des ressources numériques dans les collections

sous la direction de Géraldine Barron et Pauline Le Goff-Janton

BA0 \#30 (2014)

Produire des contenus documentaires en ligne: quelles stratégies pour les bibliothèques ? Sous la direction de Christelle di Pietro

À paraître

$++++++++++++++++++++++++++++++++++$

BA0 \#32 (2015)

Conduire et accompagner le changement en bibliothèque : gérer les personnels sous la direction de Christophe Pérales 